\title{
Azimuthal single- and double-spin asymmetries in semi-inclusive deep-inelastic lepton scattering by transversely polarized protons
}

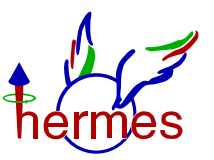

\section{The HERMES Collaboration}
A. Airapetian, ${ }^{13,16}$ N. Akopov, ${ }^{26}$ Z. Akopov, ${ }^{6}$ E.C. Aschenauer, ${ }^{7}$ W. Augustyniak, ${ }^{25}$
R. Avakian, ${ }^{26, a}$ A. Bacchetta, ${ }^{21}$ S. Belostotski, ${ }^{19, a}$ V. Bryzgalov, ${ }^{20}$ G.P. Capitani, ${ }^{11}$
E. Cisbani, ${ }^{22}$ G. Ciullo, ${ }^{10}$ M. Contalbrigo, ${ }^{10}$ W. Deconinck, ${ }^{6}$ R. De Leo, ${ }^{2}$
E. De Sanctis, ${ }^{11}$ M. Diefenthaler, ${ }^{9}$ P. Di Nezza, ${ }^{11}$ M. Düren, ${ }^{13}$ G. Elbakian, ${ }^{26}$
F. Ellinghaus, ${ }^{5}$ A. Fantoni, ${ }^{11}$ L. Felawka, ${ }^{23}$ G. Gavrilov, ${ }^{6,19,23}$ V. Gharibyan, ${ }^{26}$
D. Hasch, ${ }^{11}$ Y. Holler, ${ }^{6}$ A. Ivanilov, ${ }^{20}$ H.E. Jackson, ${ }^{1, a}$ S. Joosten, ${ }^{12}$ R. Kaiser, ${ }^{14}$
G. Karyan, ${ }^{6,26}$ E. Kinney, ${ }^{5}$ A. Kisselev, ${ }^{19}$ V. Kozlov, ${ }^{17}$ P. Kravchenko, ${ }^{9,19}$ L. Lagamba, ${ }^{2}$
L. Lapikás, ${ }^{18}$ I. Lehmann, ${ }^{14}$ P. Lenisa, ${ }^{10}$ W. Lorenzon, ${ }^{16}$ S.I. Manaenkov, ${ }^{19}$
B. Marianski, ${ }^{25, a}$ H. Marukyan, ${ }^{26}$ Y. Miyachi, ${ }^{24}$ A. Movsisyan, ${ }^{10,26}$ V. Muccifora, ${ }^{11}$
Y. Naryshkin, ${ }^{19}$ A. Nass, ${ }^{9}$ G. Nazaryan, ${ }^{26}$ W.-D. Nowak, ${ }^{7}$ L.L. Pappalardo, ${ }^{10}$
P.E. Reimer, ${ }^{1}$ A.R. Reolon, ${ }^{11}$ C. Riedl, ${ }^{7,15}$ K. Rith, ${ }^{9}$ G. Rosner, ${ }^{14}$ A. Rostomyan, ${ }^{6}$
J. Rubin, ${ }^{15}$ D. Ryckbosch, ${ }^{12}$ A. Schäfer, ${ }^{21}$ G. Schnell, ${ }^{3,4,12}$ B. Seitz, ${ }^{14}$ T.-A. Shibata, ${ }^{24}$
V. Shutov, ${ }^{8}$ M. Statera, ${ }^{10}$ A. Terkulov, ${ }^{17}$ M. Tytgat, ${ }^{12}$ Y. Van Haarlem, ${ }^{12}$
C. Van Hulse, ${ }^{12}$ D. Veretennikov, ${ }^{3,19}$ I. Vilardi, ${ }^{2}$ S. Yaschenko, ${ }^{9}$ D. Zeiler, ${ }^{9}$
B. Zihlmann ${ }^{6}$ and P. Zupranski ${ }^{25}$

\footnotetext{
${ }^{1}$ Physics Division, Argonne National Laboratory, Argonne, Illinois 60439-4843, U.S.A.

${ }^{2}$ Istituto Nazionale di Fisica Nucleare, Sezione di Bari, 70124 Bari, Italy

${ }^{3}$ Department of Theoretical Physics, University of the Basque Country UPV/EHU, 48080 Bilbao, Spain

${ }^{4}$ IKERBASQUE, Basque Foundation for Science, 48013 Bilbao, Spain

${ }^{5}$ Nuclear Physics Laboratory, University of Colorado, Boulder, Colorado 80309-0390, U.S.A.

${ }^{6}$ DESY, 22603 Hamburg, Germany

${ }^{7}$ DESY, 15738 Zeuthen, Germany

${ }^{8}$ Joint Institute for Nuclear Research, 141980 Dubna, Russia
}

\footnotetext{
${ }^{\mathrm{a}}$ Deceased.
} 
${ }^{9}$ Physikalisches Institut, Universität Erlangen-Nürnberg, 91058 Erlangen, Germany

${ }^{10}$ Istituto Nazionale di Fisica Nucleare, Sezione di Ferrara, and Dipartimento di Fisica e Scienze della Terra, Università di Ferrara, 44122 Ferrara, Italy

${ }^{11}$ Istituto Nazionale di Fisica Nucleare, Laboratori Nazionali di Frascati, 00044 Frascati, Italy

${ }^{12}$ Department of Physics and Astronomy, Ghent University, 9000 Gent, Belgium

${ }^{13}$ II. Physikalisches Institut, Justus-Liebig Universität Gießen, 35392 Gießen, Germany

${ }^{14}$ SUPA, School of Physics and Astronomy, University of Glasgow, Glasgow G12 8QQ, United Kingdom

${ }^{15}$ Department of Physics, University of Illinois, Urbana, Illinois 61801-3080, U.S.A.

${ }^{16}$ Randall Laboratory of Physics, University of Michigan, Ann Arbor, Michigan 48109-1040, U.S.A.

${ }^{17}$ Lebedev Physical Institute, 117924 Moscow, Russia

${ }^{18}$ National Institute for Subatomic Physics (Nikhef), 1009 DB Amsterdam, The Netherlands

${ }^{19}$ Petersburg Nuclear Physics Institute, National Research Center Kurchatov Institute, Gatchina, 188300 Leningrad Region, Russia

${ }^{20}$ Institute for High Energy Physics, National Research Center Kurchatov Institute, Protvino, 142281 Moscow Region, Russia

${ }^{21}$ Institut für Theoretische Physik, Universität Regensburg, 93040 Regensburg, Germany

${ }^{22}$ Istituto Nazionale di Fisica Nucleare, Sezione di Roma, Gruppo Collegato Sanità, and Istituto Superiore di Sanità, 00161 Roma, Italy

${ }^{23}$ TRIUMF, Vancouver, British Columbia V6T 2A3, Canada

${ }^{24}$ Department of Physics, Tokyo Institute of Technology, Tokyo 152, Japan

${ }^{25}$ National Centre for Nuclear Research, 00-689 Warsaw, Poland

${ }^{26}$ Yerevan Physics Institute, 375036 Yerevan, Armenia

E-mail: management@hermes.desy.de

Abstract: A comprehensive set of azimuthal single-spin and double-spin asymmetries in semi-inclusive leptoproduction of pions, charged kaons, protons, and antiprotons from transversely polarized protons is presented. These asymmetries include the previously published HERMES results on Collins and Sivers asymmetries, the analysis of which has been extended to include protons and antiprotons and also to an extraction in a threedimensional kinematic binning and enlarged phase space. They are complemented by corresponding results for the remaining four single-spin and four double-spin asymmetries allowed in the one-photon-exchange approximation of the semi-inclusive deep-inelastic scattering process for target-polarization orientation perpendicular to the direction of the incoming lepton beam. Among those results, significant non-vanishing $\cos \left(\phi-\phi_{S}\right)$ modulations provide evidence for a sizable worm-gear (II) distribution, $g_{1 \mathrm{~T}}^{q}\left(x, \mathbf{p}_{T}^{2}\right)$. Most of the other modulations are found to be consistent with zero with the notable exception of large $\sin \left(\phi_{S}\right)$ modulations for charged pions and $K^{+}$.

KEywords: Fixed target experiments, Polarization, QCD

ArXiv EPrint: 2007.07755 


\section{Contents}

1 Introduction 1

2 TMDs in semi-inclusive deep-inelastic scattering 4

2.1 Structure functions in the semi-inclusive DIS cross section 4

2.2 Connection between structure functions and TMDs 5

2.2.1 The transversity distribution 5

$\begin{array}{lll}2.2 .2 & \text { The Sivers distribution } & 6\end{array}$

2.2.3 The pretzelosity distribution 8

2.2.4 The worm-gear distributions 9

$\begin{array}{ll}2.2 .5 & \text { The subleading Fourier amplitudes } \\ & 10\end{array}$

3 Measurement and analysis $\quad 13$

$\begin{array}{ll}3.1 \text { The HERMES experiment } & 13\end{array}$

$\begin{array}{lll}3.2 & \text { Data selection } & 15\end{array}$

$\begin{array}{ll}\text { 3.3 The extraction of the asymmetry amplitudes } & 19\end{array}$

3.3.1 The choice of the probability-density function 20

$\begin{array}{lll}3.3 .2 & \text { Systematic uncertainties } & 24\end{array}$

$\begin{array}{lll}3.4 & \text { Differences with previous analyses } & 26\end{array}$

4 Results and interpretation $\quad \mathbf{2 6}$

4.1 Signals for transversity and the Collins fragmentation function 28

4.2 Evidence for the Sivers function 33

$\begin{array}{ll}\text { 4.3 The vanishing signals for the pretzelosity function } & 39\end{array}$

4.4 Signals for the worm-gear (II) distribution $g_{1 \mathrm{~T}}^{q}\left(x, \mathbf{p}_{T}^{2}\right) \quad 41$

4.5 The subleading-twist SSA and DSA Fourier amplitudes 42

4.6 Fourier moments arising solely from the longitudinal component of the target polarization $\quad 52$

5 Conclusion $\quad 56$

$\begin{array}{ll}\text { A Contribution from longitudinal target polarization } & 57\end{array}$

B Transverse-momentum factorization and the separation of current and $\begin{array}{ll}\text { target fragmentation } & 61\end{array}$

B.1 Separation of target and current fragmentation 62

$\begin{array}{lll}\text { B.2 Transverse-momentum versus hard scale } & 63\end{array}$

C "Polarizing" Pythia6.2 for the estimate of systematic uncertainties $\quad 66$ 


\section{Introduction}

The present knowledge of the internal structure of the nucleon has emerged from half a century of increasingly precise experimental investigation, in particular of deep-inelastic scattering (DIS) of leptons (see, e.g., refs. [1, 2]). This process is traditionally interpreted in the collinear approximation of the quark-parton model, where the main variable represents the longitudinal momentum of the quark expressed as a fraction $x$ of that of the nucleon, in a frame in which the latter is very large ("infinite-momentum frame"). ${ }^{1}$ One reason for this field to continue flourishing is the intrinsic richness of the subject [3]. Technological advances in polarized beams and targets applied to the deep-inelastic scattering process make it possible to reveal correlations between the spins of both partons and parent nucleon and the longitudinal and transverse components of the momentum of the partons. The key aspects are control of polarizations in the initial state without excessive penalty in luminosity, as well as substantial acceptance permitting detection of not only the scattered leptons but also identified hadrons in the final state. The distribution of these hadrons carries information about the struck quark's transverse momentum, $\mathbf{p}_{T}$, combined with transverse momentum acquired in the fragmentation process, and the type of hadron provides information about the struck quark's flavor.

All parton distribution functions (PDFs) evolve with the hard scale represented in deep-inelastic scattering by $Q^{2}$, where $-Q^{2}$ is the square of the four-momentum of the exchanged virtual photon. ${ }^{2}$ More important in the context of the work presented here is that all PDFs can depend not only on $x$ but also on $\mathbf{p}_{T}$. If the full dependence on these two variables is retained, they are referred to as transverse-momentum dependent (TMD) PDFs.

At leading twist ${ }^{3}$ there are eight TMD PDFs. Only three of them survive integration over $\mathbf{p}_{T}$ and therefore have a corresponding standard collinear PDF: the polarizationaveraged or 'unpolarized' distribution $f_{1}^{q}\left(x, \mathbf{p}_{T}^{2}\right)$, the quark helicity distribution $g_{1}^{q}\left(x, \mathbf{p}_{T}^{2}\right)$, and the transversity distribution $h_{1}^{q}\left(x, \mathbf{p}_{T}^{2}\right)$. While some information is available on the $\mathbf{p}_{T}$ dependence of $f_{1}^{q}\left(x, \mathbf{p}_{T}^{2}\right)$, very little is known about the $\mathbf{p}_{T}$ dependence of the other two.

The five leading-twist TMD PDFs that do not survive integration over $\mathbf{p}_{T}$ typically describe a correlation between $\mathbf{p}_{T}$ and the spin direction of the parent nucleon and/or the ejected quark (and always implicitly $x$ as well). Three of these TMD PDFs are chiral odd $^{4}$ like the transversity distribution, being related to transverse polarization of the struck quark. This property excludes them from influencing any inclusive-DIS observable, at least neglecting mass-suppressed effects. Chiral-odd PDFs appear only in observables involving two chiral-odd partners. Examples of such partnerships are two chiral-odd PDFs in the Drell-Yan process, or a chiral-odd PDF with a chiral-odd fragmentation function (FF) describing production of hadrons in semi-inclusive deep-inelastic scattering.

\footnotetext{
${ }^{1}$ More formally, $x$ is the fraction of the nucleon's light-cone "+" momentum carried by the quark.

${ }^{2}$ For brevity, this dependence will be often omitted in the notation used here.

${ }^{3}$ Following the "working definition" of Jaffe [4], twist $t$ denotes the order $2-t$ of power suppression in the hard scale of the process under study, leading twist corresponding to twist 2 in this context.

${ }^{4}$ The definition of a quark PDF contains two quark fields: chiral-odd functions change sign if the chirality of the field operators is reversed [4].
} 


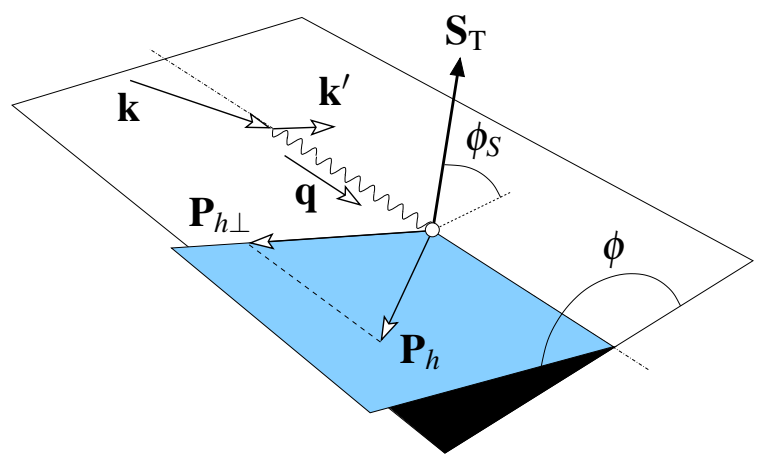

Figure 1. Following the Trento conventions [7], $\phi$ is defined to be the angle between the lepton scattering plane and the plane spanned by the virtual-photon momentum $\mathbf{q} \equiv \mathbf{k}^{\prime}-\mathbf{k}$ (the difference of the momenta of the outgoing and incoming lepton) and $\mathbf{P}_{h}$, the momentum vector of the observed hadron, about the virtual-photon axis. Likewise, the angle $\phi_{S}$ is defined as the angle between the lepton scattering plane and the target-polarization vector $\mathbf{S}_{T}$ of the transversely polarized nucleon.

Two TMD PDFs, the Sivers distribution $f_{1 T}^{\perp, q}\left(x, \mathbf{p}_{T}^{2}\right)$ and the Boer-Mulders distribution $h_{1}^{\perp, q}\left(x, \mathbf{p}_{T}^{2}\right)$ (see section 2.2.2), are rather intriguing because they are odd under naive time reversal (naive- $T$-odd), meaning that they describe a dependence on a triple product of two momenta and a spin vector, which changes sign upon inverting all three-momenta and angular momenta. As will be discussed below, the first observation of a non-zero value for a naive- $T$-odd TMD PDF led to the realization that this property challenges the traditional concepts of factorization and universality of PDFs. Furthermore, the naive- $T$-odd property of TMD PDFs provides a mechanism to explain the otherwise puzzling observation of singlespin asymmetries (SSAs) in either hadron-hadron collisions or deep-inelastic scattering.

There are now indications that a substantial contribution to the helicity sum rule for the nucleon comes from parton orbital angular momentum (cf. refs. [5, 6]). A tantalizing aspect of TMD PDFs is that some of them are related to the orbital angular momentum of quarks. Non-zero values of these TMD PDFs require the presence of nucleon wave function components with different orbital angular momenta. However, no quantitative relationship between a TMD PDF and orbital angular momentum has yet been identified.

TMD PDFs can be experimentally constrained in semi-inclusive deep-inelastic scattering by measurements of azimuthal distributions of the scattered lepton and produced hadrons about the direction of the exchanged virtual photon. The Fourier harmonics of those distributions relate to specific structure functions. The involved angles with respect to the lepton scattering plane are the azimuthal angle $\phi$ of the detected hadron and - when target polarization is involved - the azimuthal angle $\phi_{S}$ of the polarization component orthogonal to the direction of the virtual photon, as depicted in figure 1.

At small transverse momentum, factorization theorems make it possible to express the structure functions as convolutions over quark transverse momentum of a TMD PDF and a TMD FF [3]. TMD PDFs and TMD FFs will collectively be denoted as TMDs, when needed. As final-state polarizations are not measured in the present work, only two leadingtwist TMD FFs are available to couple to the leading-twist TMD PDFs in the structure 


\begin{tabular}{|lccc|}
\hline Name & TMD PDF $/$ FF & Chirality & Naive time reversal \\
\hline Polarization-averaged & $f_{1}^{q}$ & even & even \\
Helicity & $g_{1}^{q}$ & even & even \\
Transversity & $h_{1}^{q}$ & odd & even \\
Sivers & $f_{1 \mathrm{~T}}^{\perp, q}$ & even & odd \\
Boer-Mulders & $h_{1}^{\perp, q}$ & odd & odd \\
Pretzelosity & $h_{1 \mathrm{~T}}^{\perp, q}$ & odd & even \\
Worm-gear (I) & $h_{1 \mathrm{~L}}^{\perp, q}$ & odd & even \\
Worm-gear (II) & $g_{1 \mathrm{~T}}^{q}$ & even & even \\
\hline Polarization-averaged & $D_{1}^{q \rightarrow h}$ & even & even \\
Collins & $H_{1}^{\perp, q \rightarrow h}$ & odd & odd \\
\hline
\end{tabular}

Table 1. Leading-twist TMD distribution and fragmentation functions and their key symmetry properties. Only the first three TMD PDFs and $D_{1}^{q \rightarrow h}$ survive integration over transverse momentum.

functions. The chiral-even TMD PDFs are convoluted with the polarization-averaged TMD FF $D_{1}^{q \rightarrow h}\left(z, z^{2} \mathbf{k}_{T}^{2}\right)$, while the chiral-odd TMD PDFs, such as the transversity distribution, are convoluted with the chiral-odd naive- $T$-odd Collins TMD FF $H_{1}^{\perp, q \rightarrow h}\left(z, z^{2} \mathbf{k}_{T}^{2}\right)$, representing a correlation between the transverse polarization of the fragmenting quark and the transverse momentum $z \mathbf{k}_{T}$ of the produced hadron carrying the fraction $z$ of the energy of the virtual photon in the target-rest frame. Thus, the Collins fragmentation function acts as a 'quark polarimeter'. Table 1 summarizes some properties of the leading-twist TMDs.

In this work, azimuthal asymmetries in the yield of pions and charged kaons are extracted from semi-inclusive deep-inelastic scattering data recorded with a transversely polarized hydrogen target at the HERMES experiment. They are supplemented with the first such measurements for proton and antiproton electroproduction. Fourier amplitudes of single-spin asymmetries are presented that arise from the transversity $h_{1}^{q}\left(x, \mathbf{p}_{T}^{2}\right)$, the Sivers $f_{1 \mathrm{~T}}^{\perp, q}\left(x, \mathbf{p}_{T}^{2}\right)$, and the pretzelosity $h_{1 \mathrm{~T}}^{\perp, q}\left(x, \mathbf{p}_{T}^{2}\right)$ distributions. Also, a Fourier amplitude related to the worm-gear distribution $g_{1 \mathrm{~T}}^{q}\left(x, \mathbf{p}_{T}^{2}\right)$ is extracted from the double-spin asymmetry (DSA) requiring longitudinally polarized beams. Besides these leading-twist contributions, kinematically suppressed Fourier amplitudes are also reported, e.g., those related to the other worm-gear distribution $h_{1 \mathrm{~L}}^{\perp, q}\left(x, \mathbf{p}_{T}^{2}\right)$ due to a small longitudinal component of the target-polarization vector (cf. appendix A), or those involving twist-3 TMDs. All Fourier amplitudes for charged mesons and for protons are extracted in a three-dimensional binning in the kinematic variables $x, z$, as well as the transverse hadron momentum, which will greatly facilitate disentangling the underlying dynamics of the partonic nucleon structure and of the fragmentation process. The Fourier amplitudes are extracted also in onedimensional binning in those variables. Due to insufficient yields, the Fourier amplitudes for neutral pions and for antiprotons are provided in only the one-dimensional binning. 


\section{TMDs in semi-inclusive deep-inelastic scattering}

\subsection{Structure functions in the semi-inclusive DIS cross section}

The observables of interest in this work are Fourier amplitudes of the semi-inclusive DIS cross section, selected in all cases by the polarization direction of the target nucleon with respect to the direction of the virtual photon, and - in some cases - also by the helicity of the beam lepton. The azimuthal dependence of the cross section for leptoproduction of hadrons on a nucleon $N$ can be decomposed in the one-photon-exchange approximation in terms of semi-inclusive DIS structure functions as [8]

$$
\begin{aligned}
& \frac{\mathrm{d} \sigma^{l N \rightarrow l h X}}{\mathrm{~d} x \mathrm{~d} y \mathrm{~d} \phi_{S} \mathrm{~d} z \mathrm{~d} \phi \mathrm{d} P_{h \perp}^{2}} \propto\left\{F_{\mathrm{UU}, \mathrm{T}}+\epsilon F_{\mathrm{UU}, \mathrm{L}}\right. \\
& +\sqrt{2 \epsilon(1+\epsilon)} \cos (\phi) F_{\mathrm{UU}}^{\cos (\phi)}+\epsilon \cos (2 \phi) F_{\mathrm{UU}}^{\cos (2 \phi)}+\lambda_{l} \sqrt{2 \epsilon(1-\epsilon)} \sin (\phi) F_{\mathrm{LU}}^{\sin (\phi)} \\
& +S_{L}\left[\sqrt{2 \epsilon(1+\epsilon)} \sin (\phi) F_{\mathrm{UL}}^{\sin (\phi)}+\epsilon \sin (2 \phi) F_{\mathrm{UL}}^{\sin (2 \phi)}\right] \\
& +S_{L} \lambda_{l}\left[\sqrt{1-\epsilon^{2}} F_{\mathrm{LL}}+\sqrt{2 \epsilon(1-\epsilon)} \cos (\phi) F_{\mathrm{LL}}^{\cos (\phi)}\right] \\
& +S_{T}\left[\sin \left(\phi-\phi_{S}\right)\left(F_{\mathrm{UT}, \mathrm{T}}^{\sin \left(\phi-\phi_{S}\right)}+\epsilon F_{\mathrm{UT}, \mathrm{L}}^{\sin \left(\phi-\phi_{S}\right)}\right)\right. \\
& +\epsilon \sin \left(\phi+\phi_{S}\right) F_{\mathrm{UT}}^{\sin \left(\phi+\phi_{S}\right)}+\epsilon \sin \left(3 \phi-\phi_{S}\right) F_{\mathrm{UT}}^{\sin \left(3 \phi-\phi_{S}\right)} \\
& \left.+\sqrt{2 \epsilon(1+\epsilon)} \sin \left(\phi_{S}\right) F_{\mathrm{UT}}^{\sin \left(\phi_{S}\right)}+\sqrt{2 \epsilon(1+\epsilon)} \sin \left(2 \phi-\phi_{S}\right) F_{\mathrm{UT}}^{\sin \left(2 \phi-\phi_{S}\right)}\right] \\
& +S_{T} \lambda_{l}\left[\sqrt{1-\epsilon^{2}} \cos \left(\phi-\phi_{S}\right) F_{\mathrm{LT}}^{\cos \left(\phi-\phi_{S}\right)}\right. \\
& \left.\left.+\sqrt{2 \epsilon(1-\epsilon)} \cos \left(\phi_{S}\right) F_{\mathrm{LT}}^{\cos \left(\phi_{S}\right)}+\sqrt{2 \epsilon(1-\epsilon)} \cos \left(2 \phi-\phi_{S}\right) F_{\mathrm{LT}}^{\cos \left(2 \phi-\phi_{S}\right)}\right]\right\},
\end{aligned}
$$

where $x \equiv Q^{2} /(2 P \cdot q),{ }^{5} y \equiv(P \cdot q) /(P \cdot k), z \equiv\left(P \cdot P_{h}\right) /(P \cdot q)$, with $q, P, k, k^{\prime}$ and $P_{h}$ representing the four-momenta of the exchanged virtual photon, initial-state target nucleon, incident and outgoing lepton, and produced hadron $h$, respectively. Furthermore,

$$
P_{h \perp} \equiv\left|\mathbf{P}_{h}-\frac{\left(\mathbf{P}_{h} \cdot \mathbf{q}\right) \mathbf{q}}{|\mathbf{q}|^{2}}\right|
$$

is the magnitude of the hadron's transverse momentum, $\lambda_{l}=+1\left(\lambda_{l}=-1\right)$ denotes righthanded (left-handed) beam leptons in the lepton-nucleon center-of-mass system, and the

\footnotetext{
${ }^{5}$ While the right-hand side of this equation corresponds to the Bjorken variable, it coincides with the light-cone momentum fraction introduced in section 1 in the Bjorken limit.
} 
"photon polarization parameter" $\epsilon \equiv \frac{1-y-\frac{1}{4} \gamma^{2} y^{2}}{1-y+\frac{1}{4} y^{2}\left(\gamma^{2}+2\right)}$ is the ratio of longitudinal to transverse photon flux, where $\gamma \equiv 2 M x / Q$ with $M$ the mass of the target nucleon.

The structure functions $F$ depend in general on $x, z, P_{h \perp}$ and $Q^{2}$. The first subscript $\mathrm{U}(\mathrm{L})$ on the structure functions represents unpolarized (longitudinally polarized) beam, while the second subscript T (L) denotes transverse (longitudinal) target polarization $S_{T}$ $\left(S_{L}\right)$. When present, the third subscript $\mathrm{T}(\mathrm{L})$ denotes transverse (longitudinal) virtual photons. In principle, all structure functions have a dependence on the hadron type, although the hadron label $h$ is omitted for compactness.

As will be discussed in more detail in section 2.2, the transverse-polarization-dependent azimuthal modulations appearing in the fifth, sixth, and eighth line of eq. (2.1) arise as convolutions of leading-twist (twist-2) TMDs, while the remaining transverse-polarization dependent terms involve twist-3 TMDs.

\subsection{Connection between structure functions and TMDs}

According to factorization theorems (see, e.g., refs. [3, 9-15] and references therein), at small transverse momentum ${ }^{6}$ the structure functions in eq. (2.1) can be written as convolutions in transverse-momentum space of a TMD PDF and a TMD FF, possibly accompanied by a weighting factor $w\left(\mathbf{p}_{T}, \mathbf{k}_{T}\right)$, i.e.,

$$
F\left(x, z, P_{h \perp}, Q^{2}\right)=\mathcal{C}\left[w f^{q} D^{q \rightarrow h}\right],
$$

where the notation $\mathcal{C}$ indicates the convolution

$$
\begin{aligned}
\mathcal{C}\left[w f^{q} D^{q \rightarrow h}\right] \equiv \sum_{q} e_{q}^{2} H\left(Q^{2}\right) \int \mathrm{d}^{2} \mathbf{p}_{T} \mathrm{~d}^{2} \mathbf{k}_{T} \delta^{(2)}\left(\mathbf{p}_{T}-\mathbf{k}_{T}-\frac{\mathbf{P}_{h \perp}}{z}\right) \\
w\left(\mathbf{p}_{T}, \mathbf{k}_{T}\right) f^{q}\left(x, \mathbf{p}_{T}^{2} ; Q^{2}\right) D^{q \rightarrow h}\left(z, \mathbf{k}_{T}^{2} ; Q^{2}\right) .
\end{aligned}
$$

Here, $e_{q}$ are the quark electric charges in units of the elementary charge, $H$ is a hard function that can be computed perturbatively as a power expansion in the strong coupling constant $\alpha_{S}$ [15]. The TMDs included in the convolution depend on $Q^{2}$ in a way dictated by TMD evolution equations $[17-19] .{ }^{7}$ At variance with collinear PDFs, TMD evolution contains a universal, flavor- and spin-independent nonperturbative component, which has to be fixed from data [20-22] or computed in lattice QCD [23-25]. At partonmodel level, the hard function reduces to unity, the TMDs become independent of $Q^{2}$ and the convolutions correspond to the definition in, e.g., ref. [8].

Table 2 summarizes the correspondence between the leading-twist azimuthal modulations defined in eq. (2.1) and the TMDs appearing in the structure-function expressions. Further details are provided below.

\subsubsection{The transversity distribution}

The transversity distribution has the probabilistic interpretation as the difference in number densities of quarks with transverse polarization parallel and anti-parallel to the transverse

\footnotetext{
${ }^{6}$ See ref. [16] and appendix B for a discussion on the limits of applicability of the TMD formalism.

${ }^{7}$ TMDs depend on two scales, usually denoted as $\mu^{2}$ and $\zeta$, but for simplicity we set them both to be equal to the hard scale $Q^{2}$.
} 


\begin{tabular}{|lccc|}
\hline Name & TMD PDF & TMD FF & Structure function \\
\hline Transversity & $h_{1}^{q}$ & $H_{1}^{\perp, q \rightarrow h}$ & $F_{\mathrm{UT}}^{\sin \left(\phi+\phi_{S}\right)}$ \\
Sivers & $f_{1, q}^{\perp, q}$ & $D_{1}^{q \rightarrow h}$ & $F_{\mathrm{UT}}^{\sin \left(\phi-\phi_{S}\right)}$ \\
Boer-Mulders & $h_{1}^{\perp, q}$ & $H_{1}^{\perp, q \rightarrow h}$ & $F_{\mathrm{UU}}^{\cos (2 \phi)}$ \\
Pretzelosity & $h_{1 \mathrm{~T}}^{\perp, q}$ & $H_{1}^{\perp, q \rightarrow h}$ & $F_{\mathrm{UT}}^{\sin \left(3 \phi-\phi_{S}\right)}$ \\
Worm-gear (I) & $h_{1 \mathrm{~L}}^{\perp, q}$ & $H_{1}^{\perp, q \rightarrow h}$ & $F_{\mathrm{UL}}^{\sin (2 \phi)}$ \\
Worm-gear (II) & $g_{1 \mathrm{~T}}^{q}$ & $D_{1}^{q \rightarrow h}$ & $F_{\mathrm{LT}}^{\cos \left(\phi-\phi_{S}\right)}$ \\
\hline
\end{tabular}

Table 2. Leading-twist TMD PDFs that do not survive integration over $\mathbf{p}_{T}$, together with the TMD FFs with which they appear in their associated leading semi-inclusive DIS structure functions.

polarization of the parent nucleon [26]. Among the three leading-twist PDFs surviving integration over $\mathbf{p}_{T}$, it is the only one that involves transverse quark polarization and is thereby chiral-odd. Unlike the polarization-averaged and the quark-helicity distributions, QCD evolution of the transversity in a spin- $\frac{1}{2}$ hadron does not mix quarks with gluons because of helicity conservation [27].

The transversity distribution $h_{1}^{q}$ appears together with the Collins fragmentation function $H_{1}^{\perp, q \rightarrow h}$ in the structure function

$$
F_{\mathrm{UT}}^{\sin \left(\phi+\phi_{S}\right)}\left(x, z, P_{h \perp}, Q^{2}\right)=\mathcal{C}\left[-\frac{\hat{\mathbf{h}} \cdot \mathbf{k}_{T}}{M_{h}} h_{1}^{q} H_{1}^{\perp, q \rightarrow h}\right],
$$

where $M_{h}$ is the mass of the produced hadron and $\hat{\mathbf{h}}=\mathbf{P}_{h \perp} /\left|\mathbf{P}_{h \perp}\right|$.

Azimuthal asymmetries related to $F_{\mathrm{UT}}^{\sin \left(\phi+\phi_{S}\right)}$ as a function of single kinematic variables have been published by the HERMES Collaboration for charged pions [28] and later for pions, charged kaons, as well as the pion charge-difference [29], all from a transversely polarized hydrogen target. In the present work, the three-dimensional dependences of the so-called Collins asymmetries go beyond the original works of refs. [28, 29], which concentrated on one-dimensional binning in either the kinematic variable $x, z$, or $P_{h \perp}$. In addition, results for protons and antiprotons obtained here for the first time are included.

\subsubsection{The Sivers distribution}

The Sivers and Boer-Mulders functions are the only TMDs that are naive- $T$-odd. The chiral-even Sivers function $f_{1 \mathrm{~T}}^{\perp, q}$ [30] has the probabilistic interpretation as the dependence of the number density of quarks on the orientation of $\mathbf{p}_{T}$ with respect to the transverse polarization of the parent nucleon, while the chiral-odd Boer-Mulders function $h_{1}^{\perp, q}$ [31] relates $\mathbf{p}_{T}$ to the transverse polarization of the struck quark in an unpolarized nucleon. The Boer-Mulders function is not further discussed in this paper, but relevant measurements and discussions are reported in refs. [32, 33] and the references therein.

Among the TMDs that do not survive integration over $\mathbf{p}_{T}$, these naive- $T$-odd functions have thus far received the most attention, both experimentally and theoretically. The possible existence of the Sivers function was proposed already three decades ago [30] in an effort 
to explain the unexpected single-spin asymmetries that had appeared in the production of pions from the collision of unpolarized with transversely polarized protons [34]. That interpretation came under doubt when the naive- $T$-odd Collins fragmentation function was proposed as an alternative mechanism, and it was demonstrated that the existence of such naive- $T$-odd TMD PDFs would violate the fundamental time reversal symmetry [35].

A flurry of theoretical activity was inspired by a seminal model calculation [36] showing how the Sivers function could legitimately arise through overlap integrals of quark wave functions with different orbital angular momenta, together with a final-state interaction of the ejected quark with the target remnant. This soon led to the realization $[35,37$, $38]$ that the aforementioned demonstration applied only to $\mathbf{p}_{T}$-integrated PDFs, in the definition of which a gauge link in the final state could legitimately be neglected. The gauge-invariant definition of TMD PDFs requires this gauge link, which then provides the phase necessary for the interference associated with the naive- $T$-odd property. The link can be interpreted as a final-state interaction of the ejected quark with the color field of the target remnant. This interaction can be considered to be embodied in the TMD PDF itself, with $\mathbf{p}_{T}$ representing the transverse momentum following the interaction [39].

Incorporation of the gauge link into factorization proofs had a profound impact. The concept of universality of leading-twist distribution functions had to be generalized to allow for specific interaction dependences. In the case of the Sivers function, and in fact for all naive- $T$-odd TMDs, they are predicted to appear with the opposite sign in the expressions for deep-inelastic scattering and Drell-Yan cross sections [35], reflecting the appearance of the embodied interaction in the final or initial state, respectively. ${ }^{8}$ While the existence of a nonzero Sivers function was finally firmly established by data for semiinclusive deep-inelastic scattering of leptons with transversely polarized targets [28, 40, 41], the experimental verification of this direct prediction of QCD is eagerly awaited. Recent measurements of transverse-spin asymmetries in weak-boson production and the Drell-Yan process [42, 43], albeit not sufficiently precise, are consistent with the sign change predicted.

Much of the interest in the Sivers function arises from the evidence linking it to orbital angular momentum of quarks. Model calculations have found quark wave function components with differing orbital angular momenta to be necessary for a non-zero Sivers function. The same statement can be made for relativistic theories of the anomalous magnetic moment $\kappa$ of the nucleon. In fact, the same wave function components appear in both cases [44]. Under certain plausible assumptions, such as an attractive final-state interaction, the sign of the Sivers function for each quark flavor is related to the sign of the contribution of this flavor to $\kappa$ [45]. The predicted relationship is consistent with experiment [40]. A quantitative estimate of orbital angular momentum based on the Sivers function was attempted [46], but it was based on restrictive assumptions [47].

In semi-inclusive deep-inelastic scattering, the Sivers function appears convoluted with the unpolarized fragmentation function in the structure function [31]

$$
F_{\mathrm{UT}}^{\sin \left(\phi-\phi_{S}\right)}\left(x, z, P_{h \perp}, Q^{2}\right)=\mathcal{C}\left[-\frac{\hat{\mathbf{h}} \cdot \mathbf{p}_{T}}{M} f_{1 \mathrm{~T}}^{\perp, q} D_{1}^{q \rightarrow h}\right] .
$$

\footnotetext{
${ }^{8}$ In the context of the present work, these distributions should therefore in principle appear with the label 'DIS'.
} 
It should be noted that the $\sin \left(\phi-\phi_{S}\right)$ modulation of the semi-inclusive DIS cross section is the only one, besides the azimuthally uniform denominator of all the SSA and DSA amplitudes, that can in principle receive contributions from longitudinally polarized photons; these contributions, however, are vanishing at leading and subleading twist in the region of low transverse momentum.

The HERMES Collaboration presented results for closely related asymmetries for identified pions and charged kaons, as well as for the pion charge-difference asymmetry from a transversely polarized hydrogen target $[28,40]$. In the present work, the threedimensional dependences go again beyond those original works, which concentrated on one-dimensional kinematic binning in either $x, z$, or $P_{h \perp}$. Furthermore, results for protons and antiprotons are presented here for the first time.

\subsubsection{The pretzelosity distribution}

The naive- $T$-even chiral-odd pretzelosity TMD $h_{1 \mathrm{~T}}^{\perp, q}$, introduced for the first time by Mulders and Tangerman [48], has the probabilistic interpretation as the dependence of the number density of quarks on the relative orientation of $\mathbf{p}_{T}$ and the transverse polarizations of both the quark and parent nucleon. In a helicity basis, this tensor structure corresponds to a flip of the quark helicity and nucleon helicity in opposite directions. The struck quark therefore has to absorb two units of orbital angular momentum $L_{z}$, requiring either the presence of $s-d$ interference in the nucleon wave function, or matrix elements that are quadratic in a $p$ wave component. Unlike all other leading-twist TMDs, pretzelosity receives no perturbative contributions at large transverse momentum up to order $\alpha_{S}^{2}$ [49, 50]. Other properties of the pretzelosity distribution are given, e.g., in ref. [51]. In various models, such as bag or spectator models, the pretzelosity distribution appears as the difference between helicity and transversity distributions, and hence can be interpreted as representing relativistic effects in the nucleon structure. ${ }^{9}$ The name pretzelosity is loosely connected to the fact that this TMD is related to a quadrupolar distortion of the quark density $[52,53]$.

Being chiral-odd, pretzelosity appears in semi-inclusive DIS convoluted with the Collins fragmentation function leading to a $\sin \left(3 \phi-\phi_{S}\right)$ modulation of the cross section $[48,54]$ :

$$
\begin{aligned}
F_{\mathrm{UT}}^{\sin \left(3 \phi-\phi_{S}\right)} & \left(x, z, P_{h \perp}, Q^{2}\right)= \\
\mathcal{C} & {\left[\frac{2\left(\hat{\mathbf{h}} \cdot \mathbf{p}_{T}\right)\left(\mathbf{p}_{T} \cdot \mathbf{k}_{T}\right)+\mathbf{p}_{T}^{2}\left(\hat{\mathbf{h}} \cdot \mathbf{k}_{T}\right)-4\left(\hat{\mathbf{h}} \cdot \mathbf{p}_{T}\right)^{2}\left(\hat{\mathbf{h}} \cdot \mathbf{k}_{T}\right)}{2 M^{2} M_{h}} h_{1 \mathrm{~T}}^{\perp, q} H_{1}^{\perp, q \rightarrow h}\right] . }
\end{aligned}
$$

The only existing measurement of this asymmetry for identified hadrons comes from the Jefferson Lab Hall A Collaboration [55]; a transversely polarized ${ }^{3} \mathrm{He}$ target was used, effectively a target of transversely polarized neutrons. The resulting asymmetry amplitudes are consistent with zero, both for $\pi^{+}$and $\pi^{-}$. The COMPASS Collaboration presented vanishing asymmetry amplitudes for unidentified hadrons using a transversely polarized $\mathrm{NH}_{3}$ target [56]. The measurements presented here for pions, charged kaons as well as for

\footnotetext{
${ }^{9}$ For a non-relativistic system, where boosts and rotations commute, the transversity and helicity distributions would coincide (cf. ref. [4]).
} 
protons and antiprotons are the first of their kind for scattering off transversely polarized protons.

\subsubsection{The worm-gear distributions}

The TMD PDFs $g_{1 \mathrm{~T}}^{q}\left(x, \mathbf{p}_{T}^{2}\right)$ and $h_{1 \mathrm{~L}}^{\perp, q}\left(x, \mathbf{p}_{T}^{2}\right)[48,57,58]$ respectively describe the number density of longitudinally polarized quarks in a transversely polarized nucleon and of transversely polarized quarks in a longitudinally polarized nucleon. The name "worm gear" refers to the orthogonal orientation of the spins of quarks and nucleons. Both distributions are naive- $T$-even, and $g_{1 \mathrm{~T}}^{q}\left(x, \mathbf{p}_{T}^{2}\right)$ is chiral-even while $h_{1 \mathrm{~L}}^{\perp, q}\left(x, \mathbf{p}_{T}^{2}\right)$ is chiral-odd.

A feature that distinguishes the two worm-gear distributions from all other TMDs is that, in light-cone quark models, the corresponding generalized parton distributions (GPDs) vanish [59]. Furthermore, model calculations [60] find that the two distributions are closely related: $g_{1 \mathrm{~T}}^{q}\left(x, \mathbf{p}_{T}^{2}\right)=-h_{1 \mathrm{~L}}^{\perp, q}\left(x, \mathbf{p}_{T}^{2}\right)$. However, this cannot be generally true at all scales due to the different evolution of chiral-even versus chiral-odd distributions (cf. ref. [61]).

In the Wandzura-Wilczek-type approximation (see, e.g., [62]), relations can be established between the worm-gear distributions $g_{1 \mathrm{~T}}^{q}\left(x, \mathbf{p}_{T}^{2}\right)$ and $h_{1 \mathrm{~L}}^{\perp, q}\left(x, \mathbf{p}_{T}^{2}\right)$ and the helicity and transversity distributions, respectively ${ }^{10}$

$$
\begin{gathered}
g_{1 \mathrm{~T}}^{(1), q} \equiv \int \mathrm{d} \mathbf{p}_{T}^{2} \frac{\mathbf{p}_{T}^{2}}{2 M^{2}} g_{1 \mathrm{~T}}^{q}\left(x, \mathbf{p}_{T}^{2}\right) \stackrel{\mathrm{WW}}{\approx} x \int_{x}^{1} \frac{\mathrm{d} \xi}{\xi} g_{1}^{q}(\xi) \stackrel{\mathrm{WW}}{\approx} x g_{\mathrm{T}}^{q}, \\
h_{1 \mathrm{~L}}^{\perp(1), q} \equiv \int \mathrm{d} \mathbf{p}_{T}^{2} \frac{\mathbf{p}_{T}^{2}}{2 M^{2}} h_{1 \mathrm{~L}}^{\perp, q}\left(x, \mathbf{p}_{T}^{2}\right) \stackrel{\mathrm{WW}}{\approx}-x^{2} \int_{x}^{1} \frac{\mathrm{d} \xi}{\xi^{2}} h_{1}^{q}(\xi) \stackrel{\mathrm{WW}}{\approx}-\frac{1}{2} x h_{\mathrm{L}}^{q},
\end{gathered}
$$

where all approximate signs involve Wandzura-Wilczek-type approximations and the neglect of mass terms. Experimental tests of the relations between the $\mathbf{p}_{T}^{2}$-moments of the worm-gear and the particular moments of the collinear helicity and transversity distributions would thus provide indications whether or not the relevant genuine twist- 3 contributions to $g_{\mathrm{T}}$ and $h_{\mathrm{L}}$ are significant (cf. discussion in ref. [65]).

The structure function $F_{\mathrm{LT}}^{\cos \left(\phi-\phi_{S}\right)}$ of the target-spin and beam-helicity dependent cross section provides a leading-twist signal for the worm-gear (II) distribution $g_{1 \mathrm{~T}}^{q}\left(x, \mathbf{p}_{T}^{2}\right)$ in conjunction with the polarization-averaged fragmentation function $D_{1}^{q \rightarrow h}\left(z, z^{2} \mathbf{k}_{T}^{2}\right)$ :

$$
F_{\mathrm{LT}}^{\cos \left(\phi-\phi_{S}\right)}\left(x, z, P_{h \perp}, Q^{2}\right)=\mathcal{C}\left[\frac{\hat{\mathbf{h}} \cdot \mathbf{p}_{T}}{M} g_{1 \mathrm{~T}}^{q} D_{1}^{q \rightarrow h}\right]
$$

The Jefferson Lab Hall A Collaboration published related results for charged pions produced in semi-inclusive deep-inelastic scattering off transversely polarized ${ }^{3} \mathrm{He}$ and used these data to extract the corresponding Fourier amplitude for transversely polarized neutrons [66]. While the results for positive pions are consistent with zero, the ones for negative pions provide first evidence for a non-vanishing $g_{1 \mathrm{~T}}^{q}\left(x, \mathbf{p}_{T}^{2}\right)$. The measurements presented

\footnotetext{
${ }^{10}$ For the adaptation of the original Wandzura-Wilczek approximation [63] to semi-inclusive DIS see [64] and references therein.
} 
here for pions, charged kaons as well as for protons and antiprotons are the first of their kind for transversely polarized protons.

The chiral-odd worm-gear (I) distribution $h_{1 \mathrm{~L}}^{\perp, q}\left(x, \mathbf{p}_{T}^{2}\right)$ couples to the chiral-odd Collins fragmentation function. In semi-inclusive deep-inelastic scattering from longitudinally polarized nucleons this combination gives rise to $[48,54]$

$$
F_{\mathrm{UL}}^{\sin (2 \phi)}\left(x, z, P_{h \perp}, Q^{2}\right)=\mathcal{C}\left[-\frac{2\left(\hat{\mathbf{h}} \cdot \mathbf{k}_{T}\right)\left(\hat{\mathbf{h}} \cdot \mathbf{p}_{T}\right)-\mathbf{k}_{T} \cdot \mathbf{p}_{T}}{M M_{h}} h_{1 \mathrm{~L}}^{\perp, q} H_{1}^{\perp, q \rightarrow h}\right] .
$$

The primary choice for studying $F_{\mathrm{UL}}^{\sin (2 \phi)}$ is scattering off a longitudinally polarized target (cf. ref. [67]), as such it would not normally be included in the present measurement. However, due to the small but non-vanishing target-spin component that is longitudinal to the virtual-photon direction in measurements on targets polarized perpendicular to the incident-beam direction (cf. appendix A), the worm-gear (II) distribution $h_{1 \mathrm{~L}}^{\perp, q}\left(x, \mathbf{p}_{T}^{2}\right)$ can in principle be constrained also by these data. This will be further discussed in the corresponding section 4.6 .

\subsubsection{The subleading Fourier amplitudes}

Each structure function in both the antepenultimate and the ultimate lines of eq. (2.1) is given by a sum of several terms, each of which contains a twist-3 TMD convoluted with a twist-2 TMD. The twist-3 objects have no probabilistic interpretation and contain interaction-dependent terms, i.e., they involve quark-gluon correlations in the nucleon wave function. All these terms are suppressed by the factor $(M / Q)$, and hence become negligible in the Bjorken limit. Nevertheless, evidences for substantial twist-3 contributions to singlespin asymmetries have already been found in the HERMES kinematic region [67-72]. The $\sin \phi$ Fourier amplitude of the $\pi^{+}$leptoproduction cross section for longitudinally polarized protons was found to have magnitudes as large as about $5 \%$ of the polarization-averaged cross section, which are typical of the more sizable leading-twist Fourier amplitudes among those mentioned above. Hence, it is of interest to also extract here the non-leading singlespin and double-spin asymmetries for transverse target polarization.

The $F_{\mathrm{UT}}^{\sin \left(2 \phi-\phi_{S}\right)}$ structure function is given by

$$
\begin{aligned}
F_{\mathrm{UT}}^{\sin \left(2 \phi-\phi_{S}\right)} & \left(x, z, P_{h \perp}, Q^{2}\right)= \\
& \frac{2 M}{Q} \mathcal{C}\left[\frac{2\left(\hat{\mathbf{h}} \cdot \mathbf{p}_{T}\right)^{2}-\mathbf{p}_{T}^{2}}{2 M^{2}}\left(x f_{\mathrm{T}}^{\perp, q} D_{1}^{q \rightarrow h}-\frac{M_{h}}{z M} h_{1 \mathrm{~T}}^{\perp, q} \widetilde{H}^{q \rightarrow h}\right)\right. \\
& -\frac{2\left(\hat{\mathbf{h}} \cdot \mathbf{k}_{T}\right)\left(\hat{\mathbf{h}} \cdot \mathbf{p}_{T}\right)-\mathbf{p}_{T} \cdot \mathbf{k}_{T}}{2 M M_{h}} \times \\
& \left.\left(x h_{\mathrm{T}}^{q} H_{1}^{\perp, q \rightarrow h}+\frac{M_{h}}{z M} g_{1 \mathrm{~T}}^{q} \widetilde{G}^{\perp, q \rightarrow h}+x h_{\mathrm{T}}^{\perp, q} H_{1}^{\perp, q \rightarrow h}-\frac{M_{h}}{z M} f_{1 \mathrm{~T}}^{\perp, q} \widetilde{D}^{\perp, q \rightarrow h}\right)\right] .
\end{aligned}
$$


The interaction-dependent fragmentation functions are indicated by a tilde. Similarly, the $F_{\mathrm{UT}}^{\sin \left(\phi_{S}\right)}$ structure function is given by

$$
\begin{gathered}
F_{\mathrm{UT}}^{\sin \left(\phi_{S}\right)}\left(x, z, P_{h \perp}, Q^{2}\right)=\frac{2 M}{Q} \mathcal{C}\left[x f_{\mathrm{T}}^{q} D_{1}^{q \rightarrow h}-\frac{M_{h}}{z M} h_{1}^{q} \widetilde{H}^{q \rightarrow h}-\frac{\mathbf{p}_{T} \cdot \mathbf{k}_{T}}{2 M M_{h}} \times\right. \\
\left.\left(x h_{\mathrm{T}}^{q} H_{1}^{\perp, q \rightarrow h}+\frac{M_{h}}{z M} g_{1 \mathrm{~T}}^{q} \widetilde{G}^{\perp, q \rightarrow h}-x h_{\mathrm{T}}^{\perp, q} H_{1}^{\perp, q \rightarrow h}+\frac{M_{h}}{z M} f_{1 \mathrm{~T}}^{\perp, q} \widetilde{D}^{\perp, q \rightarrow h}\right)\right] .
\end{gathered}
$$

The two structure functions involve rather similar combinations of twist-2 and twist-3 distribution and fragmentation functions. In Wandzura-Wilczek-type approximations, the chiral-even naive- $T$-odd twist- 3 distributions $f_{\mathrm{T}}^{q}$ and $f_{\mathrm{T}}^{\perp, q}$ are related to the Sivers function, while the difference (sum) of the chiral-odd naive- $T$-even twist-3 distributions $h_{\mathrm{T}}^{q}$ and $h_{\mathrm{T}}^{\perp, q}$ are related to the transversity (pretzelosity) [8]. In general, the interaction-dependent fragmentation functions disappear in the Wandzura-Wilczek-type approximation. The expressions for these two structure functions thus simplify significantly in such an approach [64].

A unique feature of the partial cross section given by eq. (2.13) is that it is the only contribution to the cross section $\sigma_{\mathrm{UT}}^{h}$ that survives integration over transverse hadron momentum $[8,73]$ :

$$
\int d^{2} \mathbf{P}_{h \perp} F_{\mathrm{UT}}^{\sin \left(\phi_{S}\right)}\left(x, z, P_{h \perp}, Q^{2}\right)=-x \frac{2 M_{h}}{Q} \sum_{q} e_{q}^{2} h_{1}^{q}(x) \frac{\widetilde{H}^{q \rightarrow h}(z)}{z} .
$$

It thus provides sensitivity to the transversity distribution without involving a convolution over intrinsic transverse momenta. Nonetheless, due to time-reversal invariance, this modulation must vanish in the one-photon-exchange approximation in the inclusive limit [74], i.e., summing over all final-state hadrons and integrating over $z$, which indeed was demonstrated in the kinematic regime of this measurement in ref. [75].

Interest in $\tilde{H}^{q \rightarrow h}(z)$ has grown significantly in the past years due to its connection to the single-spin asymmetries observed in $p^{\uparrow} p \rightarrow \pi X$. Using Lorentz-invariance relations as well as QCD equations of motion, it has been shown that both $\tilde{H}^{q \rightarrow h}(z)$ and the Collins function arise from the same underlying dynamical correlator [76, 77]. As a consequence, it would be very surprising if this function vanished. Besides being a candidate for explaining single-spin asymmetries observed in $p^{\uparrow} p \rightarrow \pi X$ (cf. ref. [78] and references therein), it also contributes to transverse target single-spin asymmetries in inclusive electroproduction of hadrons [79] as measured, e.g., at HERMES [80] or Jefferson Lab [81].

Finally, the subleading structure functions contributing to the cross section $\sigma_{\mathrm{LT}}^{h}$ are given by

$$
\begin{aligned}
& F_{\mathrm{LT}}^{\cos \left(2 \phi-\phi_{S}\right)}\left(x, z, P_{h \perp}, Q^{2}\right)= \\
& \frac{2 M}{Q} \mathcal{C}\left[-\frac{2\left(\hat{\mathbf{h}} \cdot \mathbf{p}_{T}\right)^{2}-\mathbf{p}_{T}^{2}}{2 M^{2}}\left(x g_{\mathrm{T}}^{\perp, q} D_{1}^{q \rightarrow h}+\frac{M_{h}}{z M} h_{1 \mathrm{~T}}^{\perp, q} \widetilde{E}^{q \rightarrow h}\right)+\frac{2\left(\hat{\mathbf{h}} \cdot \mathbf{k}_{T}\right)\left(\hat{\mathbf{h}} \cdot \mathbf{p}_{T}\right)-\mathbf{p}_{T} \cdot \mathbf{k}_{T}}{2 M M_{h}} \times\right. \\
& \left.\quad\left(x e_{\mathrm{T}}^{q} H_{1}^{\perp, q \rightarrow h}-\frac{M_{h}}{z M} g_{1 \mathrm{~T}}^{q} \widetilde{D}^{\perp, q \rightarrow h}-x e_{\mathrm{T}}^{\perp, q} H_{1}^{\perp, q \rightarrow h}-\frac{M_{h}}{z M} f_{1 \mathrm{~T}}^{\perp, q} \widetilde{G}^{\perp, q \rightarrow h}\right)\right],
\end{aligned}
$$


and

$$
\begin{gathered}
F_{\mathrm{LT}}^{\cos \left(\phi_{S}\right)}\left(x, z, P_{h \perp}, Q^{2}\right)=\frac{2 M}{Q} \mathcal{C}\left[-x g_{\mathrm{T}}^{q} D_{1}^{q \rightarrow h}-\frac{M_{h}}{z M} h_{1}^{q} \widetilde{E}^{q \rightarrow h}+\frac{\mathbf{p}_{T} \cdot \mathbf{k}_{T}}{2 M M_{h}} \times\right. \\
\left.\left(x e_{\mathrm{T}}^{q} H_{1}^{\perp, q \rightarrow h}-\frac{M_{h}}{z M} g_{1 \mathrm{~T}}^{q} \widetilde{D}^{\perp, q \rightarrow h}+x e_{\mathrm{T}}^{\perp, q} H_{1}^{\perp, q \rightarrow h}+\frac{M_{h}}{z M} f_{1 \mathrm{~T}}^{\perp, q} \widetilde{G}^{\perp, q \rightarrow h}\right)\right] .
\end{gathered}
$$

Also here, the two structure functions involve rather similar combinations of twist-2 and twist-3 distribution and fragmentation functions. However, the expressions simplify even more in Wandzura-Wilczek-type approximations as in addition to the interactiondependent fragmentation functions also the chiral-odd naive- $T$-odd twist-3 distributions $e_{\mathrm{T}}^{q}$ and $e_{\mathrm{T}}^{\perp, q}$ vanish, thus leaving only the contribution from the chiral-even naive- $T$-even twist-3 distributions $g_{\mathrm{T}}^{q}$ and $g_{\mathrm{T}}^{\perp, q}[64]$.

As is the case for the $F_{\mathrm{UT}}^{\sin \left(\phi_{S}\right)}$ structure function, the partial cross section given by eq. (2.16) is the only contribution to the cross section $\sigma_{\mathrm{LT}}^{h}$ that survives integration over transverse hadron momentum [8, 73, 82]:

$$
\int d^{2} \mathbf{P}_{h \perp} F_{\mathrm{LT}}^{\cos \left(\phi_{S}\right)}\left(x, z, P_{h \perp}, Q^{2}\right)=-x \frac{2 M}{Q} \sum_{q} e_{q}^{2}\left(x g_{\mathrm{T}}^{q}(x) D_{1}^{q \rightarrow h}(z)+\frac{M_{h}}{z M} h_{1}^{q}(x) \widetilde{E}^{q \rightarrow h}(z)\right) .
$$

Already in the early 1990s it was pointed out that this modulation provides collinear access to transversity in semi-inclusive deep-inelastic scattering [82], complementary to that using dihadron fragmentation [83, 84]. The challenge is to disentangle the transversity contribution from that of $g_{\mathrm{T}}^{q}$, in particular as the latter appears with the dominant $D_{1}^{q \rightarrow h}(z)$ fragmentation function.

In the inclusive limit, only the term in eq. (2.17) involving $g_{\mathrm{T}}^{q}$ can contribute. It is related to the virtual-photon-absorption asymmetries $A_{2}(x)$, used to extract information on the inclusive-DIS structure function $g_{2}(x)$ :

$$
g_{1}(x)+g_{2}(x)=\frac{1}{2} \sum_{q} e_{q}^{2} x g_{\mathrm{T}}^{q}(x)
$$

Measurements of $g_{2}$ of the proton have been published by several experiments [85-89], which could be used together with measurements of the helicity distributions to put constraints on the $g_{\mathrm{T}}^{q}$ contribution to eq. (2.17).

There is also special interest in $g_{\mathrm{T}}^{q}$ itself through its dependence on the interactiondependent function $\bar{g}_{2}$; this function is related to the transverse color Lorentz force the struck quark experiences from the spectator at the moment just after it is struck by the virtual photon [90, 91]. That is in contrast to the Sivers function, which integrates the transverse force over the length of the struck-quark's trajectory.

None of the four twist-3 Fourier amplitudes has so far been measured in semi-inclusive deep-inelastic scattering. 


\section{Measurement and analysis}

The Fourier analysis of the azimuthal transverse-target-polarization dependence of the semi-inclusive deep-inelastic scattering cross section follows closely the approach in the earlier HERMES publications on the Sivers and Collins effects for pions and charged kaons $[29,40]$. The relevant aspects of the HERMES experiment and the general analysis framework are described below, while the differences between this analysis and that of the previous publications are listed in section 3.4.

\subsection{The HERMES experiment}

The data to be presented were collected using the HERMES spectrometer [92] at the HERA lepton storage ring during the 2002-2005 running period. A longitudinally polarized positron beam (electrons in 2005) with a momentum of $27.6 \mathrm{GeV}$ traversed a transversely polarized hydrogen target.

A nuclear-polarized pure-hydrogen gas target [93] internal to the HERA lepton storage ring was used, providing highly polarized target samples without dilution from unpolarized target material or background arising from unwanted scattering from the target-material container. Furthermore, this technique included rapid reversals of target-spin orientations, with the sign randomly chosen at 1-3 min time intervals. This provided a substantial reduction of time-dependent systematic uncertainties. For the 2002-2005 running, an average degree of polarization, perpendicular to the lepton-beam direction, of $0.725 \pm 0.053$ was achieved.

The $27.6 \mathrm{GeV}$ electron or positron beam of HERA became self-polarized in the transverse direction due to a tiny spin-flip asymmetry in the emission of synchrotron radiation (Sokolov-Ternov effect) [94]. Longitudinal beam polarization was then obtained through spin rotators installed up- and down-stream of the HERMES interaction region. Every few months, the longitudinal beam polarization was reversed to allow balancing of data for the two helicity states. For the data presented, the typical beam-polarization values are between $30 \%$ and $40 \%$ in magnitude, with a negligible net polarization when averaged over the whole data-taking period.

Scattered leptons and charged hadrons produced in the forward direction were detected within an angular acceptance of about $\pm 170 \mathrm{mrad}$ horizontally and about $\pm(40-140) \mathrm{mrad}$ vertically. Charged-particle tracks were reconstructed using a set of drift chambers in front of and behind the $1.6 \mathrm{Tm}$ dipole magnet and corrected for the bending within the target magnetic field, resulting in an average momentum and angular resolution of about $1.5 \%$ each.

The particle-identification system consisted of a dual-radiator ring-imaging Cherenkov (RICH) detector, a transition-radiation detector, a pre-shower scintillation counter and an electromagnetic calorimeter. The PID system provided a lepton identification with an efficiency of $98 \%$ and a hadron contamination of less than $1 \%$. In the momentum range $2 \mathrm{GeV}<\left|\mathbf{P}_{h}\right|<15 \mathrm{GeV}$, charged pions, kaons, and protons ${ }^{11}$ are identified by using the

\footnotetext{
${ }^{11}$ The momentum range for (anti)protons is later restricted to $4 \mathrm{GeV}<\left|\mathbf{P}_{h}\right|<15 \mathrm{GeV}$ in order to avoid the low-momentum region of large meson contamination due to inefficiencies of the RICH.
} 


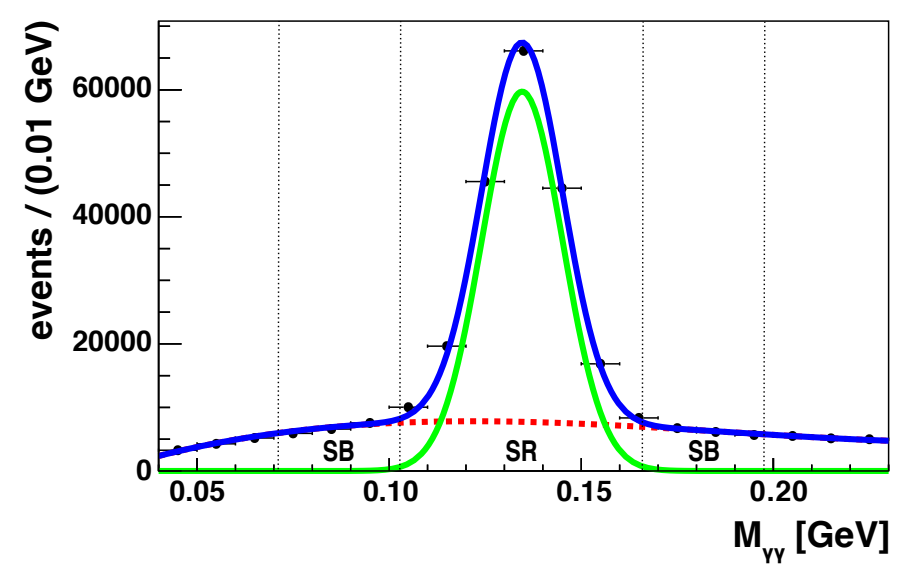

Figure 2. The two-photon invariant-mass distribution in the region of the $\pi^{0}$ mass for the overall data sample. The sum (blue line) of a Gaussian for the $\pi^{0}$ signal (green line) and a third-order Chebyshev polynomial for the combinatorial background (red dashed curve) are fit to data. The signal range used in the analysis, indicated as "SR", spans a $\pm 3 \sigma$ region around the $\pi^{0}$ peak position. Events for the background subtraction are selected from the sidebands denoted by "SB". The signal-region and sideband boundaries are indicated by vertical dotted lines.

RICH detector [95], for which a hadron-identification algorithm is applied that takes into account the event topology [32].

The electromagnetic calorimeter and the pre-shower scintillation counter were also employed in detecting photons with an energy above $1 \mathrm{GeV}$, which are used here in reconstructing neutral pions. Unaffected by the magnetic fields of both the target and the spectrometer magnet, photons were accepted in the horizontal and vertical angular ranges of $\pm 175 \mathrm{mrad}$ and $\pm(43-147) \mathrm{mrad}$, respectively.

Neutral pions are reconstructed using their dominant decay into two photons. The decay length of the $\pi^{0}$ is negligible compared to the resolution of the spectrometer, hence the decay vertex is assumed to coincide with the lepton-scattering vertex. The photon pairs produced within the acceptance of the spectrometer generate electromagnetic showers in the calorimeter, a fraction of the photons starting a shower already in the lead sheet of the pre-shower detector, which is taken into account in the energy determination of the photon. For each deep-inelastic scattering event with more than one photon detected in the calorimeter, the invariant mass of all possible photon-pair combinations is calculated under the assumption that the photon-pair originated from the lepton-scattering vertex. The resulting two-photon invariant-mass distribution for the overall data sample is shown in figure 2. In each kinematic bin, the signal range is determined by a $\pm 3 \sigma$ window around the $\pi^{0}$ peak position of the invariant-mass distribution, where $1 \sigma$ reflects the energy resolution of the calorimeter. For the subtraction of the combinatorial background, events from sidebands to the left and right of the peak were used, appropriately weighted to reflect the amount of background in the signal region. 


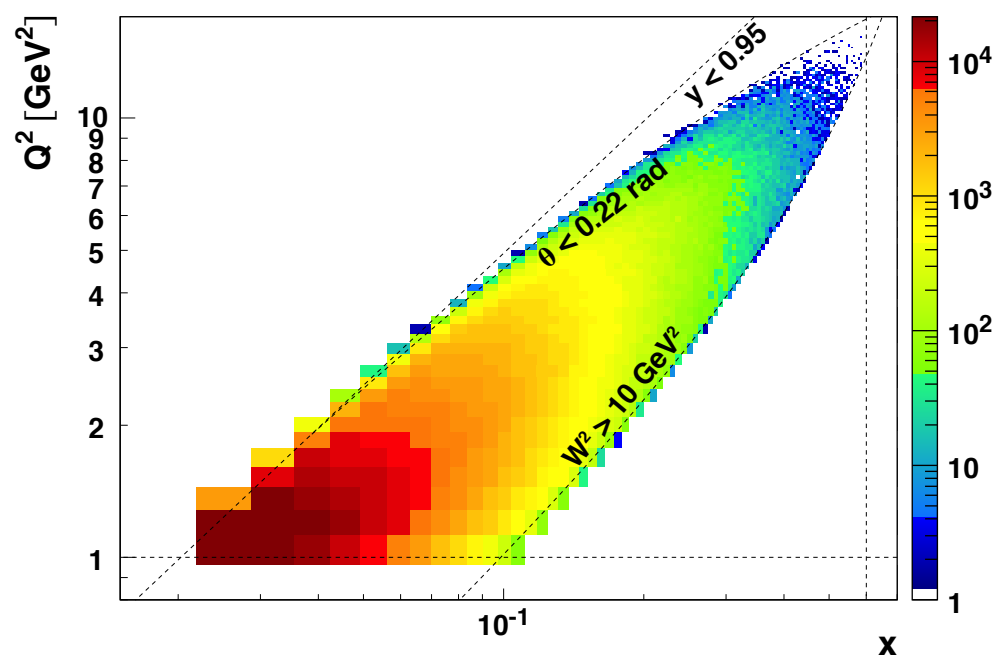

Figure 3. Event distribution in the kinematic space in $\left(x, Q^{2}\right)$, including the various boundaries arising from constraints on $x, y, Q^{2}, W^{2}$, and the upper reach in the lepton scattering angle $\theta$.

\subsection{Data selection}

Identified leptons were subject to various kinematic requirements in order to select a "deepinelastic scattering" sample:

(i) Final-state electrons and positrons are kept (including leptons with charge opposite to the beam leptons) in order to apply a correction for background contributions from pair-production processes.

(ii) The hard scattering scale of the deep-inelastic scattering process is constrained to $Q^{2}>1 \mathrm{GeV}^{2}$.

(iii) Based on the chosen scale and the limited angular acceptance of the spectrometer, the Bjorken scaling variable is required to be in the range $0.023<x<0.6$.

(iv) Scattering events originating from the excitation of nucleon resonances and their subsequent strong decays are excluded by the requirement $W^{2}>10 \mathrm{GeV}^{2}$ on the squared invariant mass of the photon-nucleon system $W^{2} \equiv(q+P)^{2}$.

(v) The upper limit on $y$ is implied only by the calorimeter threshold of $1.4 \mathrm{GeV}(y<0.95)$. The lower limit on $y$ is dictated by the $W^{2}$ constraint, resulting in a minimum $y$ of 0.18 , which increases with $x$. No further restrictions are applied as they would have enhanced the strong correlation between the scaling variables $x$ and $Q^{2}$.

The resulting kinematic phase-space in the $x-Q^{2}$ plane is shown in figure 3 , where also the constraints on $x, y, Q^{2}, W^{2}$, and the upper reach in the lepton scattering angle are indicated. The strong correlation between $x$ and $Q^{2}$ is apparent.

The "semi-inclusive deep-inelastic scattering" sample fulfills in addition the following criteria: 


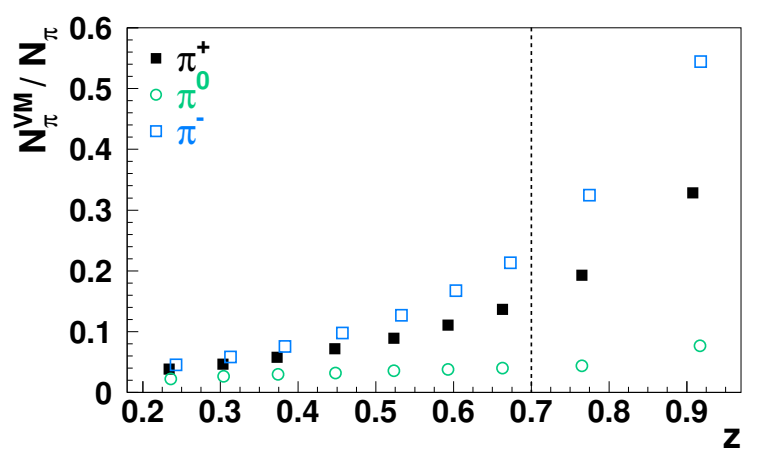

Figure 4. The simulated fraction of pions originating from diffractive vector-meson production and decay is shown as a function of $z$. (The open squares indicating $\pi^{-}$are slightly shifted horizontally). The contributions are simulated by a version of PYTHIA6.2 [96, 97] tuned for HERMES kinematics. By limiting $z$ to $z<0.7$, a kinematic region is probed where the vector-meson contribution to the electroproduction of pions is suppressed, in particular for charged pions. For charged kaons, the contribution from $\phi$ decay is at maximum $10 \%$ [98].

(i) All identified hadrons are selected (and not only the leading hadron, i.e., the one with the highest momentum in the event).

(ii) A lower limit $z>0.2$ is applied to suppress contributions from the target fragmentation region.

(iii) An upper limit $z<0.7$ is generally applied to suppress contributions from hadrons originating from the decay of diffractively produced vector-mesons. As shown in figure 4, contributions due to exclusive channels (in particular for charged pions) become sizable at large $z$. However, when looking at only the one-dimensional $z$ dependence of the azimuthal asymmetries, this requirement is lifted and instead an upper limit of 1.2 (driven by the detector resolution) is imposed, in order to probe this "semi-exclusive" transition region. The resulting yield distributions for the positively charged hadrons are shown in figure 5 (left). The shift towards higher $z$ in the distribution of protons mainly results from the larger hadron mass and the $4 \mathrm{GeV}$ minimum-momentum requirement (compared to $2 \mathrm{GeV}$ for charged mesons).

(iv) The formalism of TMD factorization involves one hard scale, $Q^{2}$, and transverse momenta that are small in comparison. While no lower limit on $P_{h \perp}$ is imposed, an upper limit of $P_{h \perp}<2 \mathrm{GeV}$ is applied in this analysis (cf. figure 5, right). On average, the constraint $P_{h \perp}^{2} \ll Q^{2}$ is fulfilled for most deep-inelastic scattering events (cf. figure 6), while the stricter constraint $P_{h \perp}^{2} \ll z^{2} Q^{2}$ is often violated at large $P_{h \perp}$ in the kinematic region of low $x$ (which corresponds to low $Q^{2}$ ) and low $z .^{12}$

Recently, separation of current and target fragmentation has been revisited for semiinclusive deep-inelastic scattering involving transverse momentum [99]. In particular, low- $z$

\footnotetext{
${ }^{12} \mathrm{~A}$ more detailed discussion is presented in appendix B, including further distributions, e.g., for the more critical region of low $z$ and $Q^{2}$.
} 

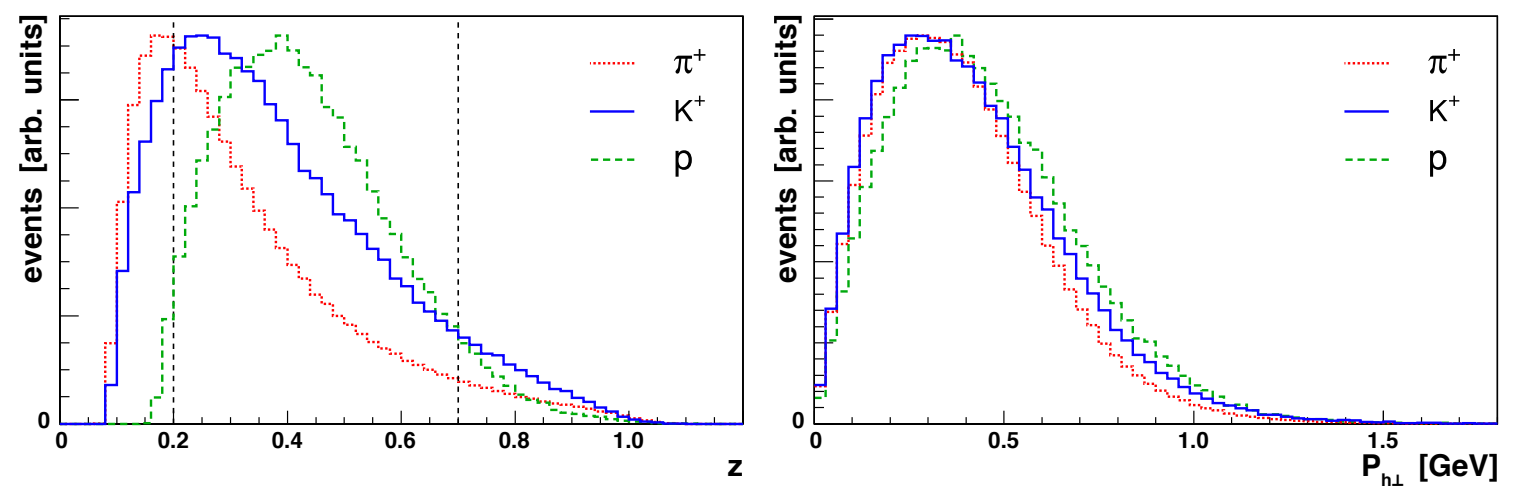

Figure 5. Shape comparison of arbitrarily normalized $\pi^{+}$(red dotted line), $K^{+}$(blue line), and proton (green dashed line) yield distributions in the hadron variables $z$ (left) and $P_{h \perp}$ (right). The region between the two vertical dashed lines indicates the range in $z$ used for the semi-inclusive DIS sample, while events in the extended range $0.7<z<1.2$ are analyzed only in the one-dimensional $z$ binning.

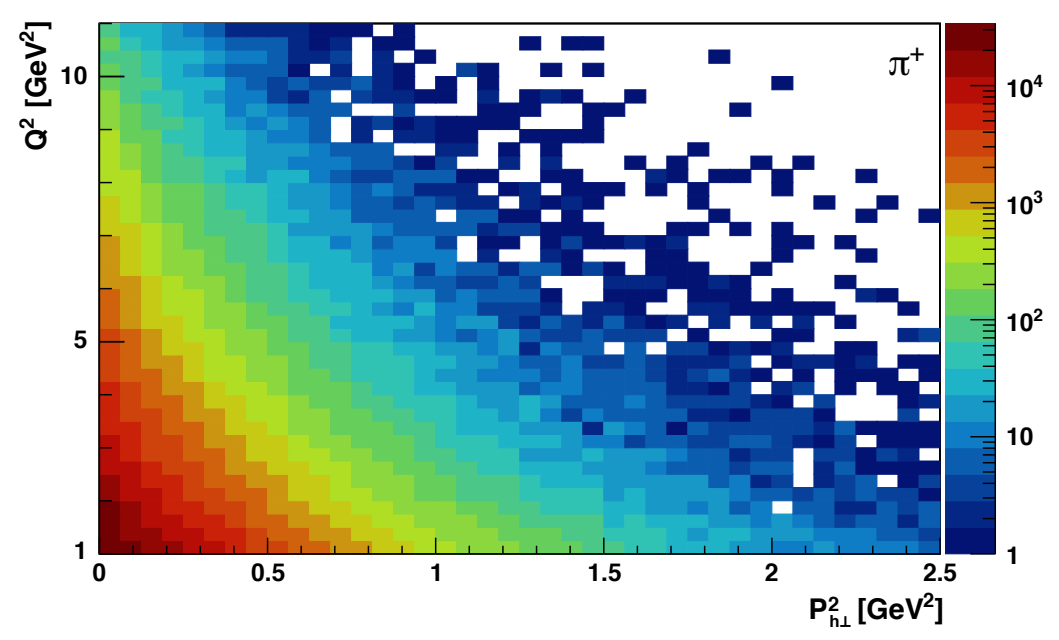

Figure 6. Distribution in $Q^{2}$ versus $P_{h \perp}^{2}$ of the semi-inclusive $\pi^{+}$yield.

hadrons with large transverse momentum might originate from the remnants of the target and not from the fragmentation of the struck quark [100, 101], the region that is described here in terms of TMD distribution and fragmentation functions. While no general recipe, e.g., a quantitative limit on kinematic variables, is available, it appears appropriate to provide additional information about the kinematic distributions in this measurement. For this it is useful to introduce both Feynman- $x, x_{F}$, the ratio of the longitudinal hadron momentum $P_{h \|}^{\mathrm{CM}}$ along the virtual-photon direction to its maximum possible value in the virtual-photon-nucleon center-of-mass system (CM), and rapidity,

$$
y_{h} \equiv \frac{1}{2} \ln \frac{P_{h}^{+}}{P_{h}^{-}}
$$

where $P_{h}^{ \pm}$are the \pm light-cone momenta, i.e., $E_{h}^{\mathrm{CM}} \pm P_{h \|}^{\mathrm{CM}}$, of the hadron in the virtual- 


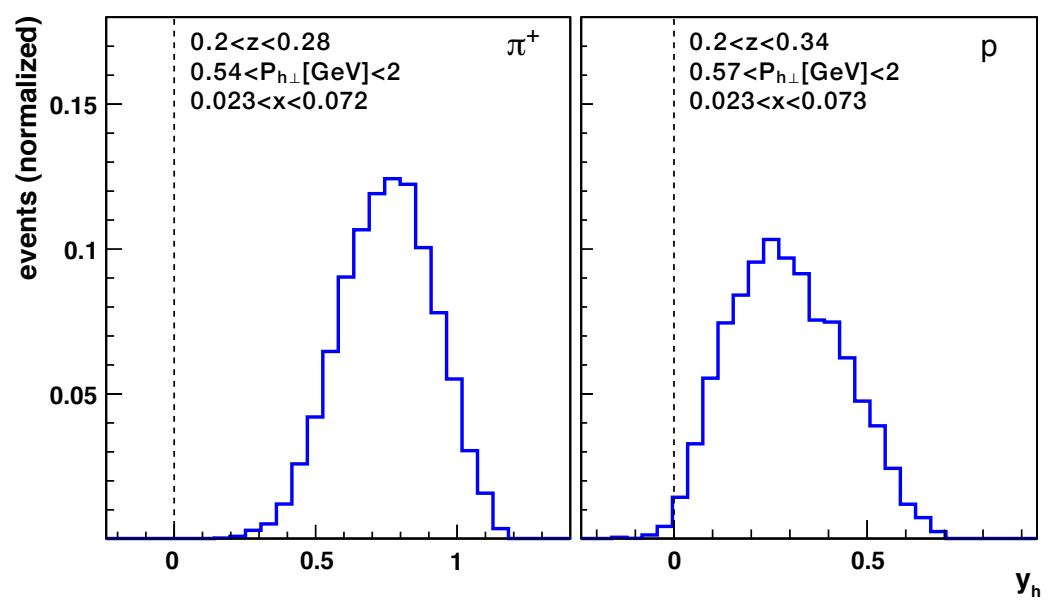

Figure 7. Rapidity distributions for $\pi^{+}$(left) and protons (right) in the kinematic region indicated. (Distributions are normalized to unity.)

\begin{tabular}{|c|c|c|c|}
\hline \multirow[t]{4}{*}{ Scattered lepton: } & & $Q^{2}$ & $>1 \mathrm{GeV}^{2}$ \\
\hline & & $W^{2}$ & $>10 \mathrm{GeV}^{2}$ \\
\hline & $0.023<$ & $x$ & $<0.6$ \\
\hline & $0.1<$ & $y$ & $<0.95$ \\
\hline \multirow[t]{5}{*}{ Detected hadrons: } & $2 \mathrm{GeV}<$ & $\left|\mathbf{P}_{h}\right|$ & charged mesons \\
\hline & $4 \mathrm{GeV}<$ & $\left|\mathbf{P}_{h}\right|$ & $<15 \mathrm{GeV} \quad$ (anti)protons \\
\hline & & $\left|\mathbf{P}_{h}\right|$ & $>2 \mathrm{GeV} \quad$ neutral pions \\
\hline & & $P_{h \perp}$ & $<2 \mathrm{GeV}$ \\
\hline & $0.2<$ & $z$ & $<0.7$ (1.2 for the "semi-exclusive" region) \\
\hline
\end{tabular}

Table 3. Restrictions on selected kinematics variables. The upper limit on $z$ of 1.2 applies only to the analysis of the $z$ dependence.

\begin{tabular}{|cccccccc|}
\hline & $\pi^{+}$ & $\pi^{0}$ & $\pi^{-}$ & $K^{+}$ & $K^{-}$ & $p$ & $\bar{p}$ \\
\hline $0.2<z<0.7$ & $755 \mathrm{k}$ & $158 \mathrm{k}$ & $543 \mathrm{k}$ & $136 \mathrm{k}$ & $57 \mathrm{k}$ & $94 \mathrm{k}$ & $14 \mathrm{k}$ \\
$0.7<z<1.2$ & $68 \mathrm{k}$ & $10 \mathrm{k}$ & $40 \mathrm{k}$ & $14 \mathrm{k}$ & $1 \mathrm{k}$ & $6 \mathrm{k}$ & $<1 \mathrm{k}$ \\
\hline
\end{tabular}

Table 4. Hadron yields for the semi-inclusive DIS range and the high- $z$ region.

photon-nucleon center-of-mass system. Both are measures of the "forwardness" of the hadron in that system. Positive values of $x_{F}$ and $y_{h}$ are more likely associated with hadrons produced from the struck quark, while negative values point at target fragmentation. As an example, the rapidity distributions for $\pi^{+}$and protons are shown in figure 7 for a specific kinematic bin of small $z$ and large $P_{h \perp}$. Even though proton production is more susceptible to contributions from target fragmentation, the proton's rapidity remains, like that of pions, mainly positive. Further discussion including more distributions can be found in appendix B.

The criteria for the selection of scattered leptons and of hadrons detected in coincidence are summarized in table 3 . They have been chosen to ensure a good semi-inclusive 


\begin{tabular}{|ccc|}
\hline$x$ bins & $z$ bins & $P_{h \perp}$ bins \\
\hline$] 0.023 ; 0.072]$ & ] $0.20 ; 0.28]$ & ] $0.00 \mathrm{GeV} ; 0.23 \mathrm{GeV}]$ \\
] $0.072 ; 0.098]$ & ] $0.28 ; 0.37]$ & ] $0.23 \mathrm{GeV} ; 0.36 \mathrm{GeV}]$ \\
] $0.098 ; 0.138]$ & ] $0.37 ; 0.49]$ & ] $0.36 \mathrm{GeV} ; 0.54 \mathrm{GeV}]$ \\
] $0.138 ; 0.600]$ & ] $0.49 ; 0.70]$ & ] $0.54 \mathrm{GeV} ; 2.00 \mathrm{GeV}]$ \\
\hline
\end{tabular}

Table 5. Definition of the three-dimensional binning for charged mesons: the first, second, and third columns list the limits in the kinematic variables $x, z$, and $P_{h \perp}$, respectively.

\begin{tabular}{|ccc|}
\hline$x$ bins & $z$ bins & $P_{h \perp}$ bins \\
\hline$] 0.023 ; 0.073]$ & ] $0.20 ; 0.34]$ & ] $0.00 \mathrm{GeV} ; 0.24 \mathrm{GeV}]$ \\
] $0.073 ; 0.107]$ & ] $0.34 ; 0.43]$ & ] $0.24 \mathrm{GeV} ; 0.40 \mathrm{GeV}]$ \\
] $0.107 ; 0.157]$ & ] $0.43 ; 0.52]$ & ] $0.40 \mathrm{GeV} ; 0.57 \mathrm{GeV}]$ \\
] $0.157 ; 0.600]$ & ] $0.52 ; 0.70]$ & ] $0.57 \mathrm{GeV} ; 2.00 \mathrm{GeV}]$ \\
\hline
\end{tabular}

Table 6. Definition of the three-dimensional binning for protons: the first, second, and third columns list the limits in the kinematic variables $x, z$, and $P_{h \perp}$, respectively.

deep-inelastic scattering measurement, e.g., adequate detector resolutions and minimal backgrounds, but have not be tuned to the requirements of current TMD factorization only. The data are thus sensitive to kinematic regions in semi-inclusive deep-inelastic scattering, including various transition regions that are under theoretical investigation. The final number of hadrons after the application of all selection criteria is provided in table 4 for both the semi-inclusive range of $0.2<z<0.7$ and the extended range of $0.7<z<1.2$.

\subsection{The extraction of the asymmetry amplitudes}

Signals for TMDs are extracted using an unbinned maximum-likelihood fit to their distinctive signatures in the azimuthal angles $\phi$ and $\phi_{S}$. The extracted Fourier components are studied as a function of the kinematic variables $x, z$, and $P_{h \perp}$. As the three-dimensional dependence of the asymmetry amplitudes does not factorize a priori, the primary results of this analysis are provided in a three-dimensional binning in those kinematic variables. Binning the data also in $Q^{2}$ (or alternatively $y$ ) is not applicable by lack of statistical precision. The bin sizes and boundaries are optimized for the various hadrons in order to have results in all bins. This results in two sets of $4 \times 4 \times 4$ grids with a total of 64 bins each for charged mesons and for protons (see tables 5 and 6 , respectively). The yields for neutral pions and for antiprotons are insufficient for using such three-dimensional binning.

In addition to the full information given in the three-dimensional representations, results for one-dimensional projections are provided, for which the data are subdivided into seven bins in either $x, z$, or $P_{h \perp}$. This allows presenting results also for neutral pions and antiprotons, but also a much faster evaluation of key characteristics of the results. Furthermore, the range in $z$ is extended by further three bins to include also the high- $z$ "semi-exclusive" region. As before, the binning differs slightly for mesons and (anti)protons due to the different kinematic requirements and underlying distributions. The resulting bin boundaries are given for mesons in table 7 and for (anti)protons in table 8 . 


\begin{tabular}{|cccc|}
\hline Bin & $x$ dependence & $z$ dependence & $P_{h \perp}$ dependence \\
\hline 1 & ] $0.023 ; 0.046]$ & ] $0.20 ; 0.26]$ & ] $0.00 \mathrm{GeV} ; 0.17 \mathrm{GeV}]$ \\
2 & ] $0.046 ; 0.067]$ & ] $0.26 ; 0.32]$ & ] $0.17 \mathrm{GeV} ; 0.25 \mathrm{GeV}]$ \\
3 & ] $0.067 ; 0.082]$ & ] $0.32 ; 0.38]$ & ] $0.25 \mathrm{GeV} ; 0.31 \mathrm{GeV}]$ \\
4 & ] $0.082 ; 0.105]$ & ] $0.38 ; 0.45]$ & ] $0.31 \mathrm{GeV} ; 0.38 \mathrm{GeV}]$ \\
5 & ] $0.105 ; 0.134]$ & ] $0.45 ; 0.52]$ & ] $0.38 \mathrm{GeV} ; 0.52 \mathrm{GeV}]$ \\
6 & ] $0.134 ; 0.186]$ & ] $0.52 ; 0.60]$ & ] $0.52 \mathrm{GeV} ; 0.69 \mathrm{GeV}]$ \\
7 & ] $0.186 ; 0.600]$ & ] $0.60 ; 0.70]$ & ] $0.69 \mathrm{GeV} ; 2.00 \mathrm{GeV}]$ \\
8 & & ] $0.70 ; 0.76]$ & \\
9 & & ] $0.76 ; 0.84]$ & \\
10 & & ] $0.84 ; 1.20]$ & \\
\hline
\end{tabular}

Table 7. Definition of the one-dimensional binning for mesons: the first column lists the bin number; the second, third, and fourth columns give the corresponding limits in the kinematic variables $x, z$, and $P_{h \perp}$, respectively.

\begin{tabular}{|cccc|}
\hline Bin & $x$ dependence & $z$ dependence & $P_{h \perp}$ dependence \\
\hline 1 & ] $0.023 ; 0.040]$ & ] $0.20 ; 0.27]$ & ] $0.00 \mathrm{GeV} ; 0.23 \mathrm{GeV}]$ \\
2 & ] $0.040 ; 0.057]$ & ] $0.27 ; 0.34]$ & ] $0.23 \mathrm{GeV} ; 0.34 \mathrm{GeV}]$ \\
3 & ] $0.057 ; 0.075]$ & ] $0.34 ; 0.41]$ & ] $0.34 \mathrm{GeV} ; 0.43 \mathrm{GeV}]$ \\
4 & ] $0.075 ; 0.098]$ & ] $0.41 ; 0.47]$ & ] $0.43 \mathrm{GeV} ; 0.52 \mathrm{GeV}]$ \\
5 & ] $0.098 ; 0.136]$ & ] $0.47 ; 0.53]$ & ] $0.52 \mathrm{GeV} ; 0.62 \mathrm{GeV}]$ \\
6 & ] $0.136 ; 0.185]$ & ] $0.53 ; 0.61]$ & ] $0.62 \mathrm{GeV} ; 0.74 \mathrm{GeV}]$ \\
7 & ] $0.185 ; 0.600]$ & ] $0.61 ; 0.70]$ & ] $0.74 \mathrm{GeV} ; 2.00 \mathrm{GeV}]$ \\
8 & & ] $0.70 ; 0.78]$ & \\
9 & & ] $0.78 ; 0.88]$ & \\
10 & & ] $0.88 ; 1.20]$ & \\
\hline
\end{tabular}

Table 8. Definition of the one-dimensional binning for (anti)protons: the first column lists the bin number; the second, third, and fourth columns give the corresponding limits in the kinematic variables $x, z$, and $P_{h \perp}$, respectively.

\subsubsection{The choice of the probability-density function}

Ideally, the various structure functions of the semi-inclusive cross section (2.1) are extracted directly. However, experimentally such an extraction would require precision knowledge of the luminosity and all the instrumental effects, e.g., efficiencies and geometrical acceptance. Instead, in the measurement reported here the rapid spin reversal of the target protons is exploited to effectively extract spin asymmetries. While avoiding many of the experimental uncertainties, theoretical uncertainties arise in the interpretation of the results as they constitute relative quantities by normalizing the polarization-dependent structure functions to the polarization-averaged and $\phi$-integrated cross section, which is proportional to $F_{\mathrm{UU}, \mathrm{T}}^{h}+\epsilon F_{\mathrm{UU}, \mathrm{L}}^{h}$. The detailed knowledge of the latter is still limited, in particular the transverse-momentum dependence, but also the contribution from longitudinal photons. In the case of inclusive deep-inelastic scattering at HERMES kinematics, the contribution 
from longitudinal photons can reach values of up to $30 \%$ compared to the one from transverse photons (used to interpret the structure functions in the parton model at leading order in $\left.\alpha_{S}\right)$.

An experimental limitation is the inability to polarize the target on an event-by-event basis with respect to the virtual-photon direction. The latter is used in eq. (2.1) as a reference axis because it is a more convenient and natural choice for the decomposition. In contrast, in an actual experiment, target-polarization states are chosen with respect to the incident-lepton direction. The coordinate transformation from the lepton-beam system to the virtual-photon system and its effects are worked out in ref. [102]. It involves the usually small polar angle $\theta_{\gamma^{*}}$ between the incident-lepton and virtual-photon three-momenta. As discussed in more detail in appendix $\mathrm{A}$, the observable azimuthal modulations, labeled henceforth by $\perp(\|)$ instead of $\mathrm{T}(\mathrm{L})$ for the transverse (longitudinal) target-polarization component, are in general a mixture of contributions from the target-polarization terms labeled with $\mathrm{T}$ and $\mathrm{L}$ in eq. (2.1). Moreover, the Fourier decomposition of the azimuthal distribution for the $\perp(\|)$ configuration includes additional terms not present in eq. (2.1). In particular, for $\perp$ target polarization an additional $\sin \left(2 \phi+\phi_{S}\right)\left[\cos \left(\phi+\phi_{S}\right)\right]$ modulation is possible when the lepton beam is unpolarized [longitudinally polarized]. The number of azimuthal modulations for hadron leptoproduction on a target polarized perpendicular to the direction of the incident lepton are thus ten: six single-spin and four doublespin asymmetries. Of those, three $\left[\sin \phi_{S}, \sin \left(3 \phi-\phi_{S}\right)\right.$, and $\left.\cos \left(2 \phi-\phi_{S}\right)\right]$ arise genuinely from transverse target polarization, five $\left[\sin \left(\phi+\phi_{S}\right), \sin \left(\phi-\phi_{S}\right), \sin \left(2 \phi-\phi_{S}\right), \cos \phi_{S}\right.$, and $\left.\cos \left(\phi-\phi_{S}\right)\right]$ are dominantly transverse-polarization effects with a small admixture from longitudinal target polarization, and the remaining two are genuine contributions from the small but non-vanishing longitudinal target-polarization component.

In this measurement, a maximum-likelihood fit is employed that incorporates the reversal of both the beam and target polarization in the probability density function. The probability density for the combined Fourier analysis of single-spin and double-spin azimuthal asymmetries is modeled according to the cross-section contributions $\sigma_{\mathrm{U} \perp}^{h}$ and $\sigma_{\mathrm{L} \perp}^{h}$. As such it includes a total of ten modulations: the six sine modulations of the cross section $\sigma_{\mathrm{U} \perp}^{h}$ and, when including the longitudinal lepton-beam polarization, four cosine modulations.

Another choice has to be made concerning which kinematic terms of the cross section to include as part of the parameters to be fit. Two possibilities are presented here: the cross-section asymmetries (CSA), which involve - up to prefactors common to all crosssection terms - the entire Fourier amplitude of each cross-section modulation, e.g., also the $\epsilon$-dependent kinematic prefactors. In contrast, the structure-function asymmetries (SFA) are to first approximation ratios of only the structure functions discussed in more detail in section 2.2, obtained by including explicitly the $\epsilon$-dependent kinematic prefactors in the likelihood function separated from the fit parameters. A compelling advantage of the latter asymmetries is their simple interpretation. The strongly experiment-dependent kinematic prefactors contain little additional information and cloud direct comparisons to results from different experiments. The advantages of the former include the possibility to correct in a straightforward way for the contributions from the longitudinal target-polarization 
component (cf. appendix A), ${ }^{13}$ and the independence of the analysis from the particular assumptions made in the expansion of the modulations in terms of structure functions. The primary results presented here will be the structure-function asymmetries.

In the case of perfect acceptance in the azimuthal angles, each Fourier amplitude could be extracted separately due to orthogonality of the azimuthal modulations. However, under realistic experimental conditions cross-contamination may occur. Hence, both the single-spin and double-spin Fourier amplitudes are extracted simultaneously. The corresponding probability-density function for the Fourier decomposition of the cross section (CSA decomposition) is then defined as

$$
\begin{aligned}
\mathbb{P}\left(x, z, P_{h \perp}, \phi, \phi_{S}, P_{l}, S_{\perp}: 2\left\langle\sin \left(\phi-\phi_{S}\right)\right\rangle_{\mathrm{U} \perp}^{h}, \ldots 2\left\langle\cos \left(\phi+\phi_{S}\right)\right\rangle_{\mathrm{L} \perp}^{h}\right) \\
=\left[1+S_{\perp}\left(2\left\langle\sin \left(\phi-\phi_{S}\right)\right\rangle_{\mathrm{U} \perp}^{h} \sin \left(\phi-\phi_{S}\right)+2\left\langle\sin \left(\phi+\phi_{S}\right)\right\rangle_{\mathrm{U} \perp}^{h} \sin \left(\phi+\phi_{S}\right)+\right.\right. \\
2\left\langle\sin \left(3 \phi-\phi_{S}\right)\right\rangle_{\mathrm{U} \perp}^{h} \sin \left(3 \phi-\phi_{S}\right)+2\left\langle\sin \left(\phi_{S}\right)\right\rangle_{\mathrm{U} \perp}^{h} \sin \left(\phi_{S}\right)+ \\
\left.2\left\langle\sin \left(2 \phi-\phi_{S}\right)\right\rangle_{\mathrm{U} \perp}^{h} \sin \left(2 \phi-\phi_{S}\right)+2\left\langle\sin \left(2 \phi+\phi_{S}\right)\right\rangle_{\mathrm{U} \perp}^{h} \sin \left(2 \phi+\phi_{S}\right)\right) \\
+P_{l} S_{\perp}\left(2\left\langle\cos \left(\phi-\phi_{S}\right)\right\rangle_{\mathrm{L} \perp}^{h} \cos \left(\phi-\phi_{S}\right)+2\left\langle\cos \left(\phi_{S}\right)\right\rangle_{\mathrm{L} \perp}^{h} \cos \left(\phi_{S}\right)+\right. \\
\left.\left.2\left\langle\cos \left(2 \phi-\phi_{S}\right)\right\rangle_{\mathrm{L} \perp}^{h} \cos \left(2 \phi-\phi_{S}\right)+2\left\langle\cos \left(\phi+\phi_{S}\right)\right\rangle_{\mathrm{L} \perp}^{h} \cos \left(\phi+\phi_{S}\right)\right)\right]^{w},
\end{aligned}
$$

where $P_{l}$ and $S_{\perp}$ represent the degree of longitudinal beam polarization and target polarization perpendicular to the lepton beam, respectively, and $w$ is an event weight further detailed below. The Fourier amplitudes $2\left\langle\sin \left(\phi-\phi_{S}\right)\right\rangle_{\mathrm{U} \perp}^{h}, \ldots 2\left\langle\cos \left(\phi+\phi_{S}\right)\right\rangle_{\mathrm{L} \perp}^{h}$ appearing as parameters in eq. (3.2) are the cross-section asymmetry amplitudes to be fit to the data. Likewise, the probability-density function for the SFA amplitudes reads

$$
\begin{aligned}
& \mathbb{P}\left(x, z, \epsilon, P_{h \perp}, \phi, \phi_{S}, P_{l}, S_{\perp}: 2\left\langle\sin \left(\phi-\phi_{S}\right)\right\rangle_{\mathrm{U} \perp}^{h}, \ldots 2\left\langle\cos \left(\phi+\phi_{S}\right) / \sqrt{2 \epsilon(1-\epsilon)}\right\rangle_{\mathrm{L} \perp}^{h}\right) \\
&=\left[1+S_{\perp}\left(2\left\langle\sin \left(\phi-\phi_{S}\right)\right\rangle_{\mathrm{U} \perp}^{h} \sin \left(\phi-\phi_{S}\right)+\epsilon 2\left\langle\sin \left(\phi+\phi_{S}\right) / \epsilon\right\rangle_{\mathrm{U} \perp}^{h} \sin \left(\phi+\phi_{S}\right)+\right.\right. \\
& \epsilon 2\left\langle\sin \left(3 \phi-\phi_{S}\right) / \epsilon\right\rangle_{\mathrm{U} \perp}^{h} \sin \left(3 \phi-\phi_{S}\right)+ \\
& \sqrt{2 \epsilon(1+\epsilon)} 2\left\langle\sin \left(\phi_{S}\right) / \sqrt{2 \epsilon(1+\epsilon)}\right\rangle_{\mathrm{U} \perp}^{h} \sin \left(\phi_{S}\right)+ \\
& \sqrt{2 \epsilon(1+\epsilon)} 2\left\langle\sin \left(2 \phi-\phi_{S}\right) / \sqrt{2 \epsilon(1+\epsilon)}\right\rangle_{\mathrm{U} \perp}^{h} \sin \left(2 \phi-\phi_{S}\right)+ \\
& \epsilon\left.2\left\langle\sin \left(2 \phi+\phi_{S}\right) / \epsilon\right\rangle_{\mathrm{U} \perp}^{h} \sin \left(2 \phi+\phi_{S}\right)\right) \\
&+P_{l} S_{\perp}\left(\sqrt{1-\epsilon^{2}} 2\left\langle\cos \left(\phi-\phi_{S}\right) / \sqrt{1-\epsilon^{2}}\right\rangle_{\mathrm{L} \perp}^{h} \cos \left(\phi-\phi_{S}\right)+\right. \\
& \sqrt{2 \epsilon(1-\epsilon)} 2\left\langle\cos \left(\phi_{S}\right) / \sqrt{2 \epsilon(1-\epsilon)}\right\rangle_{\mathrm{L} \perp}^{h} \cos \left(\phi_{S}\right)+ \\
& \sqrt{2 \epsilon(1-\epsilon)} 2\left\langle\cos \left(2 \phi-\phi_{S}\right) / \sqrt{2 \epsilon(1-\epsilon)}\right\rangle_{\mathrm{L} \perp}^{h} \cos \left(2 \phi-\phi_{S}\right)+ \\
&\left.\left.\sqrt{2 \epsilon(1-\epsilon)} 2\left\langle\cos \left(\phi+\phi_{S}\right) / \sqrt{2 \epsilon(1-\epsilon)}\right\rangle_{\mathrm{L} \perp}^{h} \cos \left(\phi+\phi_{S}\right)\right)\right]^{w} .
\end{aligned}
$$

\footnotetext{
${ }^{13}$ For example, the contributions from the transverse and longitudinal components of the target polarization may have different kinematic prefactors, which cannot be taken into account in the fit of structurefunction asymmetries.
} 
Charged-hadron [pion, kaon, and (anti-)proton] weights are assigned to each hadron track selected to account for the efficiency of the RICH detector and the contamination of the pion, kaon, and proton identification. When the charge of the scattered lepton does not correspond to the charge of the incoming beam leptons, the weights are multiplied by -1 in order to subtract the background arising from the pair-production process. In a similar way, combinatorial background in the $\pi^{0}$ signal region of the two-photon invariantmass spectrum is subtracted using events from the sidebands (cf. figure 2) and assigning a negative weight equal to $-R$, where the ratio $R$ is the relative population of combinatorial background in the signal region and the sidebands, as given by the invariant-mass fit.

As the sum over all weights does not coincide with the number of hadrons detected, i.e.,

$$
\sum_{i=1}^{N^{h}} w_{i} \neq N^{h}
$$

the statistical uncertainties of the asymmetry amplitudes extracted have to be corrected for the event weighting. The covariance matrix $C$, obtained in the maximum-likelihood fit, is corrected by the covariance matrix $K$ that is obtained in a maximum-likelihood fit to the same data but weighting the events with $w_{i}^{2}$ instead of $w_{i}$. The statistical uncertainties are then evaluated from the corrected covariance matrix [103]

$$
C^{\prime}=C K^{-1} C \text {. }
$$

In the likelihood formalism, not only the target polarization but also the beam polarization is applied on event level, i.e., for each identified hadron of a given semi-inclusive deep-inelastic scattering event, the actual beam and target polarization values of that event are used in the likelihood function.

The normalization of the probability density function is not required as in the data set selected the net target polarization is found to be negligible. Thus, the normalization integral is independent of the asymmetry amplitudes extracted and cannot influence the shape of the likelihood dependence on the azimuthal amplitudes.

The CSA amplitudes are then extracted from the semi-inclusive deep-inelastic scattering events by minimizing

$$
-\ln \mathbb{L}=-\sum_{i=1}^{N_{h}} w_{i} \ln \mathbb{P}\left(x_{i}, z_{i}, P_{h \perp, i}, \phi_{i}, \phi_{S, i}, P_{l, i}, S_{\perp, i}: 2\left\langle\sin \left(\phi-\phi_{S}\right)\right\rangle_{\mathrm{U} \perp}^{h}, \ldots\right)
$$

using eq. (3.2) for $\mathbb{P}(\ldots)$. In a similar fashion, eq. (3.3) is used to extract the SFA amplitudes, including now also the event-wise value of the photon-polarization parameter $\epsilon$.

Comparing eqs. (3.2) and (3.3) with eq. (2.1), it becomes clear that in the probability density the azimuthally uniform contribution to the cross section, $F_{\mathrm{UU}, \mathrm{T}}^{h}+\epsilon F_{\mathrm{UU}, \mathrm{L}}^{h}$, has been factored out, which corresponds to normalizing all the Fourier amplitudes to $F_{\mathrm{UU}, \mathrm{T}}^{h}+$ $\epsilon F_{\mathrm{UU}, \mathrm{L}}^{h}$. Setting $F_{\mathrm{UU}, \mathrm{L}}^{h}$ equal to zero, as valid up to subleading twist and leading order in $\alpha_{S}$, results in Fourier amplitudes normalized to

$$
F_{\mathrm{UU}, \mathrm{T}}^{h}=\mathcal{C}\left[f_{1}^{q} D_{1}^{q \rightarrow h}\right]
$$

e.g., to SFA amplitudes of the form $F_{\mathrm{UT}}^{\sin \left(\phi-\phi_{S}\right), h} / F_{\mathrm{UU}, \mathrm{T}}^{h}$. 


\subsubsection{Systematic uncertainties}

Systematic uncertainties in the asymmetry amplitudes arise from

(i) the accuracy of the beam and target polarization measurements,

(ii) the choice of the probability-density function,

(iii) acceptance effects caused by limitations in the geometric acceptance and kinematic requirements in the event selection,

(iv) higher-order QED processes and kinematic smearing effects due to finite spectrometer resolution,

(v) the hadron identification using the RICH detector,

(vi) the stability of the detector over the course of data taking.

In addition, further sources of systematic effects are studied for neutral pions:

(i) variation of the parameterization for the background shape of the two-photon invariant-mass spectrum: third-order Chebyshev polynomial versus Weibull distribution [104],

(ii) variation of the sideband positions with respect to the signal range,

(iii) variation of both sidebands and signal ranges.

The accuracy of the polarization measurements is taken into account as a scale uncertainty on the amplitudes extracted. They amount to $7.3 \%$ and $8.0 \%$ for the single- and double-spin asymmetries, respectively. As they affect both the central values and all other uncertainties as a multiplicative factor, they are provided separately.

Inclusion of additional cosine modulations related to the polarization-averaged cross section, e.g., arising from the Boer-Mulders or Cahn [105] effects, has negligible effects on the single- and double-spin Fourier amplitudes extracted. For that study, an empirical model of those cosine modulations, fit to HERMES data [32], was added to the probability density functions. Furthermore, the results for the single-spin asymmetries extracted using either the full probability density function, e.g., eq. (3.2), or one containing only the single-spin asymmetry terms (as done, e.g., in the previous publications $[29,40]$ ) are fully consistent.

Systematic uncertainties due to experimental acceptance, kinematic smearing, and the hadron identification are estimated simultaneously. Results presented here involve integration over finite bin sizes and partially larger ranges in kinematic variables not explicitly binned in, e.g., in the one-dimensional projections. They are cross-section asymmetries folded with the experimental acceptance, which in general depends on the same set of kinematic variables. They thus represent averages of not only the kinematic dependences of the underlying physics modulations but also include often unaccounted instrumental effects [106]. In particular, these average asymmetries, in general, do not coincide with the 
cross-section asymmetries at the average kinematics provided with each data point. The size of such deviations is estimated using a full Monte Carlo simulation of the experiment based on a version of PyThia6.2 [96, 97] tuned for HERMES kinematics and extended with RADGEN [107] to account for QED radiative effects. The simulation also uses a GEANT3 $[108,109]$ description of the HERMES apparatus, including the beam trajectory and particle deflection in the holding field of the target magnet as well as the efficiency and the cross contamination of the hadron identification using the RICH detector.

The Pythia6.2 event generator does not simulate polarization effects such as those studied here. For this reason, empirical parameterizations (based on a Taylor expansion) of the single- and double-spin asymmetries as a function of $x, Q^{2}, z$, and $P_{h \perp}$ are used to assign a beam-helicity and target-spin state to each semi-inclusive DIS event of the simulation, as detailed in appendix C, to effectively "polarize" the Pyтhia6.2 simulation. The set of parametric models is obtained from fits to the experimental data, separately for each hadron type, using the method described above (section 3.3.1) but now unbinned in all kinematic variables. These models, representing the four-dimensional kinematic dependence $\left(x, Q^{2}, z, P_{h \perp}\right)$ of the ten asymmetry amplitudes of interest, are virtually unaffected by acceptance and instrumental effects, though somewhat biased by the finite number of terms included in the fit (compared to the a priori infinite Taylor expansion).

The systematic uncertainties for the combined effect of limited acceptance, higher-order QED effects, and the hadron identification using the RICH detector are then estimated from the difference of the asymmetry amplitudes extracted from the simulated data and their model evaluated at their average kinematic values (further details are provided in appendix C). These systematic uncertainties thus correspond to the case of interpreting the data as asymmetry values for the given average kinematics in each bin, in contrast to ratio of cross sections integrated over all the kinematics in the ranges applicable to each particular bin.

The stability of the results was tested in various ways: comparing the results extracted for positron and electron beam separately, and comparing double-spin asymmetries for periods of different beam-helicity states. The studies found in general full consistency of the data for different beam charges as well as of the data for different beam helicities. The only notable exception are the $\pi^{0}$ results. Various statistical tests result in a statistical incompatibility at $90 \%$ confidence level $^{14}$ for the one-dimensional extraction of the Sivers case, with hints of statistical incompatibility for some of the other modulations. As a consequence, conservatively, half the difference between constant fits to the $\pi^{0}$ results from electron and positron data are assigned as additional systematic uncertainties. They are added in quadrature to those related to other instrumental effects and kinematic smearing.

The remaining sources of systematic uncertainties considered are found to have a negligible effect on the results.

\footnotetext{
${ }^{14}$ The results of these tests are, however, limited by the small number of data points.
} 


\subsection{Differences with previous analyses}

Though the general framework has much in common with that in the prior HERMES publications on the Sivers and Collins effects [29, 40], there are several obvious differences and some minor improvements in the data analysis:

(i) The analysis is based on a later data production, which among others included updated tracking and alignment information, as well as corrections for minimal beamenergy variations.

(ii) The first such measurement of asymmetries for protons and antiprotons is presented.

(iii) The extraction of asymmetries for neutral pions is improved in various aspects, among others a different treatment of photons that start showering already in the pre-shower detector and adjusted ranges in the two-photon invariant mass used for the signal and the background subtraction. Also, only photon pairs that are detected in the same detector half are kept in the analysis.

(iv) The analysis is performed in a three-dimensional kinematic binning; the $x$ range is extended to an upper limit of 0.6 .

(v) The one-dimensional binning has been adapted to permit extraction of asymmetry amplitudes for also the low-statistic hadrons; in addition, the binning in $z$ is extended to include the high- $z$ region of $0.7<z<1.2$.

(vi) The extraction of all the various SSA and DSA is performed in one combined fit to minimize potential cross talk between moments.

(vii) The standard set of results comprises the structure-function asymmetries and thus includes corrections for the $\epsilon$-dependent kinematic prefactors.

\section{Results and interpretation}

The SSA and DSA amplitudes are extracted in a three-dimensional kinematic binning in $x, z$, and $P_{h \perp}$ to allow the exploration of correlated dependences. In comparison to earlier measurements, e.g., in refs. [29, 40], this provides measurements in kinematic corners that are suppressed when integrating over all but one variable. Three further principal advancements are worth mentioning: (i) the total number of data points per particle species increases to 64 , (ii) none of those 64 data points is statistically correlated with any of the other, ${ }^{15}$ and (iii) the multi-dimensional binning avoids integration over large regions of the kinematic space and results in a much reduced systematic uncertainty. In particular the latter two should significantly increase the reliability of uncertainties resulting from phenomenological fits to combined data of one-dimensional projections as the latter have an unspecified degree of statistical and systematic correlation.

\footnotetext{
${ }^{15}$ While data points for one particular azimuthal moment are uncorrelated, results for the different azimuthal moments in one kinematic bin may still be correlated. That degree of correlation is provided in the supplementary material.
} 


\begin{tabular}{|cl|ccccc|cc|}
\hline \multicolumn{2}{|c|}{ Azimuthal modulation } & \multicolumn{8}{|c|}{ Significant non-vanishing Fourier amplitude } \\
& & $\pi^{+}$ & $\pi^{-}$ & $K^{+}$ & $K^{-}$ & $p$ & $\pi^{0}$ & $\bar{p}$ \\
\hline $\sin \left(\phi+\phi_{S}\right)$ & {$[$ Collins] } & $\checkmark$ & $\checkmark$ & $\checkmark$ & & $\checkmark$ & & \\
$\sin \left(\phi-\phi_{S}\right)$ & {$[$ Sivers] } & $\checkmark$ & & $\checkmark$ & $\checkmark$ & $\checkmark$ & $(\checkmark)$ & $\checkmark$ \\
$\sin \left(3 \phi-\phi_{S}\right)$ & {$[$ Pretzelosity] } & & & & & & & \\
$\sin \left(\phi_{S}\right)$ & & $(\checkmark)$ & $\checkmark$ & & $\checkmark$ & & & \\
$\sin \left(2 \phi-\phi_{S}\right)$ & & & & & & & & $(\checkmark)$ \\
$\sin \left(2 \phi+\phi_{S}\right)$ & & & & $\checkmark$ & & & & \\
$\cos \left(\phi-\phi_{S}\right)$ & [Worm-gear] & $\checkmark$ & $(\checkmark)$ & $(\checkmark)$ & & & & \\
$\cos \left(\phi+\phi_{S}\right)$ & & & & & & & & \\
$\cos \left(\phi_{S}\right)$ & & & & $\checkmark$ & & & & \\
$\cos \left(2 \phi-\phi_{S}\right)$ & & & & & & & & \\
\hline
\end{tabular}

Table 9. The various azimuthal modulations of the semi-inclusive cross section and those hadron species whose corresponding Fourier amplitudes are incompatible with the NULL hypothesis at 95\% (90\%) confidence. Antiprotons and $\pi^{0}$ are given separated in the last two columns to indicate that the statistical test of those is based on the one-dimensional projections and hence restricted to using only seven data points.

Due to the more limited precision of the antiproton and neutral-pion data, such threedimensional kinematic binning was not feasible. They were thus analyzed as functions of $x$, $z$, and $P_{h \perp}$ individually (cf. tables 7 and 8), integrating over the corresponding remaining kinematic variables.

Asymmetries in one overall kinematic bin are not presented as their extraction suffers from the largest acceptance effects. They are also of limited value for phenomenology. Instead, the results for all asymmetries were tested against the NULL hypothesis using the two-sided Student's t-test. The asymmetry results binned in three dimensions were used, where available, to increase the robustness of the Student's t-test by using 64 data points and avoiding cancelation effects from integrating over kinematic dependences. In the case of $\pi^{0}$ and antiprotons, where results in only the one-dimensional binning are available, they are considered to be inconsistent with zero if the Student's t-test established this for at least one of the three projections (versus $x, z$, or $P_{h \perp}$ ). ${ }^{16}$ It is found that most asymmetry amplitudes are consistent with zero in the semi-inclusive region $0.2<z<0.7$ used here. Those asymmetry amplitudes that are found to be inconsistent with zero at 95\% (90\%) confidence level are listed in table 9. Significantly non-zero results were neither found for the pretzelosity $2\left\langle\sin \left(3 \phi-\phi_{S}\right)\right\rangle_{\mathrm{U} \perp}^{h}$ Fourier amplitudes nor for the $M / Q$-suppressed $2\left\langle\cos \left(\phi+\phi_{S}\right)\right\rangle_{\mathrm{L} \perp}^{h}$ and $2\left\langle\cos \left(2 \phi-\phi_{S}\right)\right\rangle_{\mathrm{L} \perp}^{h}$ Fourier amplitudes. For the $2\left\langle\sin \left(2 \phi-\phi_{S}\right)\right\rangle_{\mathrm{U} \perp}^{h}$ Fourier amplitude, only antiprotons were found to be inconsistent with the NULL hypothesis and this only at the $90 \%$ but not at the $95 \%$ confidence level.

\footnotetext{
${ }^{16}$ It has to be kept in mind that the Student's t-test becomes less reliable when using a small number of data points as, e.g., the case for the one-dimensional binning.
} 


\begin{tabular}{|lccccc|}
\hline Hadron & \multicolumn{5}{c}{ Mean values of kinematic variables } \\
& $\left\langle Q^{2}\right\rangle$ & $\langle x\rangle$ & $\langle y\rangle$ & $\langle z\rangle$ & $\left\langle P_{h \perp}\right\rangle$ \\
\hline$\pi^{+}$ & $2.445 \mathrm{GeV}^{2}$ & 0.095 & 0.544 & 0.362 & $0.394 \mathrm{GeV}$ \\
$\pi^{0}$ & $2.506 \mathrm{GeV}^{2}$ & 0.089 & 0.588 & 0.357 & $0.396 \mathrm{GeV}$ \\
$\pi^{-}$ & $2.366 \mathrm{GeV}^{2}$ & 0.092 & 0.548 & 0.354 & $0.393 \mathrm{GeV}$ \\
$K^{+}$ & $2.524 \mathrm{GeV}^{2}$ & 0.097 & 0.548 & 0.391 & $0.417 \mathrm{GeV}$ \\
$K^{-}$ & $2.381 \mathrm{GeV}^{2}$ & 0.089 & 0.569 & 0.356 & $0.412 \mathrm{GeV}$ \\
$p$ & $2.595 \mathrm{GeV}^{2}$ & 0.095 & 0.574 & 0.421 & $0.452 \mathrm{GeV}$ \\
$\bar{p}$ & $2.393 \mathrm{GeV}^{2}$ & 0.076 & 0.655 & 0.364 & $0.477 \mathrm{GeV}$ \\
\hline
\end{tabular}

Table 10. Mean kinematic values for pions, charged kaons, as well as for protons and antiprotons in the standard semi-inclusive range $0.2<z<0.7$.

In the following, the most important observations and features of the data are discussed. ${ }^{17}$ The corresponding mean kinematics for the kinematic region covered within the standard semi-inclusive selection are listed in table 10 .

The error bars in the following figures indicate the statistical uncertainties of the SSA and DSA Fourier amplitudes. The uncertainty bands represent the systematic uncertainties of the results arising from acceptance, finite detector resolution, higher-order QED effects, possible misidentification of hadrons, and detector instabilities (the latter only for $\pi^{0}$, while negligible for all other hadrons). In addition, the uncertainties arising from the measurement precision of beam and target polarization are provided separately as an overall scale uncertainty: $7.3 \%$ in the case of SSA amplitudes and $8.0 \%$ for the DSA amplitudes.

\subsection{Signals for transversity and the Collins fragmentation function}

Non-vanishing $\sin \left(\phi+\phi_{S}\right)$ modulations ("Collins asymmetries") are evidence for two chiralodd TMDs: the transversity distribution and the naive- $T$-odd Collins fragmentation function. Both have been subject to intense experimental and theoretical studies, also at HERMES, which first reported evidence for those [28]. Results for pions, charged kaons, and the pion charge-difference CSA were reported in ref. [29] for one-dimensional projections in $x, z$, and $P_{h \perp}$. The most striking feature of those results is a large negative asymmetry for negative pions, opposite in sign and even larger in magnitude in comparison to the asymmetry for positive pions. These results were explained [28] by a large disfavored Collins function, describing, e.g., the fragmentation of up quarks into negative pions, that is opposite in sign to the favored Collins function. This explanation was later confirmed by phenomenological fits [110-116] to various data sets on semi-inclusive deep-inelastic scattering [28, 29, 41, 117-122] and on $e^{+} e^{-}$annihilation into hadrons [123-126], as well as on hadron collisions in the case of ref. [116]. While earlier work employed simplified approaches for the Dokshitzer-Gribov-Lipatov-Altarelli-Parisi evolution in the fits to data at various scales, the focus has moved to employ TMD evolution in more recent works, especially in view of the $B$-factory data at $Q^{2} \sim 100 \mathrm{GeV}^{2}$.

\footnotetext{
${ }^{17}$ The complete set of results are provided as supplementary material, figures of those can be obtained from HERMES management.
} 

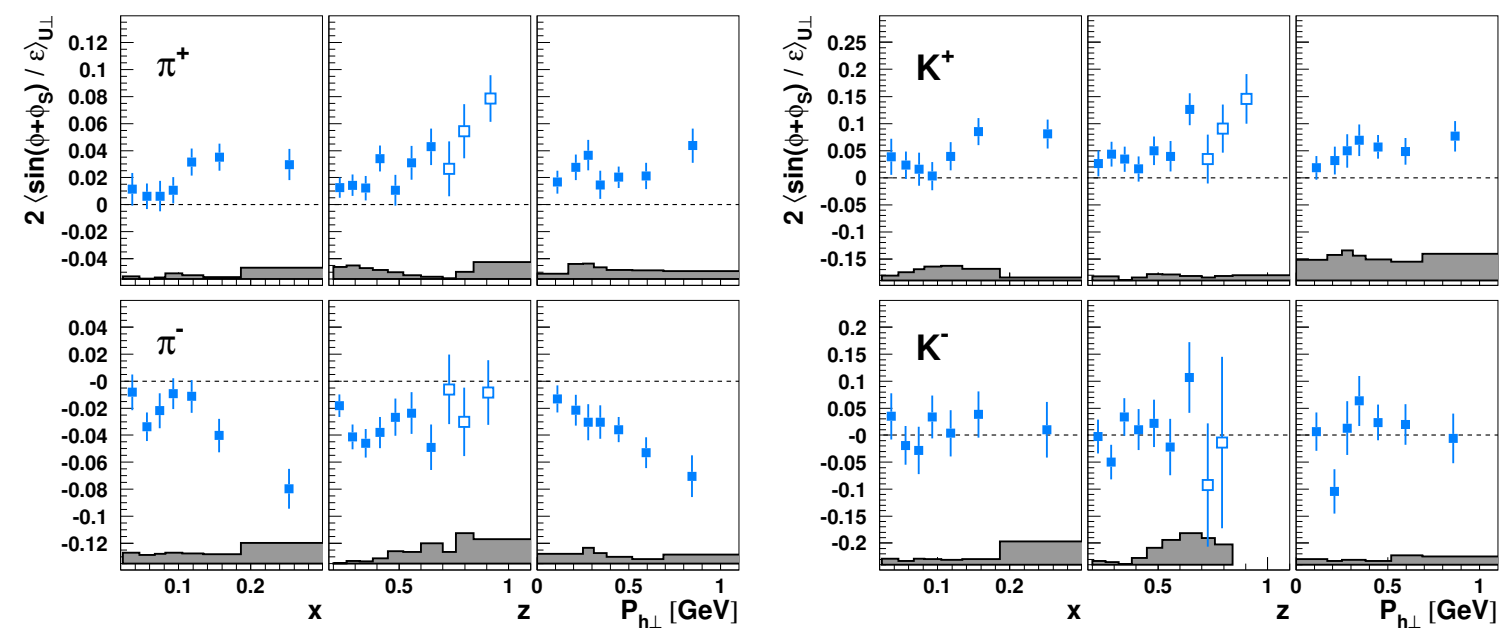

Figure 8. Collins SFA for charged mesons (left: pions; right: kaons) presented either in bins of $x$, $z$, or $P_{h \perp}$. Data at large values of $z$, marked by open points in the $z$ projection, are not included in the other projections. Systematic uncertainties are given as bands, not including the additional scale uncertainty of $7.3 \%$ due to the precision of the target-polarization determination.

The results for the transversity distributions from global fits are of the same $\operatorname{sign}^{18}$ as results for the helicity distribution, but somewhat smaller in magnitude, by as much as a factor of two for the $d$-quark distribution. Flavor decompositions of the collinear transversity distribution, based on analysis of dihadron production in semi-inclusive deep-inelastic scattering [127-129], $e^{+} e^{-}$annihilation [130], and more recently in $p^{\uparrow} p$ collision [131], confirm this general behavior [132-135]. In general, the $d$-quark transversity distribution is much less constrained, given the $u$-quark dominance in many of the processes employed in the extractions. It is interesting to remark that all phenomenological extractions of the transversity distribution present some discrepancies with respect to lattice predictions, especially for what concerns the $u$-quark contribution to the nucleon tensor charge (see, e.g., refs. [136-138]).

The Collins asymmetries extracted here for mesons in one-dimensional projections resemble to a high degree those published previously [29]. This is expected as based on the same data set, though involving a number of analysis improvements (cf. section 3.4). The most significant advancement in the measurement of the SFA shown in figure 8 is the inclusion of the $\epsilon$-dependent kinematic prefactors in the probability density function (3.3) of the maximum-likelihood fit. This leads on average to an amplification of the asymmetry magnitude as, in the case of the Collins asymmetry, this prefactor is smaller than unity and thus diminishes the transversity/Collins-induced modulation.

The Collins asymmetries for charged pions are opposite in sign and increasing with $x$, which can be attributed to transversity predominantly being a valence-quark effect. The dependence on $z$ in the semi-inclusive range is a clear increase with $z$ for $\pi^{+}$, while first clearly increasing but then leveling out for $\pi^{-}$. As expected, the asymmetries increase

\footnotetext{
${ }^{18}$ Note that the absolute sign can not be determined unambiguously due to the chiral-odd nature of both transversity and the Collins fragmentation function.
} 
with $P_{h \perp}$ at low values of $P_{h \perp}$. This rise continues in the case of $\pi^{-}$up to the highest $P_{h \perp}$ values probed here. In contrast, for $\pi^{+}$there is a hint of a plateau after the initial rise with $P_{h \perp}$.

In the case of strange mesons, positive kaons exhibit larger though in shape similar Collins asymmetries when compared to those for positive pions. In contrast, the Collins asymmetries for negative kaons are found to be consistent with zero. Assuming that the nucleon's sea-quark transversity distributions are vanishing (or small), only disfavored fragmentation of up and down quarks can contribute to the $K^{-}$moments. Being disfavored fragmentation, the contribution is expected to be suppressed. Furthermore, being of opposite sign, the up and down contributions cancel to a large extend. Recently, data from $e^{+} e^{-}$annihilation into kaons and pions [139] were analyzed and the Collins fragmentations functions extracted were then used for the estimate of the Collins asymmetries in semi-inclusive deep-inelastic scattering. Indeed, a largely vanishing $K^{-}$ Collins asymmetry, as observed here, was found considering only valence transversity as non-vanishing [140]. The data for kaons are interesting in the context of chiral symmetry breaking in QCD, where pions and kaons are considered to be the Goldstone bosons. In the chiral limit, fragmentation into pions and kaons should be the same, in particular, $H_{1}^{\perp, q \rightarrow \pi}(z)=H_{1}^{\perp, q \rightarrow K}(z)$ [141]. In reality, this is already violated in the case of unpolarized fragmentation, e.g., $D_{1}^{u \rightarrow \pi^{+}}(z)>D_{1}^{u \rightarrow K^{+}}(z)$. Extractions of the Collins fragmentation function for both pions and kaons will shed light on the (better) validity of the chiral limit for the case of the Collins fragmentation function.

The one-dimensional dependences of the Collins asymmetries measured by the COMPASS Collaboration [121] are consistent with the ones reported here, apart from the $K^{-}$ asymmetries, which are non-vanishing and negative ${ }^{19}$ at COMPASS. The kaon Collins asymmetries from Jefferson Lab for transversely polarized ${ }^{3} \mathrm{He}$, effectively a target of transversely polarized neutrons, are consistent with zero within large uncertainties, with a hint of a sizable negative asymmetry for $K^{-}$[142].

Two examples for the three-dimensionally binned data are presented in figures 9 and 10 . The $\pi^{-}$Collins asymmetries are plotted either versus $x$ (figure 9) or versus $z$ (figure 10), revealing a merely weak dependence on $z$ but an $x$ dependence that is pronounced, mainly at large $P_{h \perp}($ and $z)$.

As discussed above, the Collins fragmentation functions extracted in phenomenological fits are opposite in sign and similar in magnitude for $\pi^{+}$and $\pi^{-}$. The $\pi^{0}$ Collins fragmentation function can be related through isospin symmetry to the ones of charged pions. In particular, it is the average of the latter two and thus approximately vanishes. The $\pi^{0}$ Collins asymmetries, available only as one-dimensional projections, are shown in figure 11. They are indeed consistent with zero as expected.

The proton and antiproton Collins asymmetries, measured here for the first time, are depicted in figure 11 as one-dimensional projections. They are mostly negative in case of protons, while the antiproton results are consistent with zero. The Collins effect is a fragmentation effect, it might be suppressed for spin- $\frac{1}{2}$ hadron production as compared to

\footnotetext{
${ }^{19}$ Note that COMPASS uses a different sign convention for the transversity-induced asymmetries.
} 


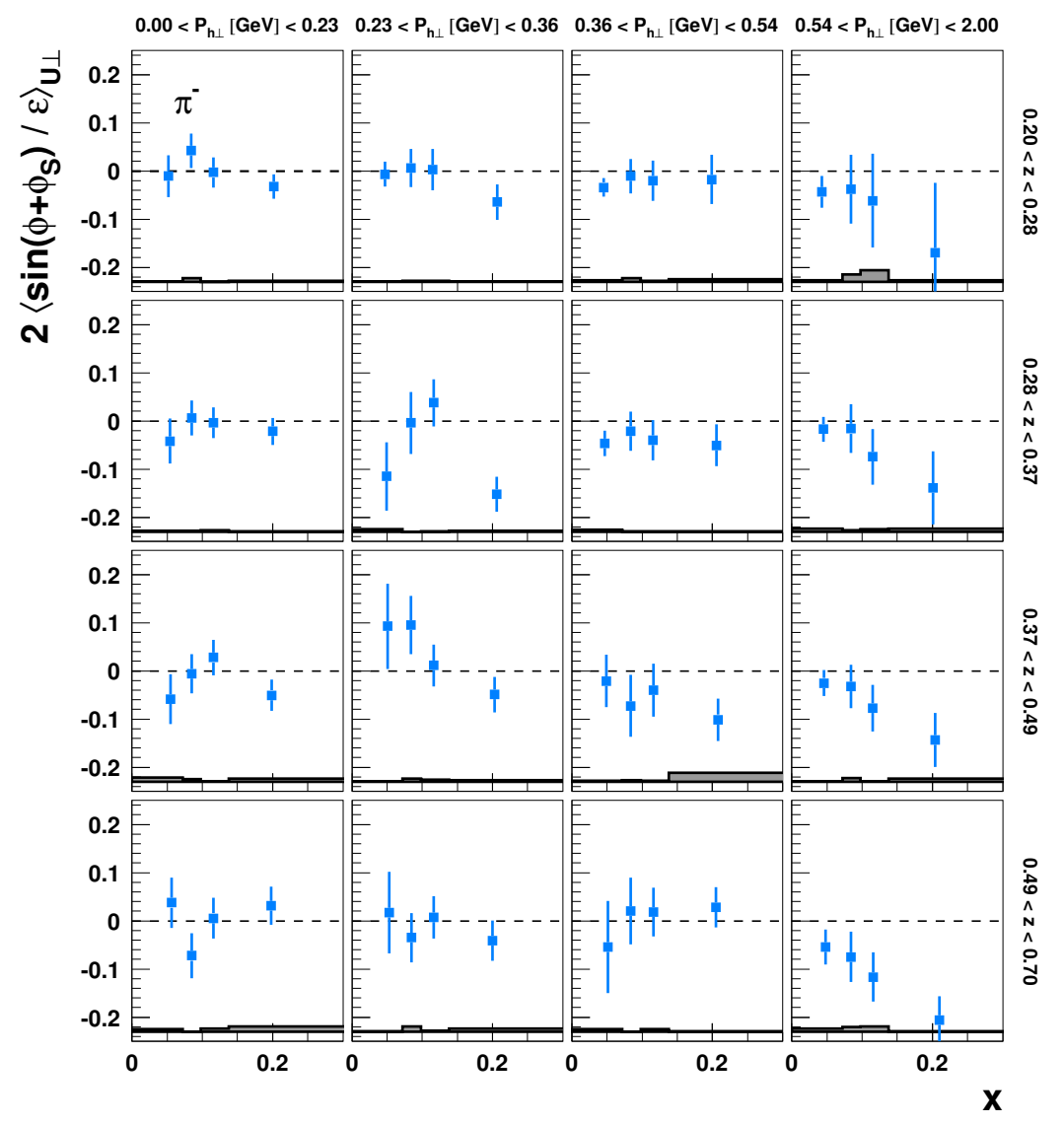

Figure 9. Collins SFA for $\pi^{-}$extracted simultaneously in bins of $x, z$, and $P_{h \perp}$, presented as a function of $x$. Systematic uncertainties are given as bands, not including the additional scale uncertainty of $7.3 \%$ due to the precision of the target-polarization determination.

meson production. Models provide little guidance, and were already severely challenged by the large disfavored Collins fragmentation function for pions. In the Artru approach [143], the transverse momentum of pions arises through an interplay of the meson and quark spins, as well as the vacuum structure: $q \bar{q}$ pairs produced in the string-fragmentation model [144] are produced with vacuum quantum numbers, i.e., their spins are aligned and possess one unit of angular momentum opposite to their spin orientation. This orbital angular momentum is partially transformed into transverse momentum of the produced pion when pairing one of those quarks with the fragmenting quark, with the quark spins anti-aligned to form a spin-zero pion. If a favored pion forms in the first string break, a disfavored pion from the next break will inherit transverse momentum from the first break in the direction opposite to that acquired by the first pion, leading to a disfavored Collins function that is opposite in sign to that of the favored Collins function, consistent with the data. The Collins function for baryons, however, is more difficult to predict in this approach as, e.g., the role of diquark production in the fragmentation process or diquark fragmentation is far less understood. The more complex production might thus easily wash out any transverse-polarization dependence of the fragmentation process. More recently, 


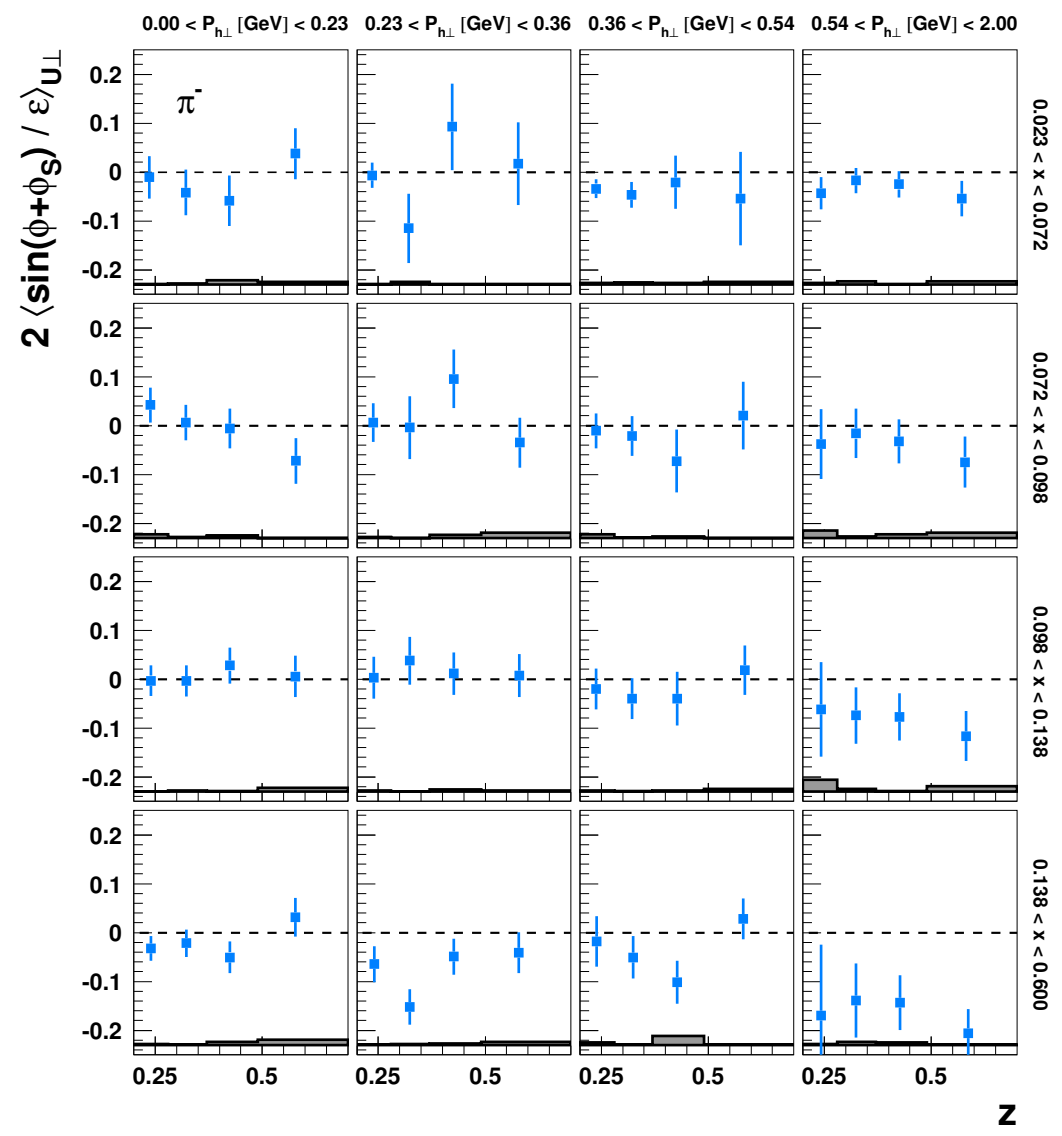

Figure 10. Collins SFA for $\pi^{-}$extracted simultaneously in bins of $x, z$, and $P_{h \perp}$, presented as a function of $z$. Systematic uncertainties are given as bands, not including the additional scale uncertainty of $7.3 \%$ due to the precision of the target-polarization determination.
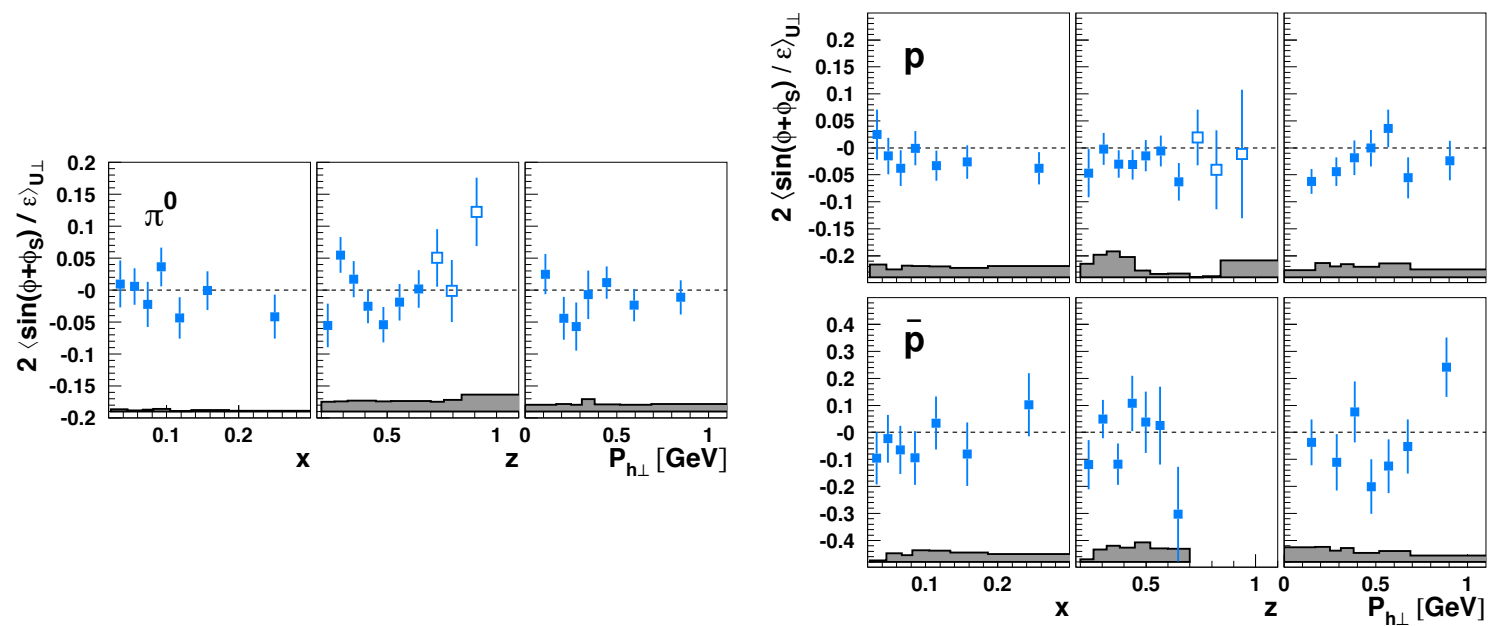

Figure 11. Collins SFA for $\pi^{0}$ (left), protons, and antiprotons (right) presented either in bins of $x$, $z$, or $P_{h \perp}$. Data at large values of $z$, marked by open points in the $z$ projection, are not included in the other projections (no such high- $z$ points are available for antiprotons due to a lack of precision). Systematic uncertainties are given as bands, not including the additional scale uncertainty of $7.3 \%$ due to the precision of the target-polarization determination. 
a calculation in a diquark spectator model resulted in sizable Collins functions for up and down quarks into $\Lambda$ hyperons [145]. While no such calculation is presented for the case of protons, it is not unplausible that it would result in a non-vanishing Collins effect, as hinted at by the data.

Lastly, looking at the "semi-exclusive" large- $z$ region (figures 8 and 11), the asymmetries for positive mesons follow the trend of increasing with $z$ all the way to the highest $z$, while such behavior is not visible for the other hadrons. ${ }^{20}$ With increasing $z$ disfavored fragmentation decreases in importance. As a result the sensitivity to the struck quark - mainly up quarks - increases, leading to a further enhancement of the $\pi^{+}$and $K^{+}$ asymmetries.

\subsection{Evidence for the Sivers function}

The naive-T-odd Sivers effect, first observed in semi-inclusive deep-inelastic scattering by HERMES for positive pions [28], has been discussed already in detail in ref. [40], where one-dimensional projections versus $x, z$, and $P_{h \perp}$ of the $\sin \left(\phi-\phi_{S}\right)$ Fourier amplitudes were presented for pions, charged kaons, and the pion charge difference. Significantly positive asymmetries were observed for positive pions and kaons, again larger for kaons than for pions. Significant positive values were also seen for $\pi^{0}$ as well as the pion charge-difference asymmetry, while results for negative pions and kaons were found to be consistent with zero. These findings were interpreted as originating from up and down valence-quark Sivers distributions that are opposite in sign, in accordance with the prediction [45] based on the quark contributions to the proton's anomalous magnetic moment. Phenomenological fits $[46,116,146-157]$ to the HERMES and other semi-inclusive deep-inelastic scattering data $[41,56,118,120-122,158]$ (as well as to hadron-collision data in the case of ref. [116]) mainly result in Sivers distributions that are indeed significant only for valence quarks. ${ }^{21}$ Those fit results suggest that valence quarks are sufficient to saturate the Burkardt sum rule $[159,160]$, which states that the net transverse momentum carried by partons inside a transversely polarized nucleon (which is related to the Sivers function) vanishes when summing over all partons (quarks and gluons).

The Sivers asymmetries extracted here for charged pions and kaons in one-dimensional projections are presented in figure 12. The Sivers modulation is the only one analyzed here that does not involve an $\epsilon$-dependent kinematic prefactor, i.e., SFA and CSA should coincide. This is indeed found up to negligible variations introduced through correlations with other modulations in the fit. Hence, even though the previously published results [40] were obtained from a fit of the CSA to the data, while the ones shown in this section are the outcome of the SFA fit, the slight differences between them - though consistent stem solely from the updated analysis (changes in binning, newer calibrations of the data, etc.).

\footnotetext{
${ }^{20}$ Due to insufficient yields, results for only two high- $z$ bins are available for $K^{-}$and none for antiprotons.

${ }^{21}$ In ref. [157] only the $u$-quark Sivers function is unambiguously found non-zero and the experimental data can be described with assigning the still required contributions either to $d$ quarks or to the other remaining parton flavors, with further data needed for a more conclusive evaluation of the situation.
} 

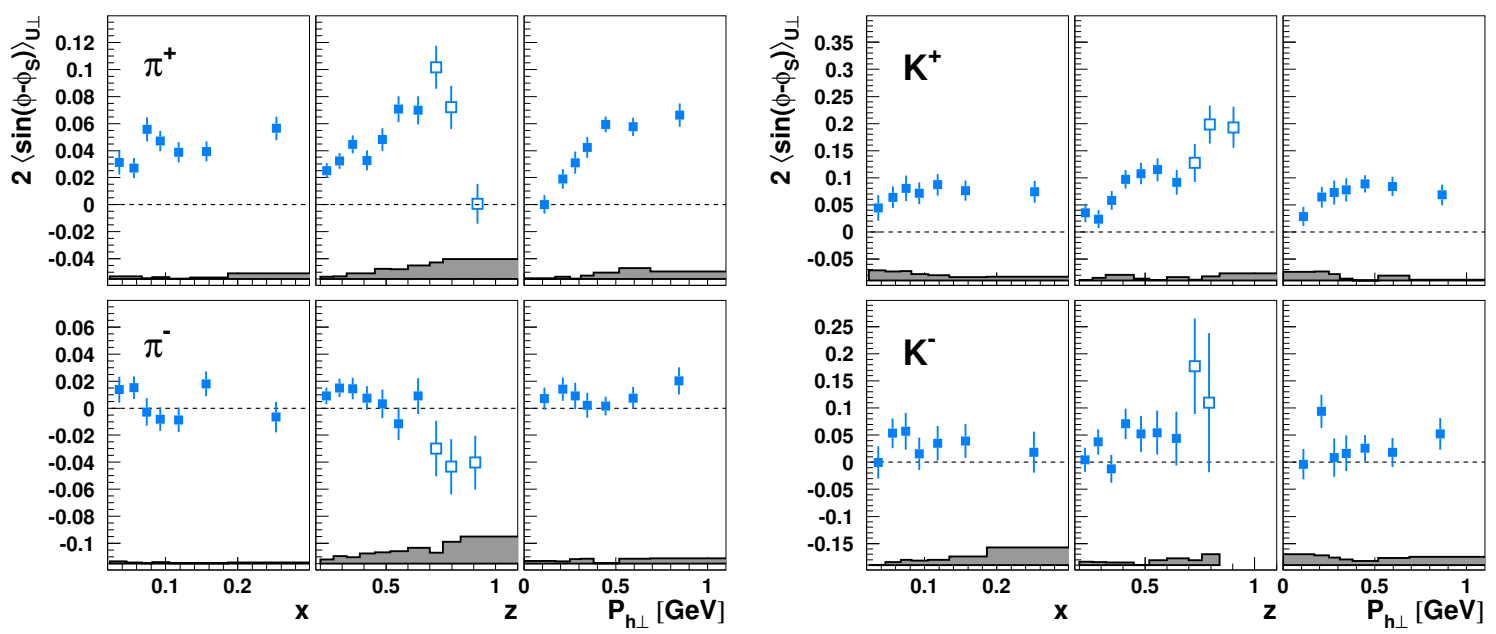

Figure 12. Sivers SFA for charged mesons (left: pions; right: kaons) presented either in bins of $x$, $z$, or $P_{h \perp}$. Data at large values of $z$, marked by open points in the $z$ projection, are not included in the other projections. Systematic uncertainties are given as bands, not including the additional scale uncertainty of $7.3 \%$ due to the precision of the target-polarization determination.

As in the previous publication [40], significantly positive Sivers amplitudes are observed for positive pions. The asymmetries rise slightly with $x$, though remain significantly nonzero even at the lowest $x$ values probed in this experiment. The rise with $z$ and $P_{h \perp}$ is much more pronounced. However, while the rise continues throughout the semi-inclusive $z$ range, it is leveling off at larger values of $P_{h \perp}$.

The $\pi^{-}$Sivers asymmetry in the one-dimensional $x$ projection is consistent with zero. While $\pi^{+}$electroproduction off protons is dominated by up-quark scattering, $\pi^{-}$receives large contributions from down quarks. The vanishing Sivers asymmetry for negative pions can thus be understood as a cancelation of a Sivers effect that is opposite in sign for up and down quarks. This may also explain the peculiar behavior of the $z$ dependence: at low values of $z$ disfavored fragmentation plays a significant role and thus contributions from up quarks can push the asymmetry towards positive values. At large values of $z$, however, disfavored fragmentation dies out and the favored production off down quarks prevails leading to a negative asymmetry. Some caution with this argumentation is deserved as at large values of $z$, the contribution from the decay of exclusive $\rho^{0}$ electroproduction to both the $\pi^{+}$and $\pi^{-}$samples becomes sizable, as can be concluded from a Pyтнia6.2 Monte Carlo simulation (cf. figure 4), even more so for $\pi^{-}$than for $\pi^{+}$. Charge-conjugation dictates that the decay pions from the $\rho^{0}$ exhibit the same asymmetry regardless of their charge. ${ }^{22}$ Examining the large- $z$ behavior of the charged-pion asymmetries, indeed a clear change of trend can be observed for positive pions. Still, the significant difference between the charged-pion asymmetries over most of the kinematic range suggests that the nonvanishing asymmetries observed are not driven merely by exclusive $\rho^{0}$ electroproduction.

The $K^{+}$Sivers asymmetry follows a similar kinematic behavior as the one for $\pi^{+}$, but is larger in magnitude, as can be seen in figure 13. While $u$-quark scattering should domi-

\footnotetext{
${ }^{22}$ This is also one motivation for looking at the charge-difference asymmetry in ref. [40] in which such contributions cancel.
} 


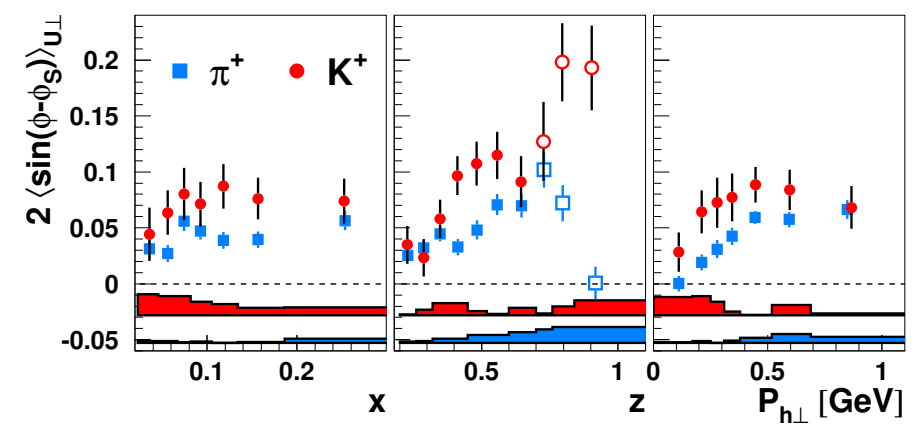

Figure 13. Comparison of Sivers SFA for positive pions (squares) and kaons (circles) presented either in bins of $x, z$, or $P_{h \perp}$. Data at large values of $z$, marked by open points in the $z$ projection, are not included in the other projections. Systematic uncertainties are given as bands, not including the additional scale uncertainty of $7.3 \%$ due to the precision of the target-polarization determination.

nate production off protons of both positive pions and kaons, various differences between pion and kaon production might point to the origin for the larger $K^{+}$asymmetry: (i) differences in the relative strengths of the disfavored $d$-quark fragmentation compared to the favored $u$-quark fragmentation for positive pions and kaons might lead to a reduced canceling contribution from the $d$-quark Sivers function; (ii) in general, differences in the role of sea quarks; (iii) differences - as hinted in a phenomenological analysis [161] of HERMES multiplicity data [98] — in the transverse-momentum dependence of hadronization for different quark flavors that enters the convolution over transverse momentum in eq. (2.6); (iv) and also higher-twist effects as it was observed in ref. [40] that the $\pi^{+}-K^{+}$difference was more pronounced at lower values of $Q^{2}$. Notwithstanding those differences, acknowledging $u$-quark dominance in both $\pi^{+}$and $K^{+}$production and relating their positive Sivers asymmetries to eq. (2.6) leads immediately to the conclusion that the $u$-quark Sivers function, $f_{1 \mathrm{~T}}^{\perp, u}$, must be negative. Adding the $\pi^{-}$data, as argued before, results in a positive $f_{1 \mathrm{~T}}^{\perp, d}$.

Looking at the newly explored large- $z$ region, the similarity of $\pi^{+}$and $K^{+}$Sivers asymmetries disappears: in contrast to the drop at large $z$ of the asymmetry values in the case of positive pions, the $K^{+}$Sivers asymmetry continues its trend to increase with $z$, which is indeed the expected behavior. This divergence of behavior for positive pions and kaons can also be seen in the corresponding data of the COMPASS Collaboration [121], in particular in the $x$ region overlapping with HERMES. As decay products from exclusively produced vector-mesons contribute significantly less to $K^{+}$production, this might be another indication of a non-negligible role of those in the case of the pion data.

While the data on negative kaons is more limited in precision, also here a positive asymmetry is clearly visible in the right plot of figure 12. Negative kaons and the target proton have no valence quarks in common. While sensitive to the nucleon's sea-quark, $u$-quark scattering will still be a dominant contribution, as can be concluded from the $K^{-}$ purity in ref. [162]. However, in contrast to $K^{+}$, the $u$-quark contribution is suppressed and diluted ${ }^{23}$ in the case of the $K^{-}$asymmetry.

\footnotetext{
23 "Diluted" in the literal sense or through competing/canceling contributions from other quark flavors, e.g., $d$-quarks.
} 


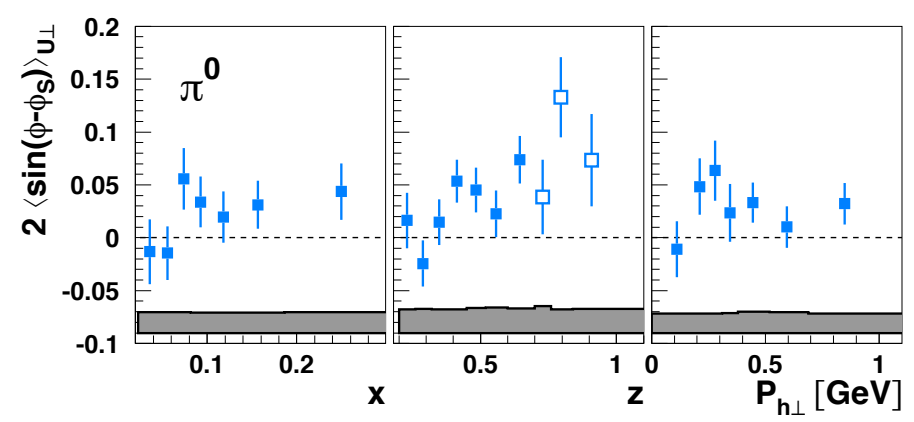

Figure 14. Sivers SFA for $\pi^{0}$ presented either in bins of $x, z$, or $P_{h \perp}$. Data at large values of $z$, marked by open points in the $z$ projection, are not included in the other projections. Systematic uncertainties are given as bands, not including the additional scale uncertainty of $7.3 \%$ due to the precision of the target-polarization determination.

As is the case for $K^{-}$, the $\pi^{0}$ results, presented in figure 14, have poor statistical precision but still indicate a positive asymmetry. This can be expected from the results for charged pions due to isospin symmetry in semi-inclusive deep-inelastic scattering. In the high- $z$ range, the $\pi^{0}$ asymmetries remain positive around $5-10 \%$, thus not following the strongly falling trend of the $\pi^{+}$asymmetries. Also here the contribution from exclusive vector-meson production is much smaller than for $\pi^{+}$(cf. figure 4); thus, an interpretation in terms of ordinary fragmentation is likely much more applicable, leading to a positive asymmetry due to $u$-quark dominance.

Figure 15 shows, as an illustrative example, the Sivers asymmetry for $\pi^{+}$mesons in the three-dimensional binning, compared to a phenomenological fit [152]. The latter, being based on previous versions of these data (as well as data from COMPASS), describes the overall behavior well. The multi-dimensional binning as well as the much reduced systematics of the data presented here should help to better constrain future phenomenological analyses.

In figure 16, the first measurement of Sivers asymmetries for proton and antiprotons is presented. A clearly positive Sivers asymmetry is observed for protons. Also the less precise antiproton data favor a positive Sivers asymmetry. Baryon production is a less understood process at lower center-of-mass energies. Therefore, care must be taken when interpreting those in the usual factorized way. Leaving this warning aside and assuming quark fragmentation as the dominant process here, $u$-quark fragmentation prevails proton production, and - having no valence quark in common with the target proton - antiprotons as well are likely to originate from $u$-quarks, in particular at these values of $x$, where sea quarks are still scarce in the target proton. Dominance of $u$-quarks in proton and antiproton leptoproduction is supported by results from global fits of fragmentation functions [163]. The Sivers effect is sometimes referred to as a "quark-jet effect", e.g., already before forming the final hadron, the transverse-momentum distribution of the fragmenting quark exhibits the Sivers signature of a left-right asymmetry with respect to the direction of the target polarization. It is thus natural to expect similar asymmetries for "current-fragmentation" protons and antiprotons as those for the other hadrons whose electroproduction off the 


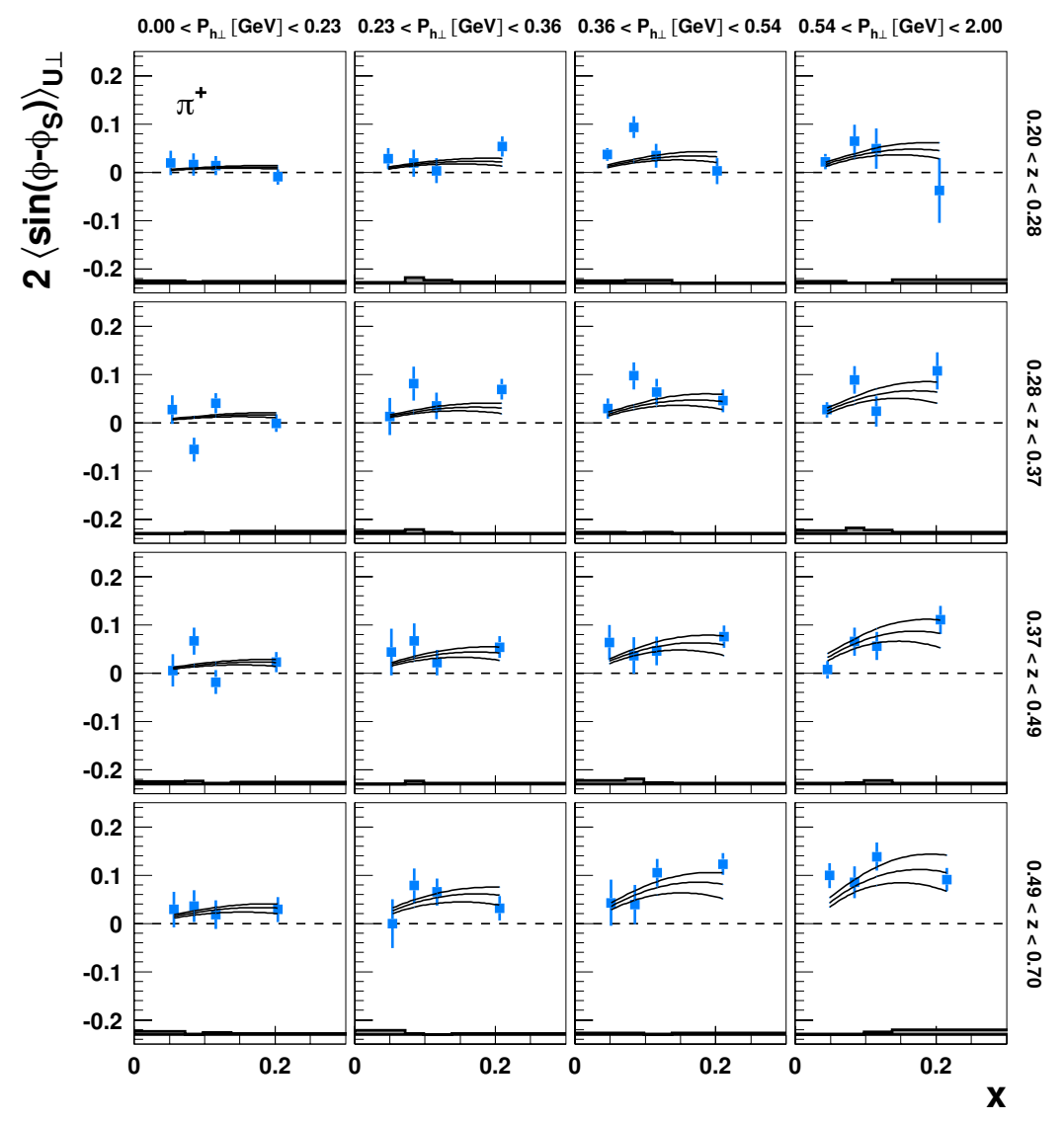

Figure 15. Sivers SFA for $\pi^{+}$extracted simultaneously in bins of $x, z$, and $P_{h \perp}$, presented as a function of $x$. Systematic uncertainties are given as bands, not including the additional scale uncertainty of $7.3 \%$ due to the precision of the target-polarization determination. Overlaid is a phenomenological fit [152] to previously available data, with the three lines corresponding to the central value of the fit and the fit uncertainty.

proton is dominated by $u$-quark scattering [164]. Figure 17 compares the Sivers asymmetries for both protons and antiprotons with those for positive pions. Within the available precision an almost surprising agreement of proton and $\pi^{+}$asymmetries is visible. Also the asymmetries for antiprotons are very similar, however, the present measurement is plagued by large uncertainties.

In order to investigate slightly more the nature of proton and antiproton production at HERMES, figure 18 depicts the ratio of their raw production rates, e.g., yields not corrected for instrumental effects. The sudden increase of the proton-over-antiproton ratio towards very low $z$ might indicate the onset of target fragmentation, while in most of the $z$ range studied here the ratio exhibits a behavior consistent with current fragmentation. In particular, with increasing $z$ the production of antiprotons, which have no valence quarks in common with the target nucleons, is increasingly suppressed compared to protons. A second qualitative argument supporting the hypothesis of dominance of current fragmentation is the sign of the Sivers asymmetry for protons. The current jet is dominated by $u$-quark 

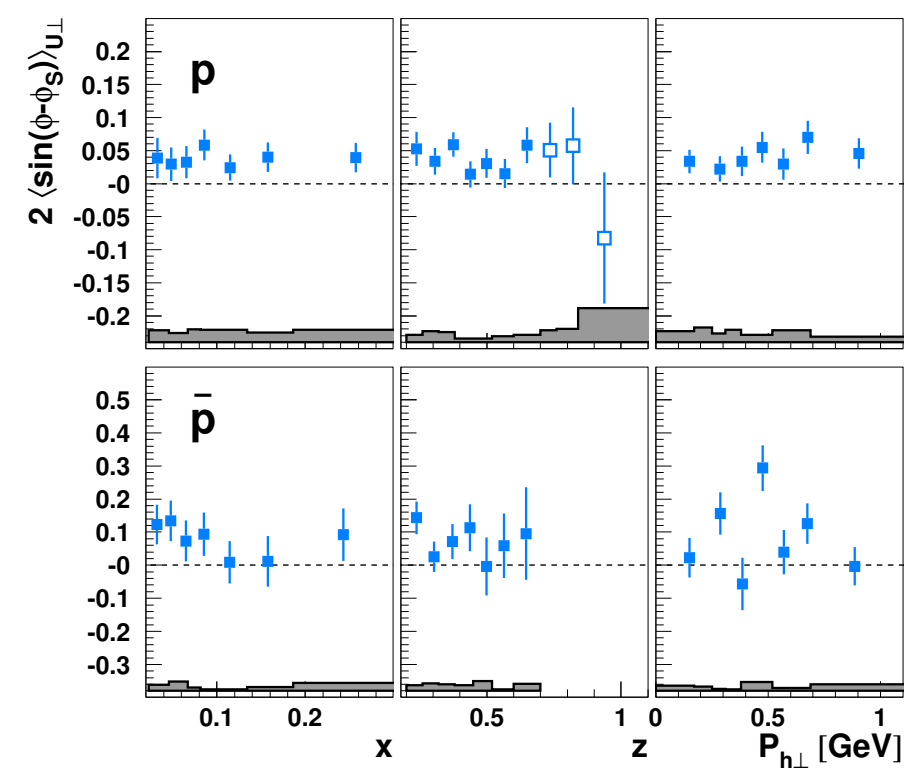

Figure 16. Sivers SFA for protons (upper row) and antiprotons (lower row) presented either in bins of $x, z$, or $P_{h \perp}$. Data at large values of $z$, marked by open points in the $z$ projection, are not included in the other projections (no such high- $z$ points are available for antiprotons due to a lack of precision). Systematic uncertainties are given as bands, not including the additional scale uncertainty of $7.3 \%$ due to the precision of the target-polarization determination.

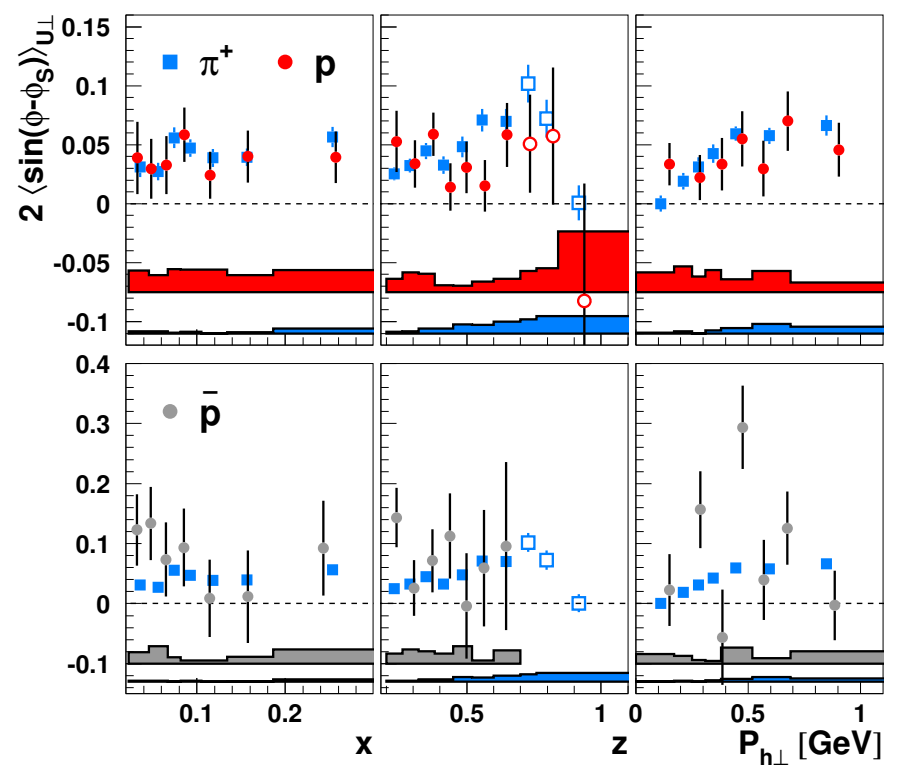

Figure 17. Comparison of Sivers SFA for positive pions and protons (upper plot) or antiprotons (lower plot) presented either in bins of $x, z$, or $P_{h \perp}$. Data at large values of $z$, marked by open points in the $z$ projection, are not included in the other projections (no such high- $z$ points are available for antiprotons due to a lack of precision). Systematic uncertainties are given as bands, not including the additional scale uncertainty of $7.3 \%$ due to the precision of the target-polarization determination. 


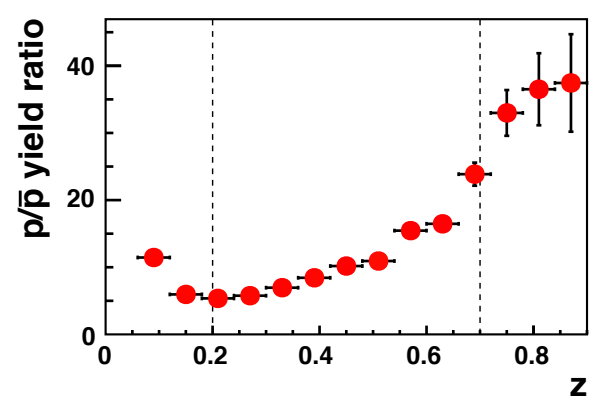

Figure 18. Ratio of raw proton to antiproton yields at HERMES as a function of $z$. The bin boundaries for the semi-inclusive DIS range are marked by dashed lines. The ratio exhibits a clear rise towards very low $z$, which might indicate the onset of significant target-fragmentation contributions, excluded in the data sample used by the minimum- $z$ requirement of 0.2 .

scattering, which exhibits a positive Sivers asymmetry. The recoiling target fragments are thus expected to exhibit a Sivers asymmetry of opposite sign. As the proton Sivers asymmetry is positive, it appears less likely that those protons came from the fragmenting target. All these features are, however, also not sufficient to establish that the protons and antiprotons are dominantly produced in the hadronization of the current-quark jet, which needs to be kept in mind when interpreting the results in such framework.

\subsection{The vanishing signals for the pretzelosity function}

The chiral-odd pretzelosity distribution, $h_{1 \mathrm{~T}}^{\perp, q}\left(x, \mathbf{p}_{T}^{2}\right)$, provides information about the nonspherical shape of transversely polarized protons in momentum space caused by significant contributions from orbital angular momentum to a quadrupole modulation of the parton distributions [52]. It can be accessed coupled to the chiral-odd Collins fragmentation function in semi-inclusive deep-inelastic scattering through the $\sin \left(3 \phi-\phi_{S}\right)$ modulation of the cross section. So far, only measurements of this amplitude for charged pions using a transversely polarized ${ }^{3} \mathrm{He}$ target by the Jefferson Lab Hall A Collaboration [55] and for unidentified hadrons using a transversely polarized $\mathrm{NH}_{3}$ target by the COMPASS Collaboration [56] have been published and found to be consistent with zero. In a combination of the data from Jefferson Lab Hall A with preliminary data from both the COMPASS and HERMES collaborations as well as the Collins fragmentation function from a phenomenological analysis [111], $h_{1 \mathrm{~T}}^{\perp, q}\left(x, \mathbf{p}_{T}^{2}\right)$ was extracted both for up and down quarks and found to be consistent with zero albeit within large uncertainties [165].

The underlying transverse-momentum convolution in eq. (2.7) involves a weight that is expected to scale with $P_{h \perp}^{3}$. As relatively low transverse momenta are observed, $\left\langle P_{h \perp}\right\rangle<$ $1 \mathrm{GeV}$, the amplitude of the $\sin \left(3 \phi-\phi_{S}\right)$ modulation is suppressed with respect to, e.g., the Collins amplitude, which also involves a convolution of a chiral-odd parton distribution with the Collins fragmentation function, but which scales with $P_{h \perp}$.

In this analysis, the $2\left\langle\sin \left(3 \phi-\phi_{S}\right) / \epsilon\right\rangle_{\mathrm{U} \perp}^{h}$ amplitudes, shown in figure 19 for charged mesons and in figure 20 for neutral pions as well as for (anti)protons, are found to be consistent with zero. There is a hint of a small negative amplitude for negative pions that is, however, statistically not sufficiently significant to claim a non-vanishing pretzelosity. 

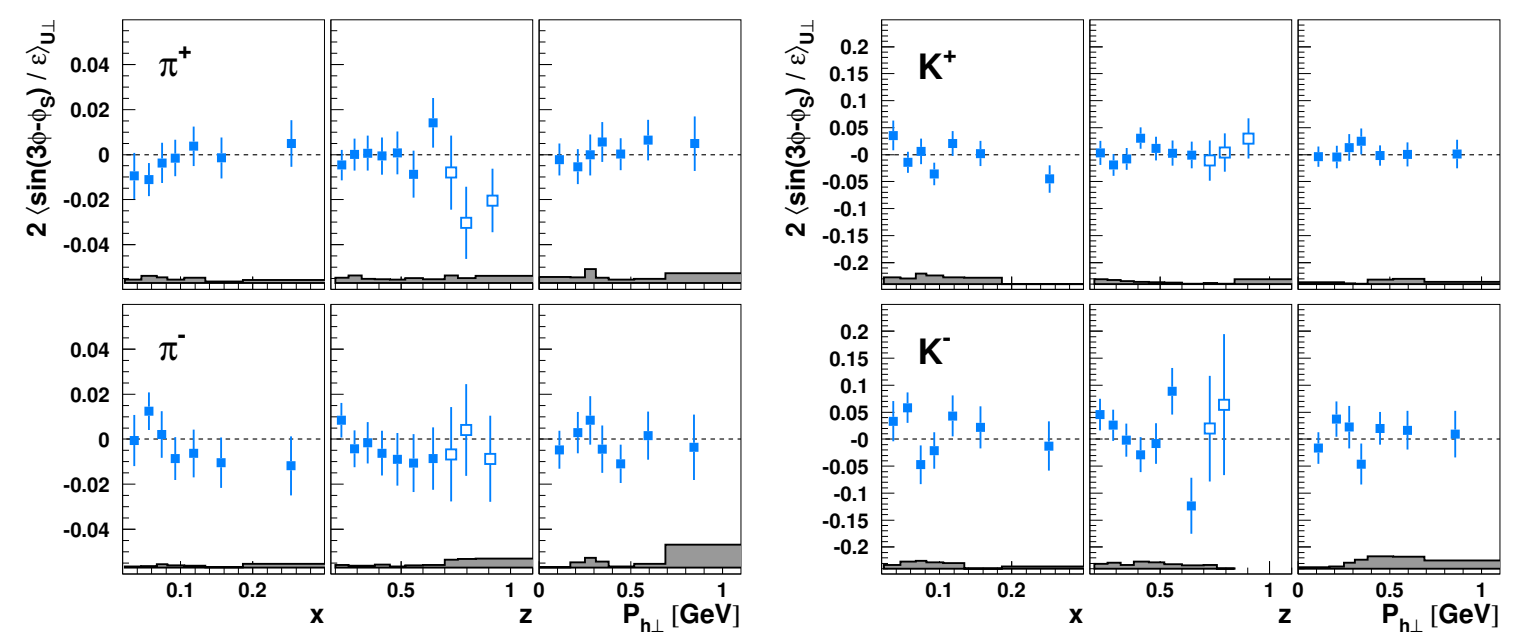

Figure 19. Pretzelosity SFA for charged mesons (left: pions; right: kaons) presented either in bins of $x, z$, or $P_{h \perp}$. Data at large values of $z$, marked by open points in the $z$ projection, are not included in the other projections. Systematic uncertainties are given as bands, not including the additional scale uncertainty of $7.3 \%$ due to the precision of the target-polarization determination.
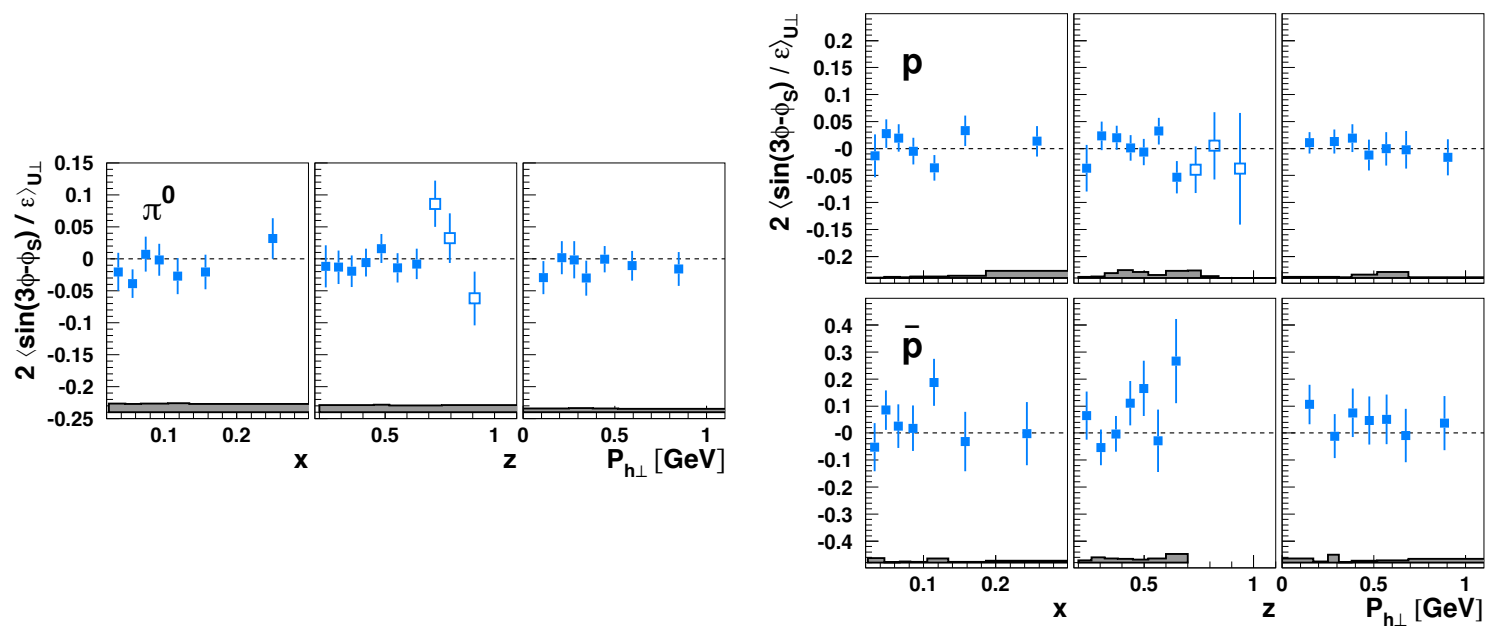

Figure 20. Pretzelosity SFA for $\pi^{0}$ (left), protons, and antiprotons (right) presented either in bins of $x, z$, or $P_{h \perp}$. Data at large values of $z$, marked by open points in the $z$ projection, are not included in the other projections (no such high- $z$ points are available for antiprotons due to a lack of precision). Systematic uncertainties are given as bands, not including the additional scale uncertainty of $7.3 \%$ due to the precision of the target-polarization determination.

As noted before, the pretzelosity amplitudes are expected to be suppressed. Cancelations, e.g., from the Collins function that changes sign for favored and disfavored fragmentation, might also contribute to the vanishing signal. Model calculations thus predict in general small asymmetries below 0.01 (see, e.g., ref. [60]), beyond the precision of this measurement. 

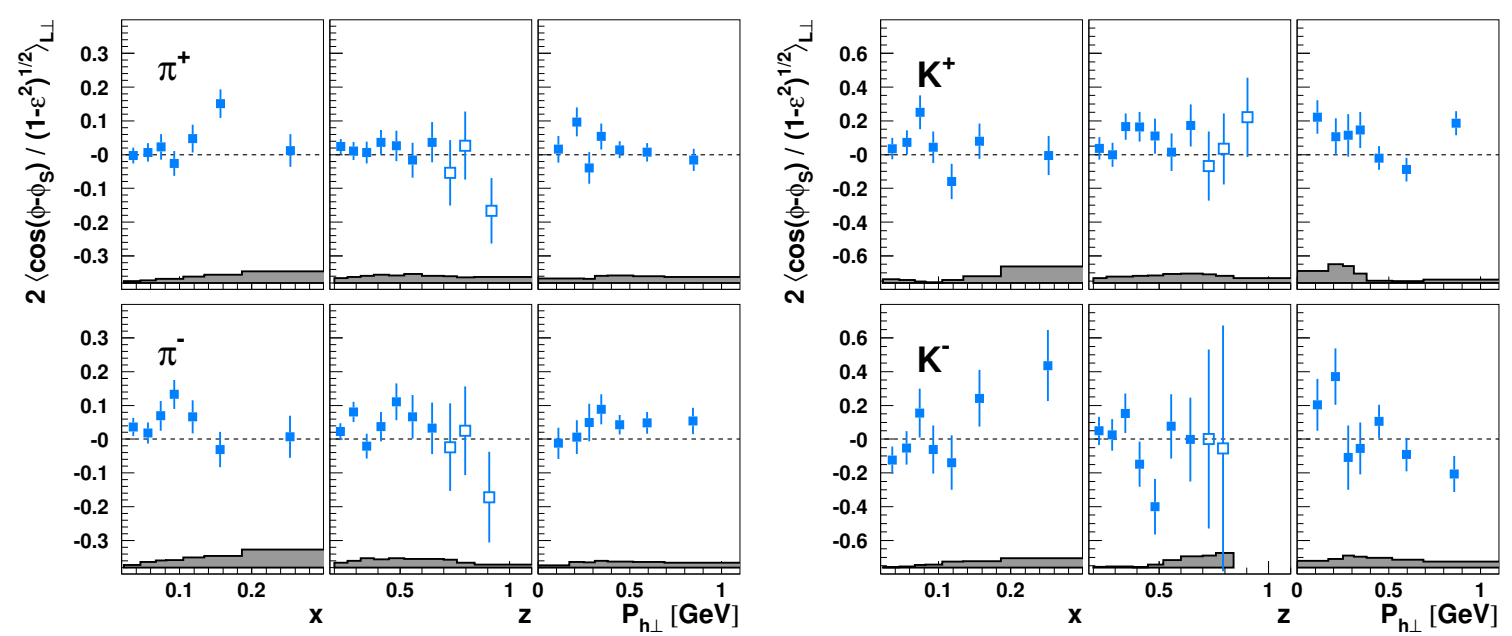

Figure 21. The $2\left\langle\cos \left(\phi-\phi_{S}\right) / \sqrt{1-\epsilon^{2}}\right\rangle_{\mathrm{L} \perp}^{h}$ amplitudes for charged mesons (left: pions; right: kaons) presented either in bins of $x, z$, or $P_{h \perp}$. Data at large values of $z$, marked by open points in the $z$ projection, are not included in the other projections. Systematic uncertainties are given as bands, not including the additional scale uncertainty of $8.0 \%$ due to the precision in the determination of the target and beam polarizations.

\subsection{Signals for the worm-gear (II) distribution $g_{1 \mathrm{~T}}^{q}\left(x, \mathbf{p}_{T}^{2}\right)$}

The naive- $T$-even and chiral-even worm-gear (II) distribution $g_{1 \mathrm{~T}}^{q}\left(x, \mathbf{p}_{T}^{2}\right)$ is unique in the sense that it is the only TMD that vanishes when integrating over $\mathbf{p}_{T}$ but neither entails nor is affected by final-state interactions. At leading twist, this TMD cannot contribute to naive- $T$-odd effects that cause single-spin asymmetries. Its spin-orbit correlation, $\lambda S_{T}^{i} p_{T}^{i}$, involves a common product of the helicity of the struck quark and the transverse spin direction of the nucleon. In combination with the selection of quarks with a certain helicity by a longitudinally polarized lepton beam, the worm-gear (II) distribution $g_{1 \mathrm{~T}}^{q}\left(x, \mathbf{p}_{T}^{2}\right)$ can be related to the $\cos \left(\phi-\phi_{S}\right)$ modulation of the double-spin asymmetry in the scattering of longitudinally polarized leptons by transversely polarized nucleons.

This $\cos \left(\phi-\phi_{S}\right)$ modulation provides a leading-twist signal for the worm-gear (II) distribution $g_{1 \mathrm{~T}}^{q}\left(x, \mathbf{p}_{T}^{2}\right)$ in combination with the spin-independent fragmentation function $D_{1}^{q \rightarrow h}\left(z, z^{2} \mathbf{k}_{T}^{2}\right)$ [cf. eq. (2.10)]. As such it is not additionally suppressed in the asymmetry amplitude by the relative magnitude of $H_{1}^{\perp, q \rightarrow h}\left(z, z^{2} \mathbf{k}_{T}^{2}\right)$ compared to $D_{1}^{q \rightarrow h}\left(z, z^{2} \mathbf{k}_{T}^{2}\right)$.

In figures 21 and 22 , the $2\left\langle\cos \left(\phi-\phi_{S}\right) / \sqrt{1-\epsilon^{2}}\right\rangle_{\mathrm{L} \perp}^{h}$ Fourier amplitudes of the doublespin asymmetry $A_{\mathrm{L} \perp}^{h}$ are presented for pions, charged kaons, as well as for (anti)protons. As a consequence of the relatively small degree of polarization of the HERA lepton beam during the years 2002-2005, the statistical uncertainties are generally larger than those for the Fourier amplitudes of the transverse single-spin asymmetry $A_{\mathrm{U} \perp}^{h}$.

For positively charged pions, non-vanishing $2\left\langle\cos \left(\phi-\phi_{S}\right) / \sqrt{1-\epsilon^{2}}\right\rangle_{\mathrm{L} \perp}^{h}$ Fourier amplitudes are extracted, providing an indication for a non-vanishing worm-gear (II) distribution $g_{1 \mathrm{~T}}^{q}\left(x, \mathbf{p}_{T}^{2}\right)$. Results for $\pi^{-}$and $K^{+}$are inconsistent with zero at $90 \%$ but not at $95 \%$ confidence level.

When comparing the meson results to the Sivers asymmetries, which also involve only the ordinary $D_{1}^{q}\left(z, z^{2} \mathbf{k}_{T}^{2}\right)$ fragmentation function and are thus easier to interpret in terms 

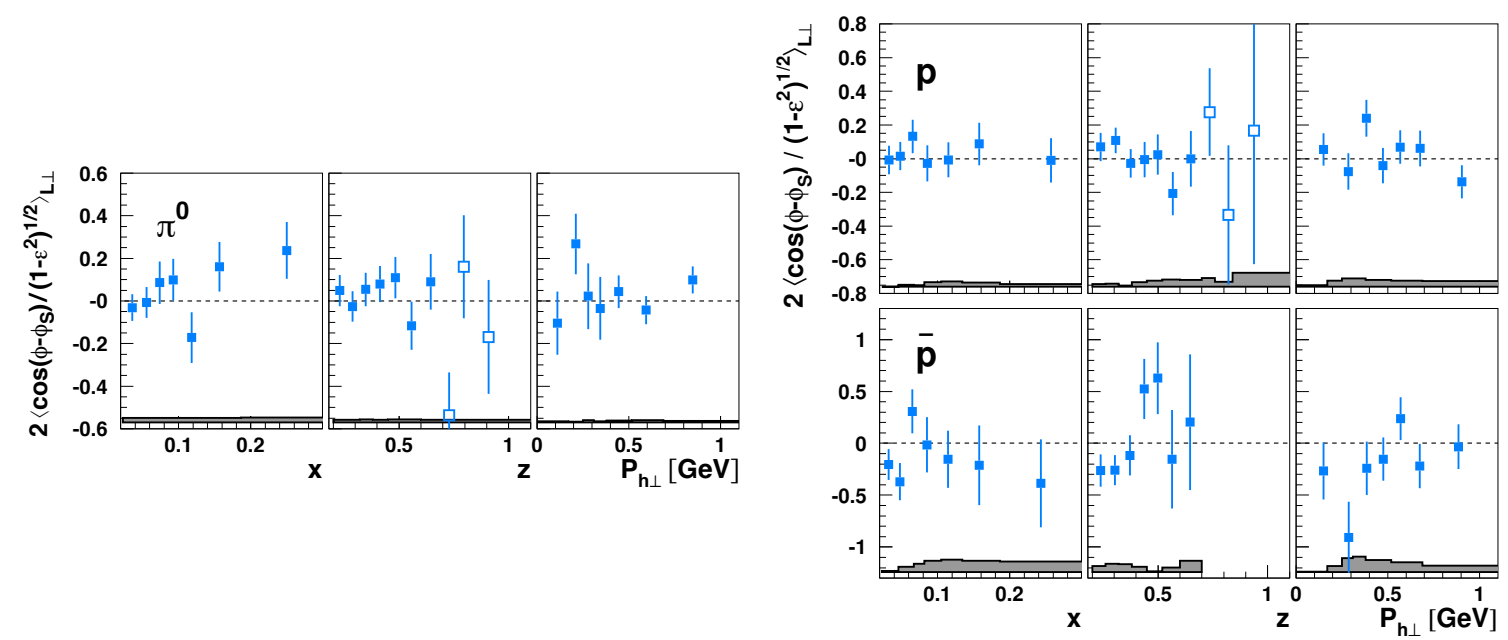

Figure 22. The $2\left\langle\cos \left(\phi-\phi_{S}\right) / \sqrt{1-\epsilon^{2}}\right\rangle_{\mathrm{L} \perp}^{h}$ amplitudes for $\pi^{0}$ (left), protons, and antiprotons (right) presented either in bins of $x, z$, or $P_{h \perp}$. Data at large values of $z$, marked by open points in the $z$ projection, are not included in the other projections (no such high- $z$ points are available for antiprotons due to a lack of precision). Systematic uncertainties are given as bands, not including the additional scale uncertainty of $8.0 \%$ due to the precision in the determination of the target and beam polarizations.

of separate quark-flavor contributions, a similar picture becomes apparent: mainly the positively charged mesons exhibits a (positive) $2\left\langle\cos \left(\phi-\phi_{S}\right) / \sqrt{1-\epsilon^{2}}\right\rangle_{\mathrm{L} \perp}$ amplitude. In analogy to the Sivers discussion, taking into account the additional minus sign in the Sivers convolution (2.6) compared to (2.10), the data suggest that $g_{1 \mathrm{~T}}^{u}\left(x, \mathbf{p}_{T}^{2}\right)$ is positive.

However, all of the above discussion is merely qualitative in view of the large uncertainties of this measurement. In that respect, it should be emphasized that tremendous progress has been made predicting $g_{1 \mathrm{~T}}^{q}\left(x, \mathbf{p}_{T}^{2}\right)$ based on models (e.g., refs. $\left.[60,166]\right)$ and by now also lattice-QCD calculations $[167,168]$. A common thread among the calculations is a positive $g_{1 \mathrm{~T}}^{u}\left(x, \mathbf{p}_{T}^{2}\right)$ and a negative $g_{1 \mathrm{~T}}^{d}\left(x, \mathbf{p}_{T}^{2}\right)$, not at variance with the above discussion. For example, the calculation in ref. [60] — based on the light-cone constituent-quark model - predicts positive $2\left\langle\cos \left(\phi-\phi_{S}\right)\right\rangle_{\mathrm{LT}}$ Fourier amplitudes for charged pions of the order of 2-3\%, larger for $\pi^{+}$than for $\pi^{-}$, which qualitatively agrees with the results presented here. The COMPASS experiment found positive $2\left\langle\cos \left(\phi-\phi_{S}\right)\right\rangle_{\mathrm{LT}}$ Fourier amplitudes for unidentified charged hadrons [56]. The results by the Jefferson Lab Hall A Collaboration [66] using a transversely polarized ${ }^{3} \mathrm{He}$ target, which essentially can be regarded as a neutron target, show a large positive asymmetry for $\pi^{-}$while the $\pi^{+}$asymmetry is consistent with zero, also consistent with the model predictions.

\subsection{The subleading-twist SSA and DSA Fourier amplitudes}

Four modulations contributing to the cross sections (2.1) involving transverse target polarization (two of which require in addition longitudinal beam polarization) vanish at twist-2 level and thus involve either twist-3 distribution or fragmentation functions, as detailed in section 2.2.5. As such they offer a way to constrain multi-parton correlations, while on the 

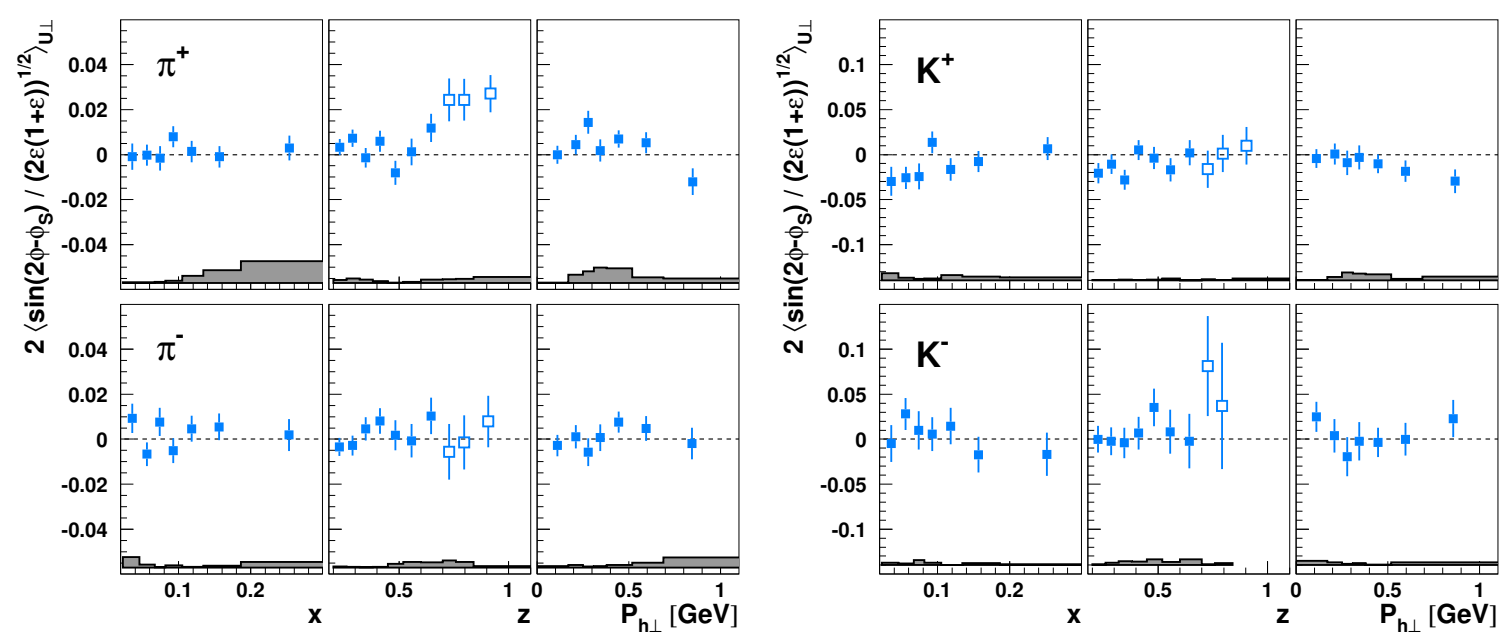

Figure 23. The $2\left\langle\sin \left(2 \phi-\phi_{S}\right) / \sqrt{2 \epsilon(1+\epsilon)}\right\rangle_{\mathrm{U} \perp}^{h}$ amplitudes for charged mesons (left: pions; right: kaons) presented either in bins of $x, z$, or $P_{h \perp}$. Data at large values of $z$, marked by open points in the $z$ projection, are not included in the other projections. Systematic uncertainties are given as bands, not including the additional scale uncertainty of $7.3 \%$ due to the precision of the targetpolarization determination.

other hand being expected to be small as formally suppressed by $M / Q$. Interpretation of those modulations is hampered by the multitude of twist-3 functions contributing, often lacking clear guidance from phenomenology. Wandzura-Wilczek-type approximations [64] help to reduce the number of terms, but have their own limitations. For example, the clearly non-vanishing beam-helicity asymmetry in, e.g., ref. [72] challenges the WandzuraWilczek-type approximation, the latter predicting asymmetries identical to zero.

The results presented below constitute the first measurement of those subleading-twist Fourier amplitudes in semi-inclusive deep-inelastic scattering by transversely polarized protons.

The $2\left\langle\sin \left(2 \phi-\phi_{S}\right) / \sqrt{2 \epsilon(1+\epsilon)}\right\rangle_{\mathrm{U} \perp}^{h}$ Fourier amplitudes are found to be mostly consistent with zero as shown in figures 23 and 24 . Within the semi-inclusive DIS kinematic range of the measurement, they are consistent with zero at $95 \%$ confidence level for all hadrons and only at $90 \%$ confidence level inconsistent with zero for antiprotons (cf. table 9).

Besides the suppression from being a twist-3 observable, the Fourier amplitude of the $\sin \left(2 \phi-\phi_{S}\right)$ modulation is subject to a $P_{h \perp}$ suppression arising through the transversemomentum convolution. This is similar to what was discussed for pretzelosity in section 4.3. However, in comparison to, e.g., the Collins and Sivers modulations, it is only one additional power of $P_{h \perp}$ and not two. Looking at the $K^{+}$results, which hint a slightly negative modulation at low $x$, non-vanishing asymmetries are indeed only visible at large $P_{h \perp}$, where such $P_{h \perp}$ suppression should die out.

Small asymmetries on the sub-percent level consistent with these data are predicted for pions in Wandzura-Wilczek-type approximations [64], in which only the terms involving the twist-3 TMDs $f_{\mathrm{T}}^{\perp, q}, h_{\mathrm{T}}^{q}$, and $h_{\mathrm{T}}^{\perp, q}$ give contributions. Similarly, a calculation based on a spectator-diquark model for those three TMDs results again in only a small $\sin \left(2 \phi-\phi_{S}\right)$ modulation consistent with the measurement presented here [169]. 

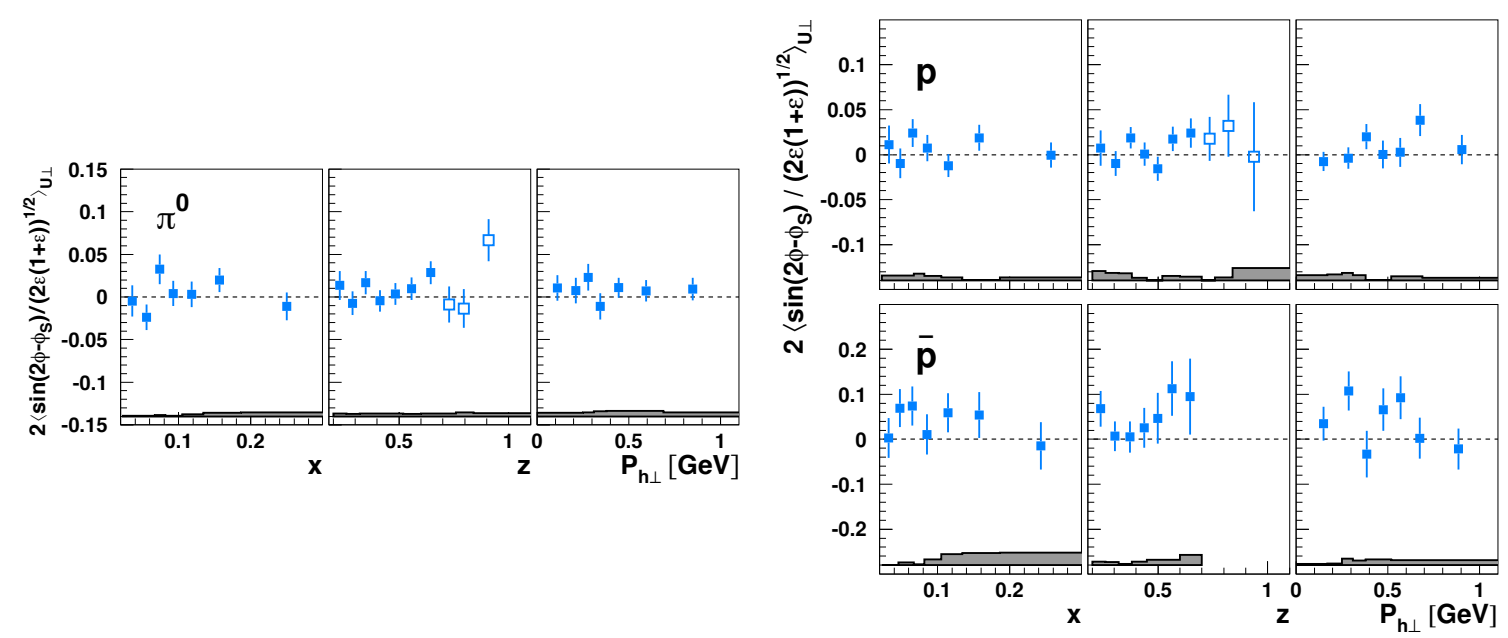

Figure 24. The $2\left\langle\sin \left(2 \phi-\phi_{S}\right) / \sqrt{2 \epsilon(1+\epsilon)}\right\rangle_{\mathrm{U} \perp}^{h}$ amplitudes for $\pi^{0}$ (left), protons, and antiprotons (right) presented either in bins of $x, z$, or $P_{h \perp}$. Data at large values of $z$, marked by open points in the $z$ projection, are not included in the other projections (no such high- $z$ points are available for antiprotons due to a lack of precision). Systematic uncertainties are given as bands, not including the additional scale uncertainty of $7.3 \%$ due to the precision of the target-polarization determination.

In the semi-exclusive region of $z>0.7$, a positive $2\left\langle\sin \left(2 \phi-\phi_{S}\right) / \sqrt{2 \epsilon(1+\epsilon)}\right\rangle_{\mathrm{U} \perp}$ Fourier amplitude on the order of 0.02 is extracted for positive pions. In general, the interpretation of asymmetries in this region in terms of TMDs is to be taken with caution; still, an attempt is provided below. From the various terms contributing to the related structure function in eq. (2.12), three are increasingly suppressed with increasing $z$. The very first term reduces in the Wandzura-Wilczek-type approximation [64] to the Sivers effect, albeit with the opposite sign compared to the leading-twist Sivers asymmetry. The measured Sivers asymmetries are indeed large at high $z$. However, as they exhibit the same sign as the $2\left\langle\sin \left(2 \phi-\phi_{S}\right) / \sqrt{2 \epsilon(1+\epsilon)}\right\rangle_{\mathrm{U} \perp}$ Fourier amplitudes, either the Wandzura-Wilczektype approximation predicts the wrong sign (and thus appears to fail) or the positive $2\left\langle\sin \left(2 \phi-\phi_{S}\right) / \sqrt{2 \epsilon(1+\epsilon)}\right\rangle_{\mathrm{U} \perp}$ Fourier amplitudes in the high- $z$ region need to be attributed to other contributions. A possibility could be the second contribution in eq. (2.12) that is not formally suppressed for large values of $z$ : the combined contribution of $h_{\mathrm{T}}^{q}+h_{\mathrm{T}}^{\perp, q}$ coupled to the Collins fragmentation function. In the Wandzura-Wilczek-type approximation it is related to pretzelosity, but generally found to be very small $[64,169]$. There is some similarity of the large- $z$ behavior of the $2\left\langle\sin \left(2 \phi-\phi_{S}\right) / \sqrt{2 \epsilon(1+\epsilon)}\right\rangle_{\mathrm{U} \perp}^{\pi^{+}}$to that of the $2\left\langle\sin \left(2 \phi+\phi_{S}\right) / \epsilon\right\rangle_{\mathrm{U} \perp}^{\pi^{+}}$Fourier amplitude discussed further below (cf. section 4.6). As both modulations receive the same cross-section contribution from the longitudinal targetpolarization component, the source for the non-vanishing asymmetries at large $z$ might indeed stem from a $2\langle\sin (2 \phi)\rangle_{\mathrm{UL}}^{\pi^{+}}$Fourier amplitude of the longitudinal SSA. Unfortunately, not much is known about the latter amplitude in the kinematic regime of this measurement. HERMES data for the related $2\langle\sin (2 \phi)\rangle_{\mathrm{U} \|}^{h}$ Fourier amplitude for charged pions [67] are consistent with zero when integrated over the semi-inclusive $z$ range of $0.2<z<0.7$, with- 

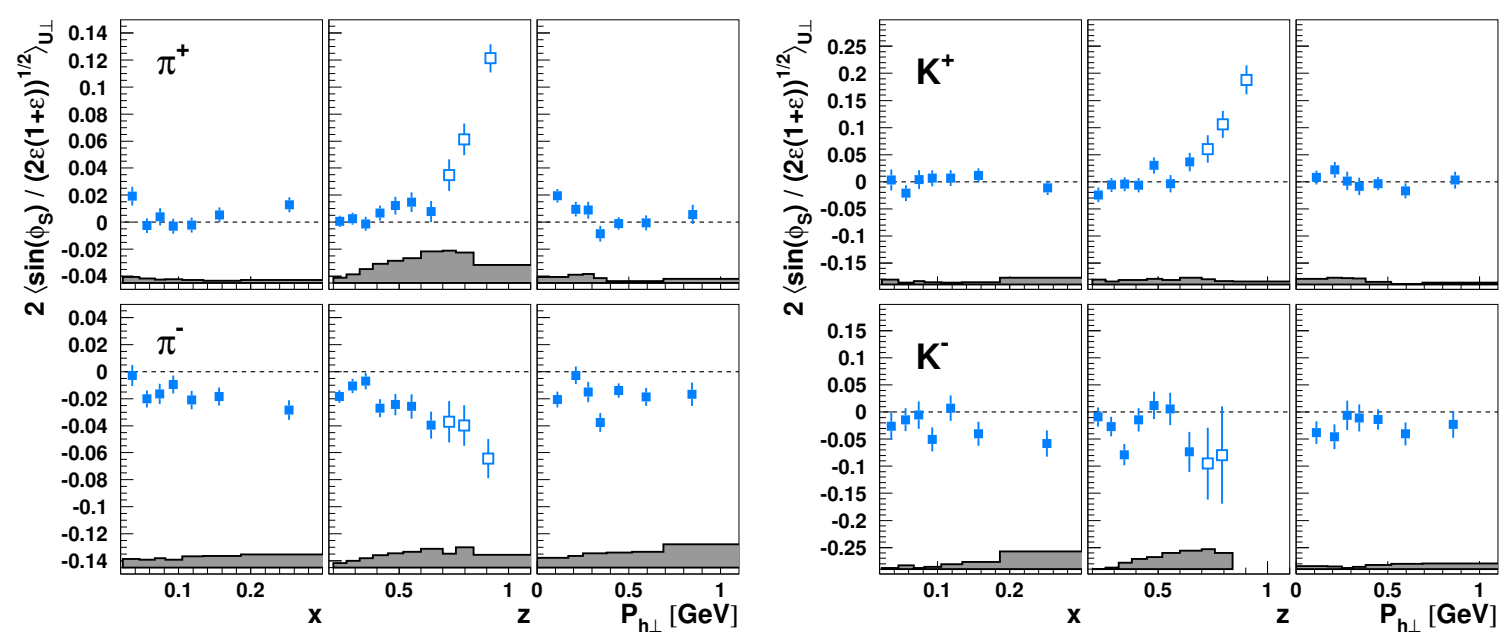

Figure 25. The $2\left\langle\sin \left(\phi_{S}\right) / \sqrt{2 \epsilon(1+\epsilon)}\right\rangle_{\mathrm{U} \perp}^{h}$ amplitudes for charged mesons (left: pions; right: kaons) presented either in bins of $x, z$, or $P_{h \perp}$. Data at large values of $z$, marked by open points in the $z$ projection, are not included in the other projections. Systematic uncertainties are given as bands, not including the additional scale uncertainty of $7.3 \%$ due to the precision of the target-polarization determination.

out presenting data binned in $z$ or for $z>0.7$. Likewise, preliminary COMPASS data, both for the semi-inclusive $z$ region and for large $z$, do not exhibit a sizable $2\langle\sin (2 \phi)\rangle_{\mathrm{U} \|}^{h}$ asymmetry [170]. Only the CLAS collaboration reported non-vanishing $2\langle\sin (2 \phi)\rangle_{\mathrm{U} \|}^{h}$ asymmetry amplitudes for charged pions [171], however, not for the $z>0.7$ range considered here. In contrast to the earlier HERMES measurement of $2\langle\sin (2 \phi)\rangle_{\mathrm{U} \|}^{h}$, the CLAS data are on average at larger $z$ since they are integrated over the range $0.4<z<0.7$. Thus, the non-zero CLAS data might be a hint of an increase in magnitude of these asymmetry amplitudes with increasing $z$. On the other hand, the negative values of these asymmetry amplitudes are not compatible with the positive $2\left\langle\sin \left(2 \phi-\phi_{S}\right) / \sqrt{2 \epsilon(1+\epsilon)}\right\rangle_{\mathrm{U} \perp}^{\pi^{+}}$amplitudes presented here. Last but not least, positive $\sin \left(2 \phi-\phi_{S}\right)$ modulations have been observed in exclusive $\pi^{+}$electroproduction off transversely polarized protons [172], which suggests a smooth transition from the semi-exclusive high- $z$ region studied here to exclusive $\pi^{+}$ production.

One of the more striking results of this analysis is the observation of large subleadingtwist $2\left\langle\sin \left(\phi_{S}\right) / \sqrt{2 \epsilon(1+\epsilon)}\right\rangle_{\mathrm{U} \perp}^{h}$ Fourier amplitudes. In particular, they provide the largest twist-3 signal in this measurement. They surprise also with a large kinematic dependence as visible in figure 25, where they are shown for charged mesons. In the semi-inclusive deep-inelastic scattering region, mainly the Fourier amplitudes for negative mesons are significantly different from zero, being of order -0.02 . The three-dimensional binning, depicted in figure 26 for the $\pi^{-}$, reveals that those non-vanishing asymmetries stem predominantly from the large- $x$ and large- $z$ region, where they reach even larger magnitudes. The amplitudes clearly rise with $z$ for charged pions and positive kaons. The precision for $\mathrm{K}^{-}$ and neutral pions in that region is insufficient for drawing a strong conclusion, though also here an increase in magnitude with $z$ is hinted. A noteworthy characteristic of the results 


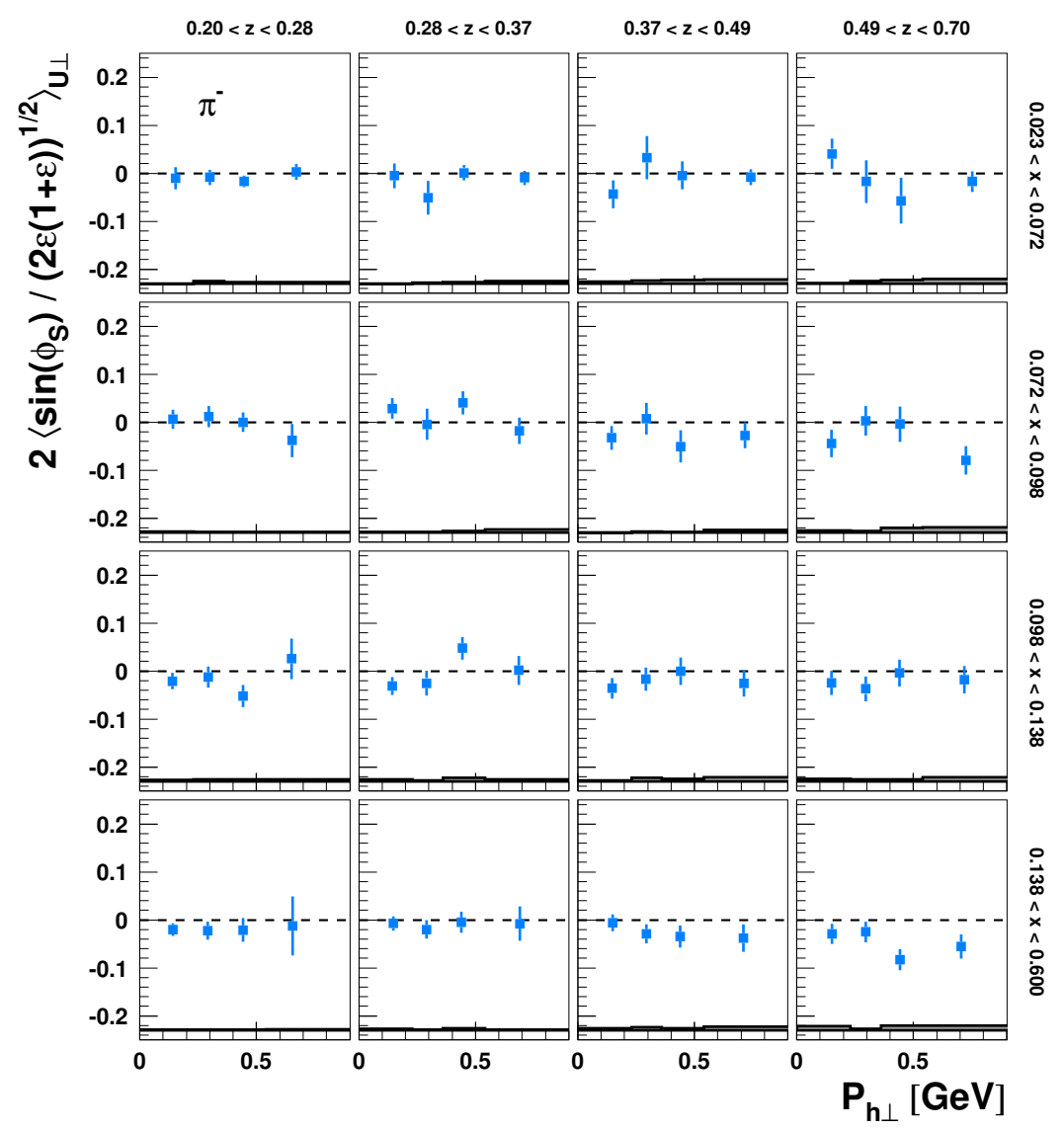

Figure 26. The $2\left\langle\sin \left(\phi_{S}\right) / \sqrt{2 \epsilon(1+\epsilon)}\right\rangle_{\mathrm{U} \perp}$ Fourier amplitudes for $\pi^{-}$extracted simultaneously in bins of $x, z$, and $P_{h \perp}$, presented as a function of $P_{h \perp}$. Systematic uncertainties are given as bands, not including the additional scale uncertainty of $7.3 \%$ due to the precision of the target-polarization determination.

is the clearly opposite sign for the $\pi^{-}$results compared to both $\pi^{+}$and $K^{+}$, reminiscent of what is observed for the Collins asymmetries.

The Fourier amplitudes of the $\sin \left(\phi_{S}\right)$ modulations are related to subleading-twist cross-section contributions (cf. eq. (2.13)). As such it is interesting to explore the $Q^{2}$ dependence of this azimuthal asymmetry. Because $x$ and $Q^{2}$ are highly correlated, a onedimensional binning in $Q^{2}$ mixes effects from the twist-3 suppression with the inherent $x$ dependence of the asymmetry. Therefore, an approach employed already in the previous HERMES Collins and Sivers publications $[29,40]$ has been adopted here that splits each $x$ bin into the two regions of $Q^{2}$ : below and above the average $Q^{2}$ of each $x$ bin. The resulting $\pi^{-}$CSA amplitudes are shown in figure 27. A hint of a suppression is visible for the regions of larger $Q^{2}$, though not very pronounced, which might be a consequence of the relatively small lever arm in $Q^{2}$ as apparent from the difference in average $Q^{2}$ for the two regions, plotted in the bottom panel of the figure. 


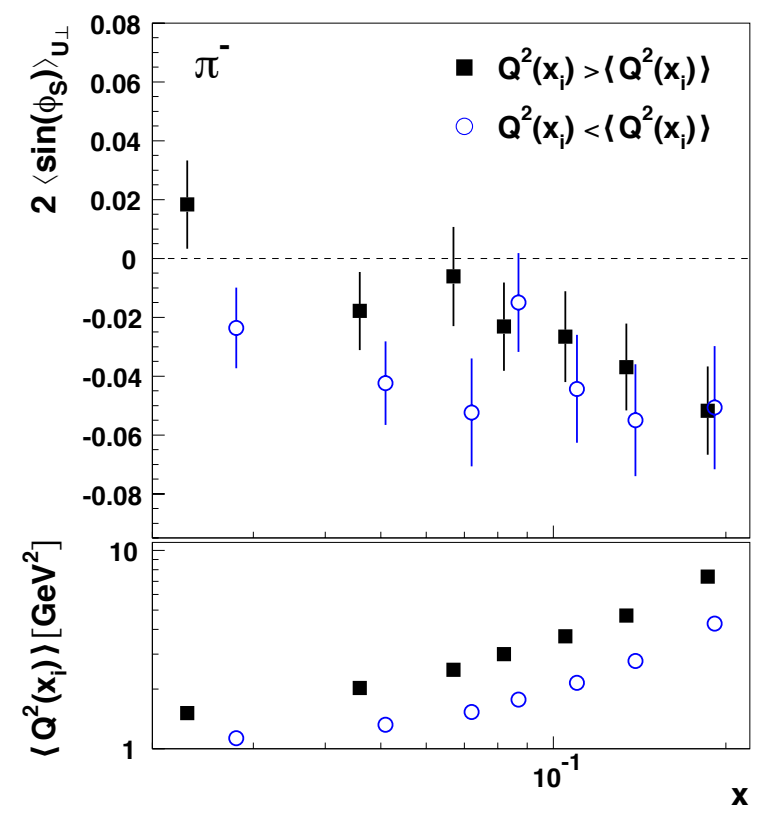

Figure 27. The $2\left\langle\sin \left(\phi_{S}\right)\right\rangle_{\mathrm{U} \perp}^{h} \mathrm{CSA}$ amplitudes for $\pi^{-}$as a function of $x$. The $Q^{2}$ region for each bin was divided into the two regions above (squares) and below (circles) the average $Q^{2}$ of that bin. The average $Q^{2}$ is given in the bottom for all bins separately for the two $Q^{2}$ regions. The error bars represent statistical uncertainties only.

The structure function $F_{\mathrm{UT}}^{\sin \left(\phi_{S}\right)}$ is of particular interest as it is the only contribution to the cross section $\sigma_{\mathrm{UT}}^{h}$ that survives integration over transverse hadron momentum:

$$
F_{\mathrm{UT}}^{\sin \left(\phi_{S}\right)}\left(x, Q^{2}, z\right)=\int d^{2} \mathbf{P}_{h \perp} F_{\mathrm{UT}}^{\sin \left(\phi_{S}\right)}\left(x, Q^{2}, z, P_{h \perp}\right)=-x \frac{2 M_{h}}{Q} \sum_{q} e_{q}^{2} h_{1}^{q} \frac{\tilde{H}^{q}(z)}{z} .
$$

It thus provides, in principle, sensitivity to the transversity distribution without involving a convolution over intrinsic transverse momenta [73]. In addition, the modulation does not necessarily have to vanish in the limit of $P_{h \perp}$ going to zero. Another rather interesting aspect of the $\sin \left(\phi_{S}\right)$ modulation - as pointed out already in section 2.2 .5 - is the fact that the inclusive analogue, i.e., summing over all final-state hadrons and integrating over their four-momenta, must vanish in the one-photon-exchange approximation, which was tested at HERMES to the $10^{-3}$ level [75].

A serious experimental drawback in using the relation (4.1) to extract transversity could be the systematic effect arising from the usually incomplete integration over $\mathbf{P}_{h \perp}$ due to limitations in the geometric acceptance or kinematic requirements in experiments. Furthermore, a current drawback of such measurement is the lack of knowledge about the interaction-dependent fragmentation function $\tilde{H}^{q}(z)$. However, it has been shown that the latter, the Collins fragmentation function, as well as the collinear twist-3 fragmentation function that is suspected to cause the transverse-spin asymmetries in inclusive pion production in single-polarized proton-proton collisions are related $[173,174]$. This may explain the similar qualitative behavior of the Collins asymmetries and of the $2\left\langle\sin \left(\phi_{S}\right)\right\rangle_{\mathrm{U} \perp}^{\pi}$ Fourier amplitudes. 

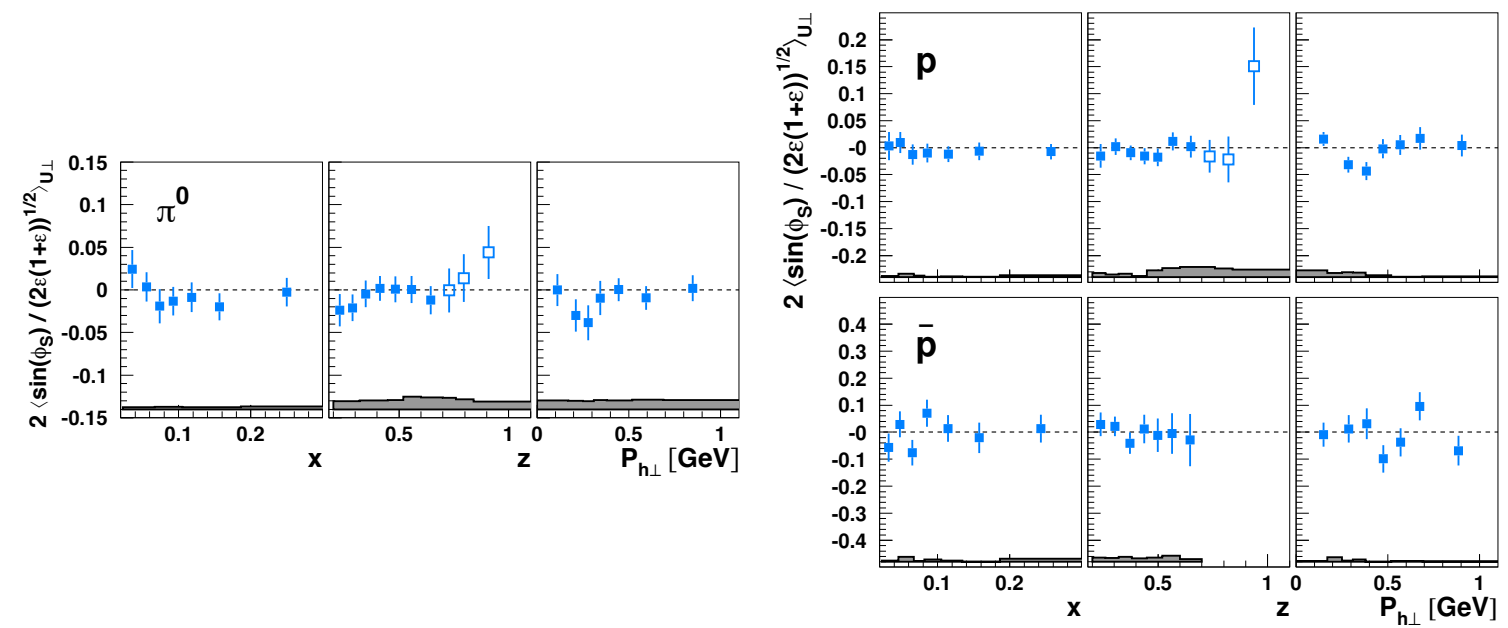

Figure 28. The $2\left\langle\sin \left(\phi_{S}\right) / \sqrt{2 \epsilon(1+\epsilon)}\right\rangle_{\mathrm{U} \perp}^{h}$ amplitudes for $\pi^{0}$ (left), protons, and antiprotons (right) presented either in bins of $x, z$, or $P_{h \perp}$. Data at large values of $z$, marked by open points in the $z$ projection, are not included in the other projections (no such high- $z$ points are available for antiprotons due to a lack of precision). Systematic uncertainties are given as bands, not including the additional scale uncertainty of $7.3 \%$ due to the precision of the target-polarization determination.

The relation to the Collins effect might also explain why the results for protons and antiprotons are consistent with zero, as shown in figure 28 (where also the vanishing signal for $\pi^{0}$ is presented). As novel spin-dependent fragmentation is involved, it is reasonable to expect a fundamental difference for production of spin-0 versus spin- $\frac{1}{2}$ hadrons.

The vanishing effect for protons and the negative asymmetry for $\pi^{-}$also disfavor a sizable contribution of $f_{\mathrm{T}}^{q} D_{1}^{q \rightarrow h}$ in eq. (2.13) - which can be related to the Sivers effect in the Wandzura-Wilczek-type approximation - being in conflict with the behavior of the Sivers asymmetry for those hadrons. Furthermore, $f_{\mathrm{T}}^{q}$ has to fulfill the sum rule $\int \mathrm{d}^{2} \mathbf{p}_{T} f_{\mathrm{T}}^{q}\left(x, \mathbf{p}_{T}^{2}\right)=0$, which poses a problem when using currently available parameterization for the Sivers function in the Wandzura-Wilczek-type approximation for $f_{\mathrm{T}}^{q}$ because they are violating the sum rule. For that reason, it is not further considered here.

Staying within the Wandzura-Wilczek-type approximation, from the remaining terms in eq. (2.13) contributing to the $2\left\langle\sin \left(\phi_{S}\right)\right\rangle_{\mathrm{UT}}^{\pi}$ Fourier amplitude only the ones involving the Collins fragmentation function survive. The combined contribution involves $-x\left(h_{\mathrm{T}}^{q}-\right.$ $\left.h_{\mathrm{T}}^{\perp, q}\right) \stackrel{\mathrm{WW}}{=} h_{1}^{q}$ and thus the product of transversity and the Collins fragmentation function. As in the above discussion of the $\tilde{H}^{q}(z)$ contribution, this might explain the qualitative similarity of the charged-pion Collins and $2\left\langle\sin \left(\phi_{S}\right)\right\rangle_{\mathrm{U} \perp}^{\pi}$ Fourier amplitudes.

In contrast to the $2\left\langle\sin \left(2 \phi-\phi_{S}\right)\right\rangle_{\mathrm{U} \perp}^{h}$ Fourier amplitude, there is no additional term contributing through the longitudinal target-polarization component. As a consequence, the $2\left\langle\sin \left(\phi_{S}\right)\right\rangle_{\mathrm{UT}}^{h}$ and $2\left\langle\sin \left(\phi_{S}\right)\right\rangle_{\mathrm{U} \perp}^{h}$ Fourier amplitudes differ only by the factor $\cos \theta_{\gamma^{*}} \simeq 1$ in the kinematic region of this measurement (cf. appendix A).

While disentangling all the different contributions to the $\sin \left(\phi_{S}\right)$ modulation will clearly require further detailed studies, the marked increase in magnitude of those modulations for charged pions and $K^{+}$with $z$ in the semi-exclusive region is especially intriguing. 

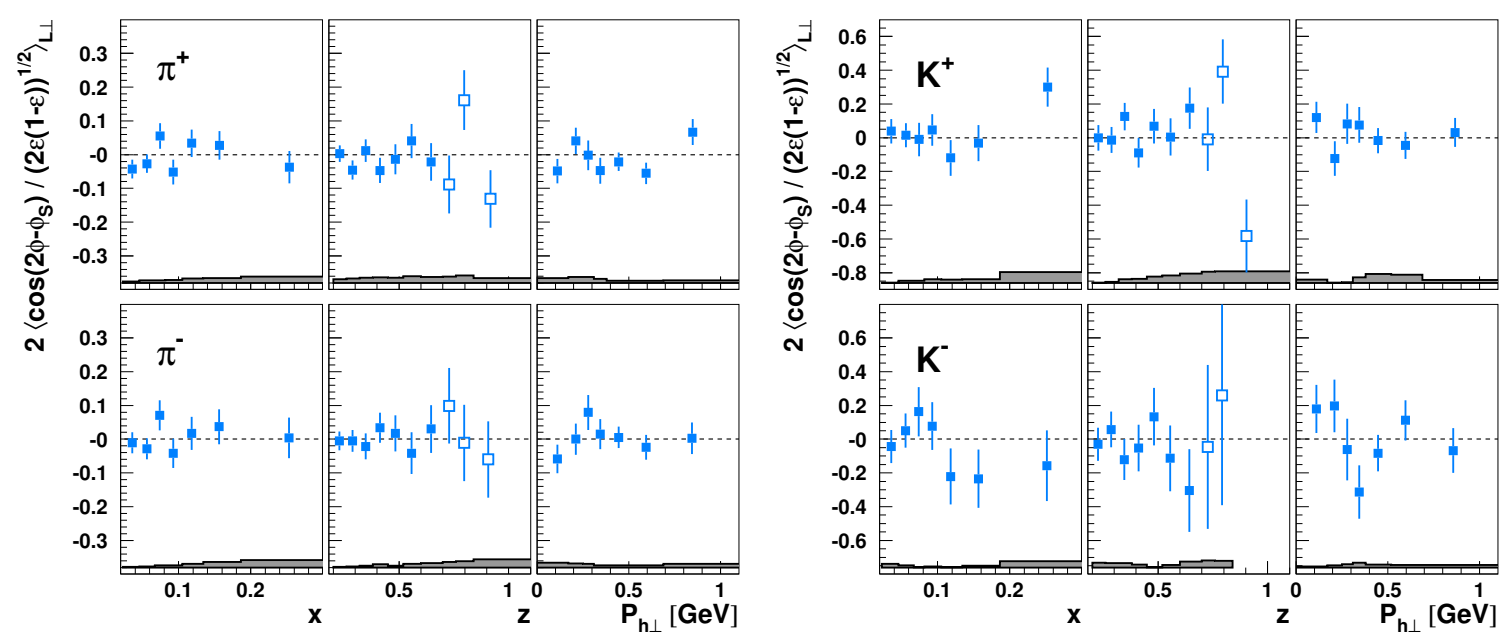

Figure 29. The $2\left\langle\cos \left(2 \phi-\phi_{S}\right) / \sqrt{2 \epsilon(1-\epsilon)}\right\rangle_{\mathrm{L} \perp}^{h}$ amplitudes for charged mesons (left: pions; right: kaons) presented either in bins of $x, z$, or $P_{h \perp}$. Data at large values of $z$, marked by open points in the $z$ projection, are not included in the other projections. Systematic uncertainties are given as bands, not including the additional scale uncertainty of $8.0 \%$ due to the precision of the targetpolarization determination.

In that respect, it appears worthwhile to point out that very sizable $\sin \left(\phi_{S}\right)$ modulations were observed in exclusive $\pi^{+}$electroproduction off transversely polarized protons [172].

The remaining two twist-3 Fourier amplitudes, the $\cos \left(2 \phi-\phi_{S}\right)$ and $\cos \left(\phi_{S}\right)$ modulations, require longitudinally polarized leptons in addition to transverse target polarization. As such, their statistical precision suffers from the relatively small lepton-beam polarization in these data. Again, several (and partially similar) terms contribute to those Fourier amplitudes as can be seen from eqs. (2.15) and (2.16), making a priori the interpretation in terms of specific TMDs difficult. Also in this case, Wandzura-Wilczek-type approximations might help to focus on only a few of the terms.

The $2\left\langle\cos \left(2 \phi-\phi_{S}\right) / \sqrt{2 \epsilon(1-\epsilon)}\right\rangle_{\mathrm{L} \perp}^{h}$ Fourier amplitudes for pions, charged kaons, and (anti)protons are presented in figures 29 and 30. None of those are found to be significantly different from zero. This is consistent with expectations [64] using Wandzura-Wilczek-type approximations of sub-percent level asymmetries. In such an approximation, only a term proportional to the worm-gear (II) $g_{1 \mathrm{~T}}^{q}$ and the ordinary $D_{1}^{q \rightarrow h}$ fragmentation function survives.

As in the case of the $2\left\langle\sin \left(\phi_{S}\right)\right\rangle_{\mathrm{U} \perp}^{h}$ Fourier amplitude, there is no contribution to the $2\left\langle\cos \left(2 \phi-\phi_{S}\right)\right\rangle_{\mathrm{L} \perp}^{h}$ Fourier amplitude from the longitudinal target-polarization component. Therefore, the $2\left\langle\cos \left(2 \phi-\phi_{S}\right)\right\rangle_{\mathrm{L} \perp}^{h}$ and $2\left\langle\cos \left(2 \phi-\phi_{S}\right)\right\rangle_{\mathrm{LT}}^{h}$ Fourier amplitudes differ only by the factor $\cos \theta_{\gamma^{*}} \simeq 1$ in the kinematic region of this measurement (cf. appendix $\mathrm{A}$ ).

Finally, the subleading-twist $2\left\langle\cos \left(\phi_{S}\right) / \sqrt{2 \epsilon(1-\epsilon)}\right\rangle_{\mathrm{L} \perp}^{h}$ Fourier amplitudes are depicted in figures 31 and 32. They are mostly consistent with zero, except for $K^{-}$, whose Fourier amplitudes are found to be incompatible with the NULL hypothesis at $95 \%$ confidence level.

In the Wandzura-Wilczek-type approximation, only the term proportional to $g_{\mathrm{T}}^{q}$ times $D_{1}^{q \rightarrow h}$ survives. The former quantifies the quark-flavor contribution to the inclusive-DIS 

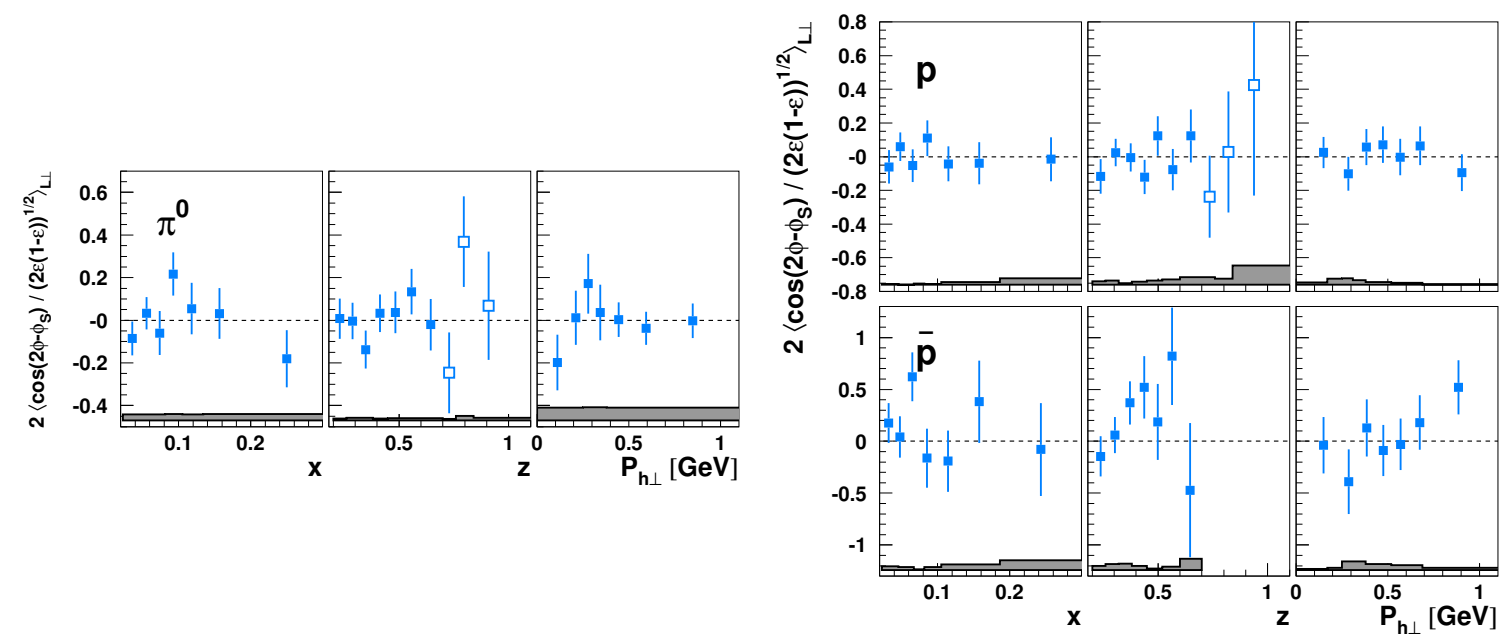

Figure 30. The $2\left\langle\cos \left(2 \phi-\phi_{S}\right) / \sqrt{2 \epsilon(1-\epsilon)}\right\rangle_{\mathrm{L} \perp}^{h}$ amplitudes for $\pi^{0}$ (left), protons, and antiprotons (right) presented either in bins of $x, z$, or $P_{h \perp}$. Data at large values of $z$, marked by open points in the $z$ projection, are not included in the other projections (no such high- $z$ points are available for antiprotons due to a lack of precision). Systematic uncertainties are given as bands, not including the additional scale uncertainty of $8.0 \%$ due to the precision of the target-polarization determination.

structure function $g_{2}$ via eq. (2.18). In this approximation, a small negative $2\left\langle\cos \left(\phi_{S}\right)\right\rangle_{\mathrm{LT}}^{h}$ Fourier amplitude of the order of $1-2 \%$ is predicted. While not necessarily favored by the data, such small negative asymmetries are not excluded in view of the overall precision of the data.

Without resorting to a Wandzura-Wilczek-type approximation, one can still reduce the number of contributing terms to $2\left\langle\cos \left(\phi_{S}\right)\right\rangle_{\mathrm{LT}}^{h}$ by looking at the $\cos \left(\phi_{S}\right)$ modulation integrated over transverse momentum because - like in the case of the $\sin \left(\phi_{S}\right)$ modulation - the $2\left\langle\cos \left(\phi_{S}\right)\right\rangle_{\mathrm{LT}}^{h}$ Fourier amplitude is not required to vanish upon integration over transverse hadron momentum. But in contrast to the $2\left\langle\sin \left(\phi_{S}\right)\right\rangle_{\mathrm{UT}}^{h}$ Fourier amplitude, two terms survive: the one discussed above involving $g_{\mathrm{T}}^{q}$ and the product of transversity and the twist-3 collinear $\widetilde{E}^{q \rightarrow h}(z)$ [82], as can be seen from eq. (2.17). This allows for a collinear extraction of transversity, at least in principle as the contribution of the $g_{\mathrm{T}}^{q}$ term needs to be subtracted. Furthermore, there exist similar considerations as for the $\sin \left(\phi_{S}\right)$ modulation, namely the usually incomplete integration over $\mathbf{P}_{h \perp}$ due to limitations in the geometric acceptance or kinematic requirements in experiments and the presently rather limited knowledge of the twist-3 fragmentation function $\widetilde{E}^{q \rightarrow h}(z)$.

Unlike the case of $2\left\langle\sin \left(\phi_{S}\right)\right\rangle_{\mathrm{U} \perp}^{h}$ and $2\left\langle\cos \left(2 \phi-\phi_{S}\right)\right\rangle_{\mathrm{L} \perp}^{h}$, in the experimental measurement of $2\left\langle\cos \left(\phi_{S}\right)\right\rangle_{\mathrm{L} \perp}$ amplitudes, relatively large contributions from the longitudinal target-polarization component can be expected due to the mixing discussed in appendix A. The double-spin asymmetry associated with the longitudinal polarization component is the typically sizable $A_{\|}^{h}$ related to the $A_{1}^{h}$ helicity asymmetry. It reaches values of 0.5 and higher $[162,175]$, and thus values that are in general much larger than those measured for azimuthal asymmetries. While suppressed because of the small value of $\theta_{\gamma^{*}}$, this contri- 

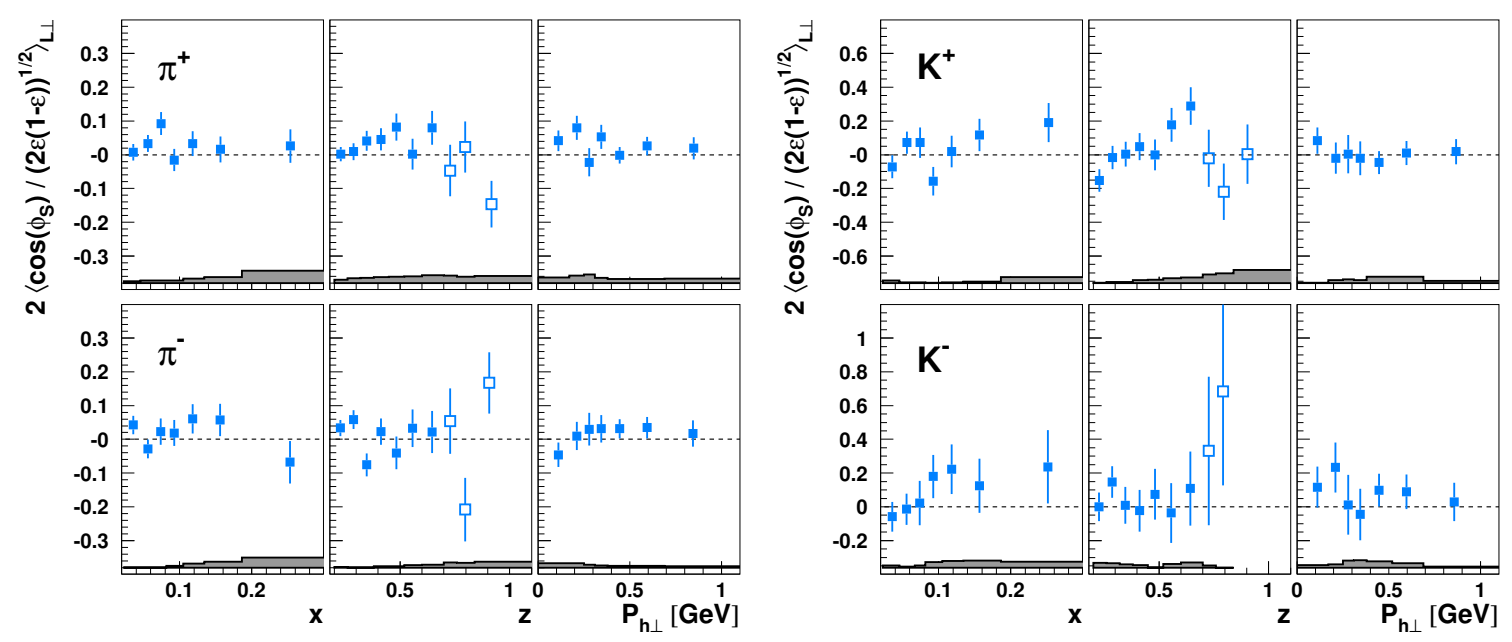

Figure 31. The $2\left\langle\cos \left(\phi_{S}\right) / \sqrt{2 \epsilon(1-\epsilon)}\right\rangle_{\mathrm{L} \perp}^{h}$ amplitudes for charged mesons (left: pions; right: kaons) presented either in bins of $x, z$, or $P_{h \perp}$. Data at large values of $z$, marked by open points in the $z$ projection, are not included in the other projections. Systematic uncertainties are given as bands, not including the additional scale uncertainty of $8.0 \%$ due to the precision of the target-polarization determination.
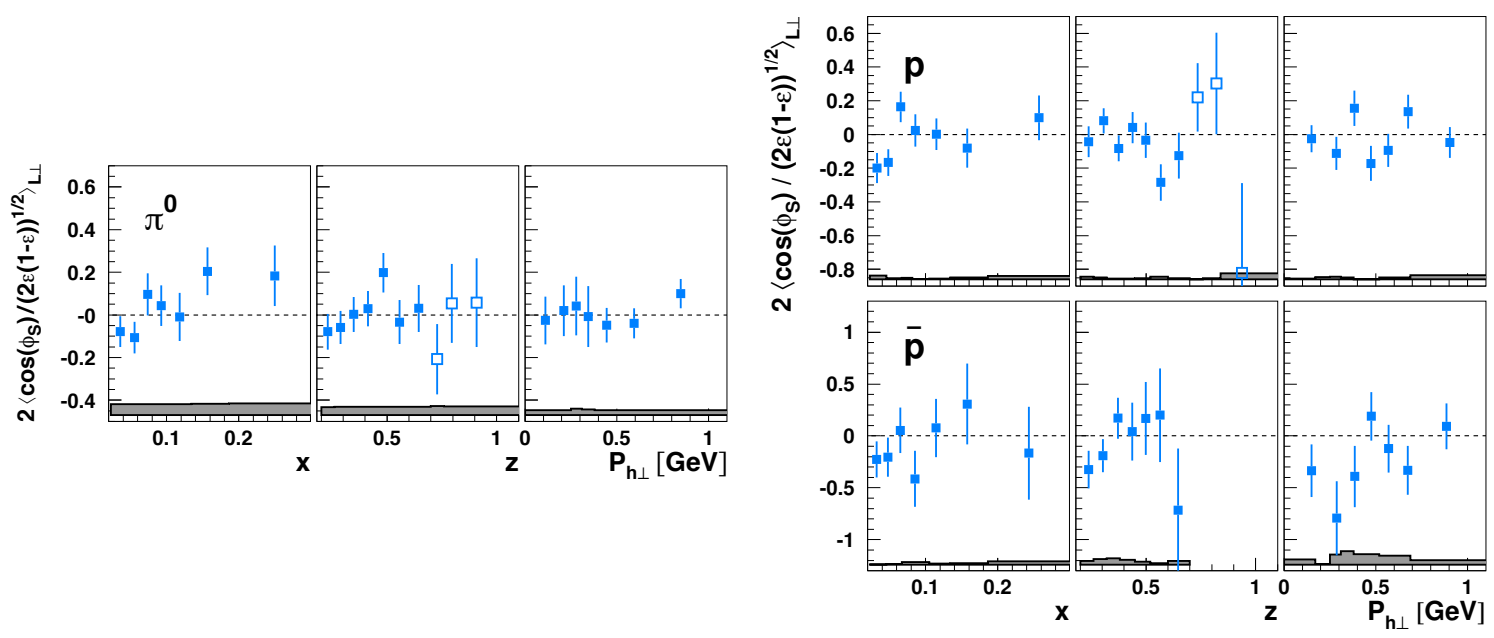

Figure 32. The $2\left\langle\cos \left(\phi_{S}\right) / \sqrt{2 \epsilon(1-\epsilon)}\right\rangle_{\mathrm{L} \perp}^{h}$ amplitudes for $\pi^{0}$ (left), protons, and antiprotons (right) presented either in bins of $x, z$, or $P_{h \perp}$. Data at large values of $z$, marked by open points in the $z$ projection, are not included in the other projections (no such high- $z$ points are available for antiprotons due to a lack of precision). Systematic uncertainties are given as bands, not including the additional scale uncertainty of $8.0 \%$ due to the precision of the target-polarization determination. 

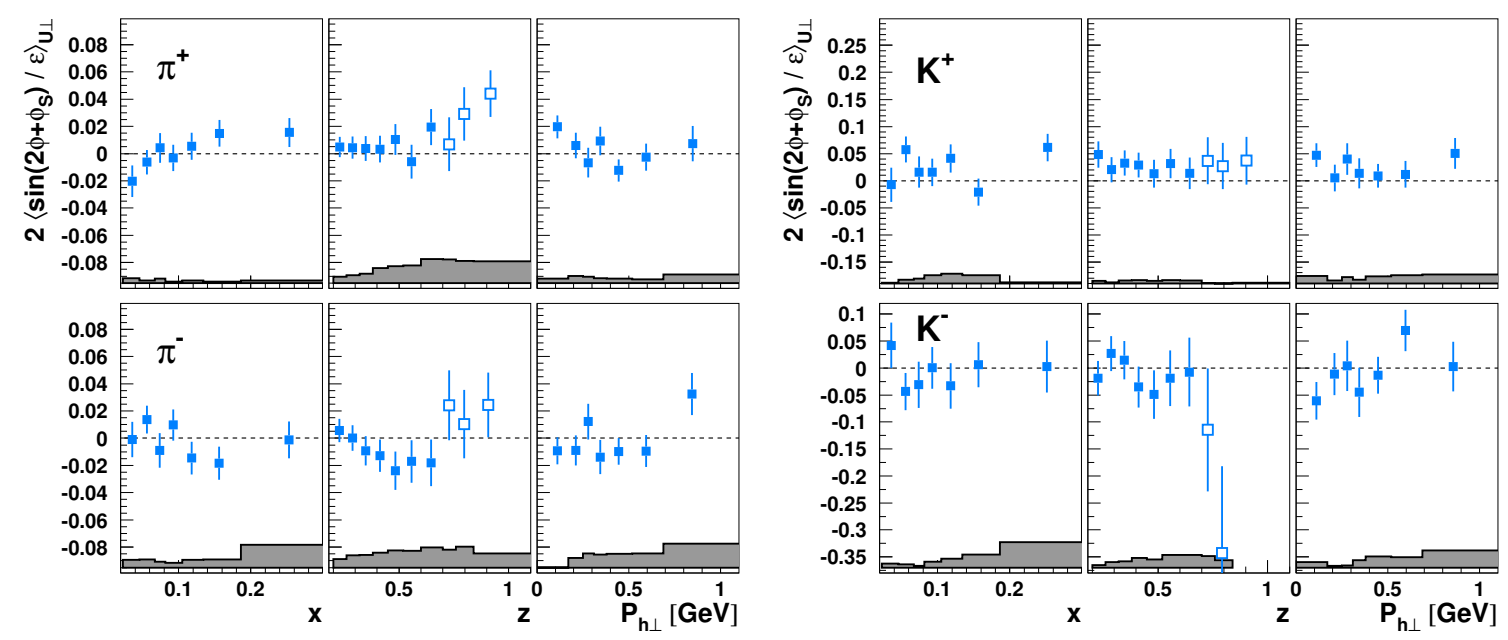

Figure 33. The $2\left\langle\sin \left(2 \phi+\phi_{S}\right) / \epsilon\right\rangle_{\mathrm{U} \perp}^{h}$ amplitudes for charged mesons (left: pions; right: kaons) presented either in bins of $x, z$, or $P_{h \perp}$. Data at large values of $z$, marked by open points in the $z$ projection, are not included in the other projections. Systematic uncertainties are given as bands, not including the additional scale uncertainty of $7.3 \%$ due to the precision of the target-polarization determination.

bution could still be substantial in comparison to the subleading-twist contributions from eq. (2.16) (cf. discussion in appendix A).

\subsection{Fourier moments arising solely from the longitudinal component of the target polarization}

In total ten Fourier components dependent on the transverse target polarization are extracted here. Of those, two arise solely because of a small longitudinal component of the proton polarization along the virtual-photon direction (cf. appendix A). They are the $2\left\langle\sin \left(2 \phi+\phi_{S}\right)\right\rangle_{\mathrm{U} \perp}^{h}$ Fourier amplitude of the transverse SSA and the $2\left\langle\cos \left(\phi+\phi_{S}\right)\right\rangle_{\mathrm{L} \perp}^{h}$ Fourier amplitude of the DSA, which are related to the $2\langle\sin (2 \phi)\rangle_{\mathrm{UL}}^{h}$ Fourier amplitude of the longitudinal SSA and the $2\langle\cos (\phi)\rangle_{\mathrm{LL}}^{h}$ Fourier amplitude of the longitudinal DSA, respectively. While $2\langle\sin (2 \phi)\rangle_{\mathrm{UL}}^{h}$ receives contributions at leading twist, $2\langle\cos (\phi)\rangle_{\mathrm{LL}}^{h}$ is of subleading twist.

The $2\langle\sin (2 \phi)\rangle_{\mathrm{UL}}^{h}$ Fourier amplitude provides access to the chiral-odd worm-gear (I) distribution $h_{1 \mathrm{~L}}^{\perp, q}\left(x, \mathbf{p}_{T}^{2}\right)$, which describes the distribution of transversely polarized quarks in a longitudinally polarized nucleon. As the final state involves unpolarized hadrons only, this chiral-odd TMD must couple to the chiral-odd Collins fragmentation function.

Vanishing $2\langle\sin (2 \phi)\rangle_{\mathrm{UL}}^{h}$ amplitudes for pions have been reported by the HERMES Collaboration in an analysis of single-spin asymmetries using longitudinally polarized hydrogen $[67,68]$ and deuterium [69] targets. The latter included also a measurement for $K^{+}$ mesons, which was found to be consistent with zero as well. The only non-vanishing signal so far has been reported by the CLAS Collaboration using a longitudinally polarized ammonia $\left({ }^{15} \mathrm{NH}_{3}\right)$ target (providing longitudinally polarized protons) [171]. The $2\langle\sin (2 \phi)\rangle_{\mathrm{UL}}^{\pi}$ Fourier amplitudes for charged pions are negative and of the order of $5 \%$ in magnitude. 

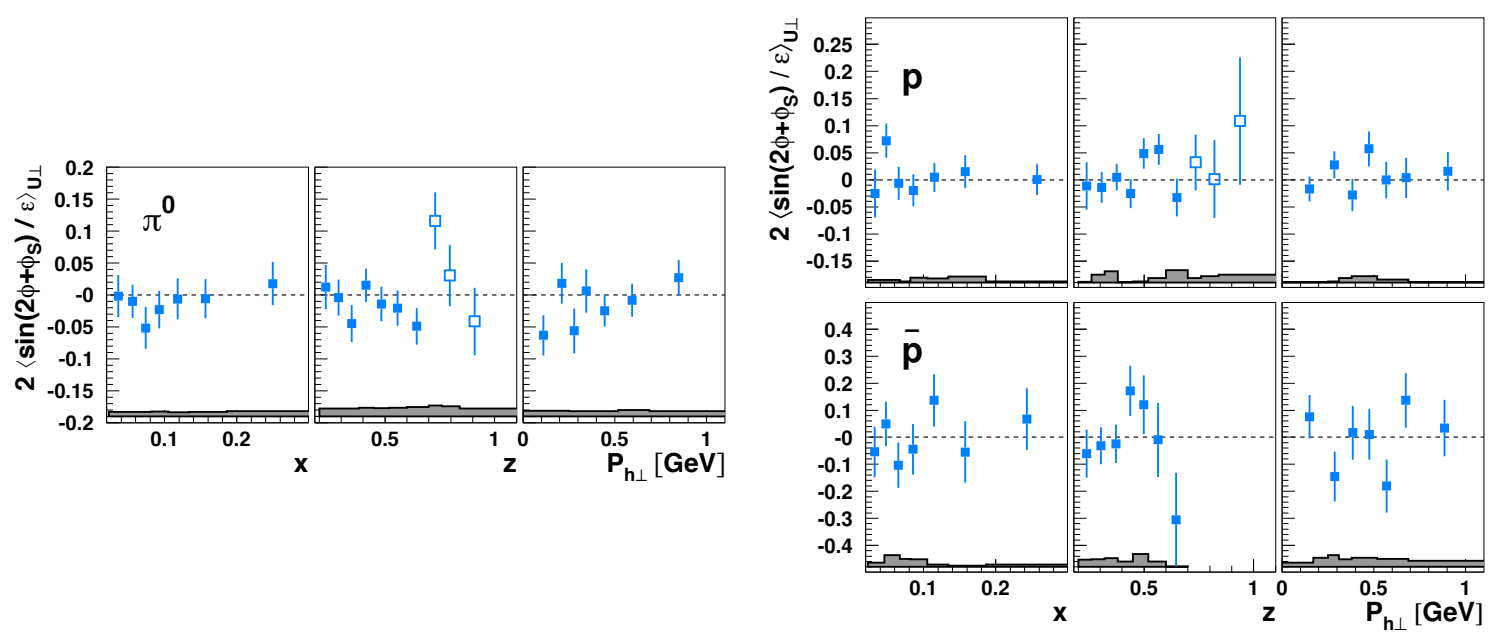

Figure 34. The $2\left\langle\sin \left(2 \phi+\phi_{S}\right) / \epsilon\right\rangle_{\mathrm{U} \perp}^{h}$ amplitudes for $\pi^{0}$ (left), protons, and antiprotons (right) presented either in bins of $x, z$, or $P_{h \perp}$. Data at large values of $z$, marked by open points in the $z$ projection, are not included in the other projections (no such high- $z$ points are available for antiprotons due to a lack of precision). Systematic uncertainties are given as bands, not including the additional scale uncertainty of $7.3 \%$ due to the precision of the target-polarization determination.

The $2\left\langle\sin \left(2 \phi+\phi_{S}\right)\right\rangle_{\mathrm{U} \perp}^{h}$ amplitudes in the Fourier decomposition of the experimentally measured cross section, which are not sensitive to a structure function of the transverse target spin-dependent cross-section contribution $\sigma_{\mathrm{UT}}^{h}$ in eq. (2.1), are related to the $2\langle\sin (2 \phi)\rangle_{\mathrm{UL}}^{h}$ amplitudes through

$$
2\left\langle\sin \left(2 \phi+\phi_{S}\right)\right\rangle_{\mathrm{U} \perp}^{h} \simeq \frac{1}{2} \sin \theta_{\gamma^{*}} 2\langle\sin (2 \phi)\rangle_{\mathrm{UL}}^{h}
$$

(cf. appendix A). ${ }^{24}$ Therefore, a potential signal for $h_{1 \mathrm{~L}}^{\perp, q}\left(x, \mathbf{p}_{T}^{2}\right)$ is additionally suppressed by at least an order of magnitude compared to corresponding measurements using longitudinally polarized targets.

The $2\left\langle\sin \left(2 \phi+\phi_{S}\right) / \epsilon\right\rangle_{\mathrm{U} \perp}^{h}$ Fourier amplitudes for pions, charged kaons, as well as for (anti)protons extracted in the presented analysis are shown in figures 33 and 34 . They are primarily consistent with zero and thus in agreement with the previous $2\langle\sin (2 \phi)\rangle_{\mathrm{UL}}^{h}$ related measurements, where data are available. There is a tendency for a non-vanishing signal for positive pions at very large $z$, e.g., when approaching the exclusive region, similar to what has been discussed for the $2\left\langle\sin \left(2 \phi-\phi_{S}\right)\right\rangle_{\mathrm{U} \perp}^{h}$ Fourier amplitude in section 4.5. An analogous Fourier decomposition of the transverse SSA in exclusive $\pi^{+}$electroproduction [172] does result in $\sin \left(2 \phi+\phi_{S}\right)$ modulations not dissimilar to the behavior observed here in the large- $z$ region, hinting at a non-vanishing $2\langle\sin (2 \phi)\rangle_{\mathrm{UL}}^{\pi^{+}}$Fourier amplitude in the exclusive regime and possibly in the semi-exclusive region probed here. On the other hand, the direct measurement of the $2\langle\sin (2 \phi)\rangle_{\mathrm{U} \|}^{\pi^{+}}$Fourier amplitude in exclusive $\pi^{+}$electropro-

\footnotetext{
${ }^{24}$ Through the same longitudinal target-polarization component, $h_{1 \mathrm{~L}}^{\perp, q}\left(x, \mathbf{p}_{T}^{2}\right)$ contributes with equal magnitude also to $2\left\langle\sin \left(2 \phi-\phi_{S}\right)\right\rangle_{\mathrm{U} \perp}^{h}$ as discussed before. However, in that case it has to compete with the genuine transverse-polarization effects introduced in section 2.2.5.
} 
duction gives only $0.05 \pm 0.05$ [176], likely too small to produce a sizable $2\left\langle\sin \left(2 \phi+\phi_{S}\right)\right\rangle_{\mathrm{U} \perp}^{\pi^{+}}$ Fourier amplitude through the mixing of longitudinal and transverse target-polarization components. ${ }^{25}$

The $K^{+} 2\left\langle\sin \left(2 \phi+\phi_{S}\right) / \epsilon\right\rangle_{\mathrm{U} \perp}$ Fourier amplitude presented here might be the notable exception as - somewhat unexpectedly - it is positive over essentially the entire $z$ range. It is incompatible with the NULL hypothesis at $95 \%$ confidence as already presented in table 9 . This points to a possibly sizable $2\langle\sin (2 \phi)\rangle_{\mathrm{UL}}^{K^{+}}$asymmetry and thus indirectly to a sizable worm-gear (I) $h_{1 \mathrm{~L}}^{\perp, q}\left(x, \mathbf{p}_{T}^{2}\right)$. In particular, taking into account the factor $\frac{1}{2} \sin \theta_{\gamma^{*}}$ that relates the two Fourier amplitudes and that amounts on average to 0.04 (cf. eq. (4.2) and appendix A), $2\langle\sin (2 \phi)\rangle_{\mathrm{UL}}^{K^{+}}$Fourier amplitudes of the order $30 \%$ can be expected. No measurement of $2\langle\sin (2 \phi)\rangle_{\mathrm{UL}}^{K^{+}}$for a proton target is presently available. A direct comparison of $2\langle\sin (2 \phi)\rangle_{\mathrm{UL}}^{K^{+}}$to the $2\left\langle\sin \left(2 \phi+\phi_{S}\right)\right\rangle_{\mathrm{U} \perp}^{K^{+}}$presented here is thus not possible. Results for the $2\langle\sin (2 \phi)\rangle_{\mathrm{U} \|}^{K^{+}}$Fourier amplitude for a deuteron target are consistent with zero and within the achieved precision incompatible with magnitudes of tens of percent. On the other hand, there have been various instances where the $K^{+}$result exceeds significantly the magnitudes for pions, prominent cases being the Sivers as well as the Collins asymmetries presented here. Recalling that $h_{1 \mathrm{~L}}^{\perp, q}\left(x, \mathbf{p}_{T}^{2}\right)$ couples to the Collins fragmentation function in $2\langle\sin (2 \phi)\rangle_{\mathrm{UL}}^{h}$ and that for $\pi^{+}$there are significant cancelation effects due to the opposite signs for the favored and disfavored Collins fragmentation functions of pions, larger $K^{+}$modulations can be expected if, for example, only $H_{1}^{\perp, u \rightarrow K^{+}}$is sizable as found in ref. [140].

The $2\left\langle\cos \left(\phi+\phi_{S}\right) / \sqrt{2 \epsilon(1-\epsilon)}\right\rangle_{\mathrm{L} \perp}^{h}$ Fourier amplitudes for pions, for charged kaons, as well as for (anti-)protons extracted in this analysis are shown in figures 35 and 36 . They arise through the small longitudinal target-polarization component from the subleadingtwist $\cos (\phi)$ azimuthal modulation of the longitudinal DSA. The latter may arise through the "polarized Cahn effect" [105, 177, 178], which combines transverse momenta of longitudinally polarized quarks in a longitudinally polarized nucleon with the transverse momentum acquired in the fragmentation process and as such is sensitive to the transversemomentum dependence of the helicity distribution, $g_{1}\left(x, \mathbf{p}_{T}^{2}\right)$. No significant signal for such modulation has been reported so far, neither for unidentified hadrons from a longitudinally polarized ${ }^{6} \mathrm{LiD}$ (effectively a deuteron) target at the COMPASS experiment [179] nor for identified pions (and kaons) from a longitudinally polarized hydrogen (deuterium) target by the HERMES Collaboration [175]. This is consistent with the vanishing signal for $2\left\langle\cos \left(\phi+\phi_{S}\right)\right\rangle_{\mathrm{L} \perp}^{h}$ reported here.

\footnotetext{
${ }^{25} \mathrm{~A}$ conclusive evaluation is hampered by the precision of the data and the possibility that the $2\langle\sin (2 \phi)\rangle_{\mathrm{U} \|}^{\pi^{+}}$result for exclusive $\pi^{+}$electo-production received in turn contributions from a $2\left\langle\sin \left(2 \phi-\phi_{S}\right)\right\rangle_{\mathrm{UT}}^{\pi^{+}}$term in exclusive $\pi^{+}$electroproduction, requiring a combined analysis of all three modulations, $2\langle\sin (2 \phi)\rangle_{\mathrm{U} \|}^{\pi^{+}}, 2\left\langle\sin \left(2 \phi-\phi_{S}\right)\right\rangle_{\mathrm{U} \perp}^{\pi^{+}}$, and $2\left\langle\sin \left(2 \phi+\phi_{S}\right)\right\rangle_{\mathrm{U} \perp}^{\pi^{+}}$, along the lines of what was done in ref. [70].
} 

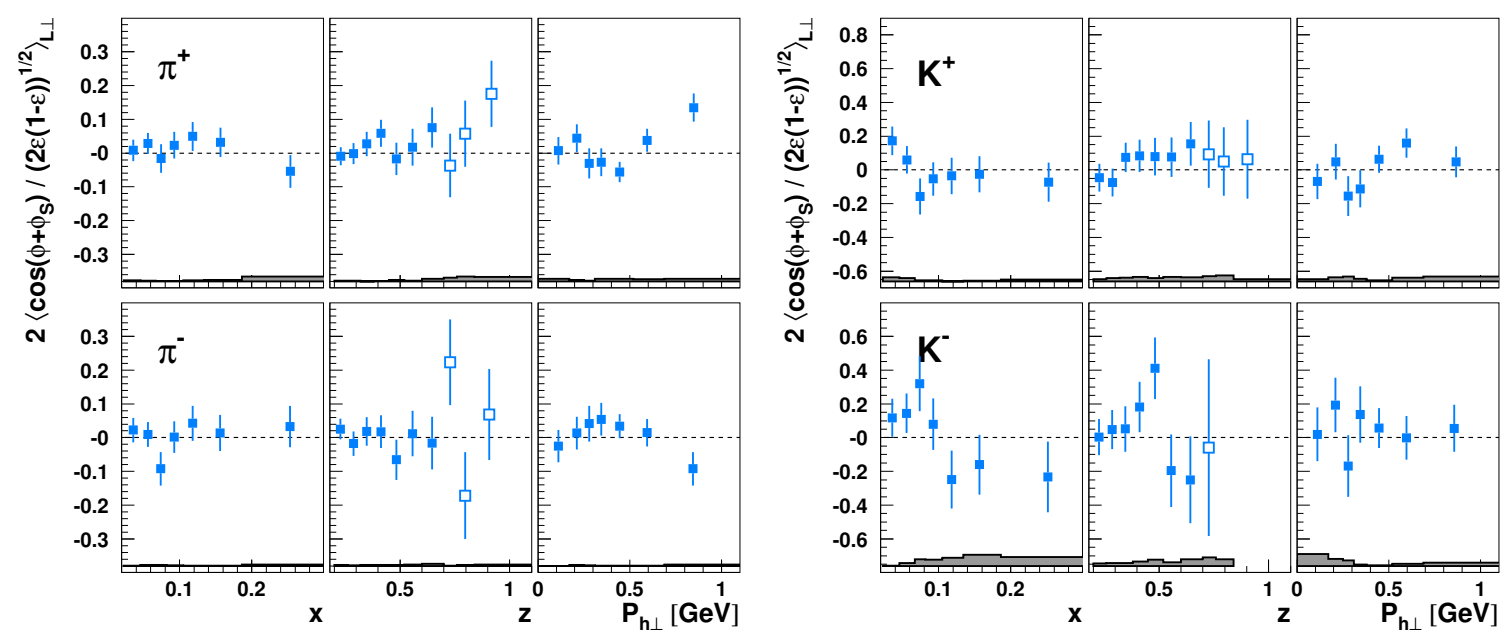

Figure 35. The $2\left\langle\cos \left(\phi+\phi_{S}\right) / \sqrt{2 \epsilon(1-\epsilon)}\right\rangle_{\mathrm{L} \perp}^{h}$ amplitudes for charged mesons (left: pions; right: kaons) presented either in bins of $x, z$, or $P_{h \perp}$. Data at large values of $z$, marked by open points in the $z$ projection, are not included in the other projections. Systematic uncertainties are given as bands, not including the additional scale uncertainty of $8.0 \%$ due to the precision of the targetpolarization determination.
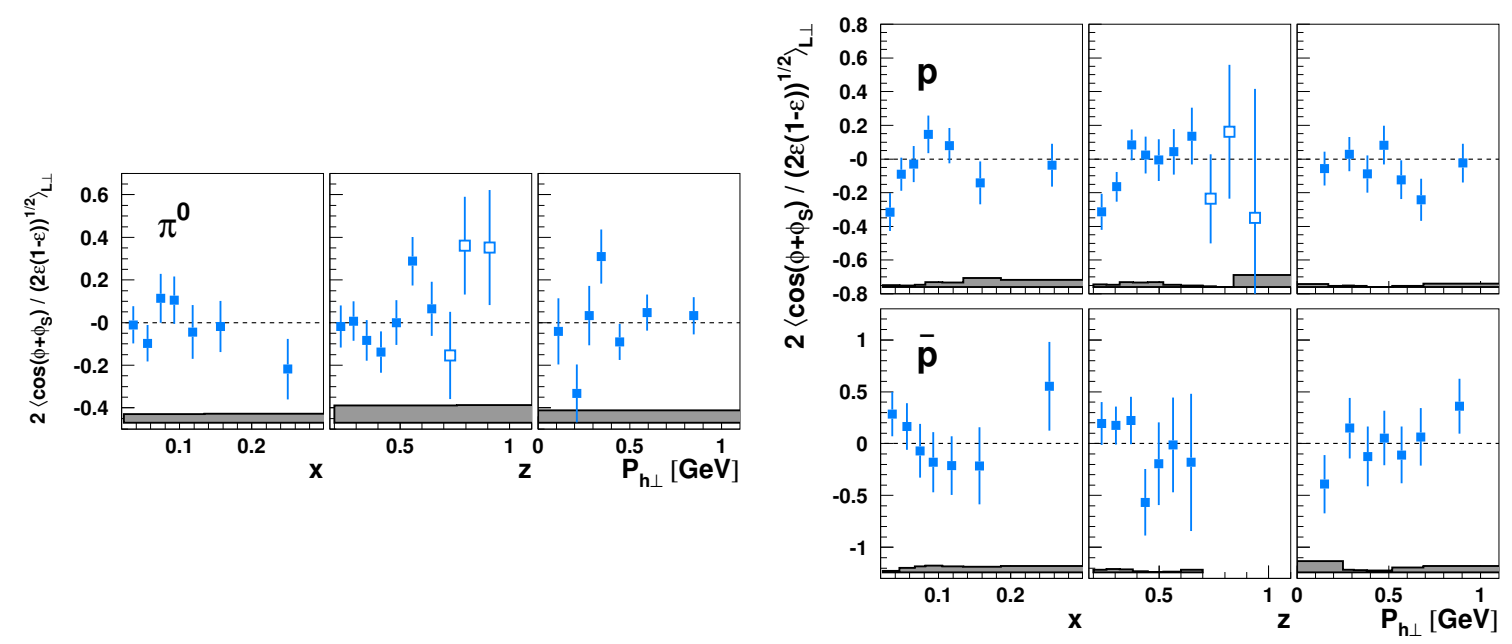

Figure 36. The $2\left\langle\cos \left(\phi+\phi_{S}\right) / \sqrt{2 \epsilon(1-\epsilon)}\right\rangle_{\mathrm{L} \perp}^{h}$ amplitudes for $\pi^{0}$ (left), protons, and antiprotons (right) presented either in bins of $x, z$, or $P_{h \perp}$. Data at large values of $z$, marked by open points in the $z$ projection, are not included in the other projections (no such high- $z$ points are available for antiprotons due to a lack of precision). Systematic uncertainties are given as bands, not including the additional scale uncertainty of $8.0 \%$ due to the precision of the target-polarization determination. 


\section{Conclusion}

A comprehensive discussion of azimuthal single- and double-spin asymmetries in semiinclusive leptoproduction of pions, charged kaons, protons, and antiprotons from transversely polarized protons at HERMES has been presented. These asymmetries include the previously published HERMES results on Collins and Sivers asymmetries [29, 40], which have been extended to include protons and antiprotons and also to an extraction in a three-dimensional binning in $x, z$, and $P_{h \perp}$. In addition, the large- $z$ region of $z>0.7$ is explored to study the transition from the semi-inclusive to the exclusive regime.

Furthermore, the set of azimuthal asymmetries measured include those arising from the leading-twist pretzelosity and worm-gear (II) distributions, four subleading-twist modulations, and two modulations that contribute to the $e^{ \pm} p$ cross-section through the small but non-vanishing longitudinal target-polarization component in experiments where the target is polarized perpendicular to the beam direction. No sign of a non-zero pretzelosity is found, while the non-vanishing $\cos \left(\phi-\phi_{S}\right)$ modulations for pions provide evidence for a sizable worm-gear (II) distribution, $g_{1 \mathrm{~T}}^{q}\left(x, \mathbf{p}_{T}^{2}\right)$. The subleading-twist contributions and the ones from the longitudinal target-spin component are mostly consistent with zero. A rather notable exception are the large $\sin \left(\phi_{S}\right)$ modulations for charged pions and $K^{+}$.

All modulations were studied as functions of $x, z$, and $P_{h \perp}$ individually as well as simultaneously binned in all the three kinematic variables, except for $\pi^{0}$ and antiprotons, in which case the corresponding yields were not sufficient to allow such three-dimensional binning. Fourier amplitudes were extracted including or excluding the kinematic prefactor arising from the photon spin-density matrix accompanying each specific cross-section contribution. This allows for a simpler comparison with other experiments or theoretical calculations as experiment-specific integration over kinematic variables is minimized. The results for the azimuthal modulations are supplemented by information on the unpolarized cross section, in particular, distributions in rapidity as well as of transverse momentum vs. the hard scale $Q^{2}$. Those are expected to facilitate the interpretation of the modulation in global analyses within the TMD framework.

\section{Acknowledgments}

This paper is dedicated to our recently deceased colleagues Robert Avakian, Stanislav Belostotski, Harold E. Jackson, and Bohdan Marianski.

This work would not have been possible without the continuous input by our theory colleagues; especially appreciated is the input by M. Boglione for providing calculations of the Sivers asymmetry and by M. Diehl in many very valuable discussions. We gratefully acknowledge the DESY management for its support and the staff at DESY, in particular, the data-preservation group, as well as the collaborating institutions for their significant effort. This work was supported by the State Committee of Science of the Republic of Armenia, Grant No. 18T-1C180; the FWO-Flanders and IWT, Belgium; the Natural Sciences and Engineering Research Council of Canada; the National Natural Science Foundation of China; the Alexander von Humboldt Stiftung, the German Bundesministerium für Bildung 


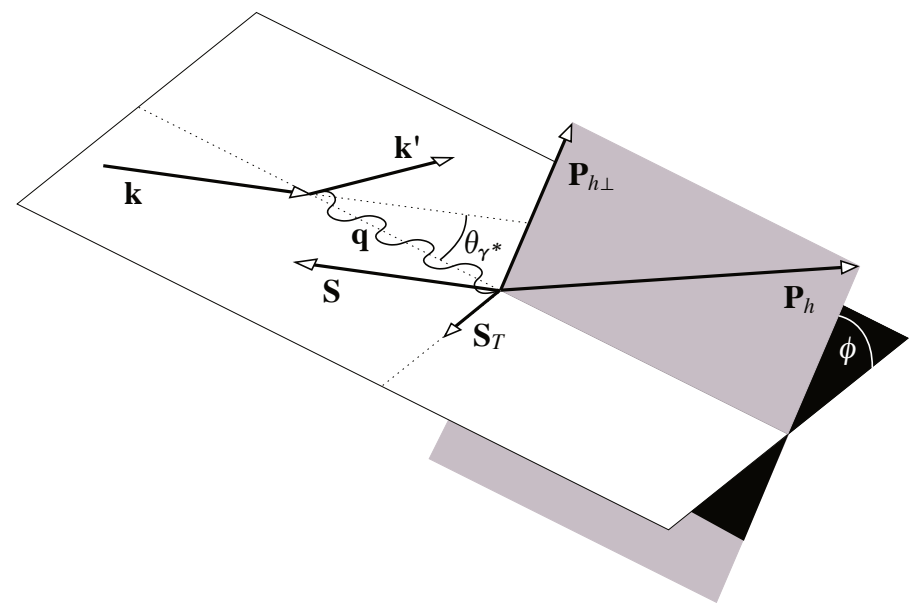

Figure 37. Illustration of the mixing of longitudinal and transverse target polarizations w.r.t. the virtual-photon direction when polarizing the target along the lepton-beam direction. In this particular case of longitudinal target polarization, a small component transverse to the virtual-photon direction arises that is proportional to $\sin \theta_{\gamma^{*}}$.

und Forschung (BMBF), and the Deutsche Forschungsgemeinschaft (DFG); the Italian Istituto Nazionale di Fisica Nucleare (INFN); the MEXT, JSPS, and G-COE of Japan; the Dutch Foundation for Fundamenteel Onderzoek der Materie (FOM); the Russian Academy of Science and the Russian Federal Agency for Science and Innovations; the Basque Foundation for Science (IKERBASQUE), Spain; the U.K. Engineering and Physical Sciences Research Council, the Science and Technology Facilities Council, and the Scottish Universities Physics Alliance; as well as the U.S. Department of Energy (DOE) and the National Science Foundation (NSF).

\section{A Contribution from longitudinal target polarization}

The interest in leptoproduction data on transversely polarized protons lies in the various semi-inclusive structure functions discussed in section 2.2. As the target-polarization direction in an actual experiment refers to the lepton-beam direction for the reference axis and not to the virtual-photon direction used in theory, most of the azimuthal modulations measured here receive contributions from the resulting non-vanishing longitudinal component of the target polarization with respect to the virtual-photon direction (see figure 37). This leads to additional moments as compared to, e.g., eq. (2.1), resulting in [102]

$$
\begin{aligned}
\sigma^{h}\left(\phi, \phi_{S}\right)= & \sigma_{\mathrm{UU}}^{h}\left\{1+2\langle\cos (\phi)\rangle_{\mathrm{UU}}^{h} \cos (\phi)+2\langle\cos (2 \phi)\rangle_{\mathrm{UU}}^{h} \cos (2 \phi)\right. \\
+ & S_{L}\left[2\langle\sin (\phi)\rangle_{\mathrm{U} \|}^{h} \sin (\phi)+2\langle\sin (2 \phi)\rangle_{\mathrm{U} \|}^{h} \sin (2 \phi)+2\langle\sin (3 \phi)\rangle_{\mathrm{U} \|}^{h} \sin (3 \phi)\right. \\
& \left.+\lambda_{l}\left(2\langle\cos (0 \phi)\rangle_{\mathrm{L} \|}^{h} \cos (0 \phi)+2\langle\cos (\phi)\rangle_{\mathrm{L} \|}^{h} \cos (\phi)+2\langle\cos (2 \phi)\rangle_{\mathrm{L} \|}^{h} \cos (2 \phi)\right)\right] \\
+ & S_{T}\left[2\left\langle\sin \left(\phi-\phi_{S}\right)\right\rangle_{\mathrm{U} \perp}^{h} \sin \left(\phi-\phi_{S}\right)+2\left\langle\sin \left(\phi+\phi_{S}\right)\right\rangle_{\mathrm{U} \perp}^{h} \sin \left(\phi+\phi_{S}\right)\right. \\
& +2\left\langle\sin \left(3 \phi-\phi_{S}\right)\right\rangle_{\mathrm{U} \perp}^{h} \sin \left(3 \phi-\phi_{S}\right)+2\left\langle\sin \left(\phi_{S}\right)\right\rangle_{\mathrm{U} \perp}^{h} \sin \left(\phi_{S}\right)
\end{aligned}
$$




$$
\begin{gathered}
+2\left\langle\sin \left(2 \phi-\phi_{S}\right)\right\rangle_{\mathrm{U} \perp}^{h} \sin \left(2 \phi-\phi_{S}\right)+2\left\langle\sin \left(2 \phi+\phi_{S}\right)\right\rangle_{\mathrm{U} \perp}^{h} \sin \left(2 \phi+\phi_{S}\right) \\
+\lambda_{l}\left(2\left\langle\cos \left(\phi-\phi_{S}\right)\right\rangle_{\mathrm{L} \perp}^{h} \cos \left(\phi-\phi_{S}\right)+2\left\langle\cos \left(\phi+\phi_{S}\right)\right\rangle_{\mathrm{L} \perp}^{h} \cos \left(\phi+\phi_{S}\right)\right. \\
\left.\left.\left.+2\left\langle\cos \left(\phi_{S}\right)\right\rangle_{\mathrm{L} \perp}^{h} \cos \left(\phi_{S}\right)+2\left\langle\cos \left(2 \phi-\phi_{S}\right)\right\rangle_{\mathrm{L} \perp}^{h} \cos \left(2 \phi-\phi_{S}\right)\right)\right]\right\},
\end{gathered}
$$

where the cross section averaged over the polarization states and integrated over $\phi$ and $\phi_{S}$ is represented by $\sigma_{\mathrm{UU}}^{h}$ and has been factored out.

The size of the component of the nucleon-spin vector that is longitudinal to the virtualphoton direction depends on $\theta_{\gamma^{*}}$, the polar angle between the incoming-beam and the virtual-photon directions. Hence it strongly depends on the event kinematics. At HERMES kinematics, $\sin \theta_{\gamma^{*}}$ is of the order of 0.1 but can be as large as 0.2 for events at very large $x$. Here, $\sin \theta_{\gamma^{*}}$ is evaluated from the lepton kinematics as

$$
\sin \theta_{\gamma^{*}}=\frac{2 x M}{Q} \sqrt{\frac{1-y-y^{2} x^{2} M^{2} / Q^{2}}{1+4 x^{2} M^{2} / Q^{2}}} .
$$

Its average values are presented in figure 38 for $\pi^{+}$(similar for the other hadrons) in the same three-dimensional kinematic binning used also for the asymmetry measurement. Likewise, they are presented in figure 39 for the one-dimensional binning of mesons. The longitudinal polarization components are also provided as tabulated values in the supplementary material.

The contributions from the transverse and longitudinal components can only be disentangled using data from targets with both polarization orientations. Such analysis was presented in ref. [70] by the HERMES Collaboration, using both data for the Sivers and Collins type modulations for transverse target polarization [28] and the $\sin \phi$ modulation for longitudinal polarization [67]. It is based on the inversion of the mixing matrix

$$
\left(\begin{array}{c}
2\langle\sin (\phi)\rangle_{\mathrm{U} \|} \\
2\left\langle\sin \left(\phi-\phi_{S}\right)\right\rangle_{\mathrm{U} \perp} \\
2\left\langle\sin \left(\phi+\phi_{S}\right)\right\rangle_{\mathrm{U} \perp}
\end{array}\right)=\left(\begin{array}{ccc}
\cos \theta_{\gamma^{*}} & -\sin \theta_{\gamma^{*}} & -\sin \theta_{\gamma^{*}} \\
\frac{1}{2} \sin \theta_{\gamma^{*}} & \cos \theta_{\gamma^{*}} & 0 \\
\frac{1}{2} \sin \theta_{\gamma^{*}} & 0 & \cos \theta_{\gamma^{*}}
\end{array}\right)\left(\begin{array}{c}
2\langle\sin (\phi)\rangle_{\mathrm{UL}} \\
2\left\langle\sin \left(\phi-\phi_{S}\right)\right\rangle_{\mathrm{UT}} \\
2\left\langle\sin \left(\phi+\phi_{S}\right)\right\rangle_{\mathrm{UT}}
\end{array}\right),
$$

which is valid up to corrections of order $\sin ^{2} \theta_{\gamma^{*}}$ [102]. Similar expressions are obtained for the other modulations studied here by interchanging in eq. (A.3)

(i) $\sin \left(\phi \pm \phi_{S}\right) \leftrightarrow \sin \left(n \phi \pm \phi_{S}\right)$ and $\sin (\phi) \leftrightarrow \sin (n \phi)$ for $n>0$,

(ii) $\sin \leftrightarrow \cos$ in case of longitudinal beam polarization.

Note that some of the elements of the moments vectors might then be identical to zero (cf. eqs. (2.1) and (A.1)), e.g., $\langle\sin (3 \phi)\rangle_{\mathrm{LL}}$, at least in the one-photon-exchange approximation.

The $\phi$-independent SSA relates to its theory counterpart via

$$
2\left\langle\sin \left(\phi_{S}\right)\right\rangle_{\mathrm{U} \perp}=\cos \theta_{\gamma^{*}} 2\left\langle\sin \left(\phi_{S}\right)\right\rangle_{\mathrm{UT}},
$$




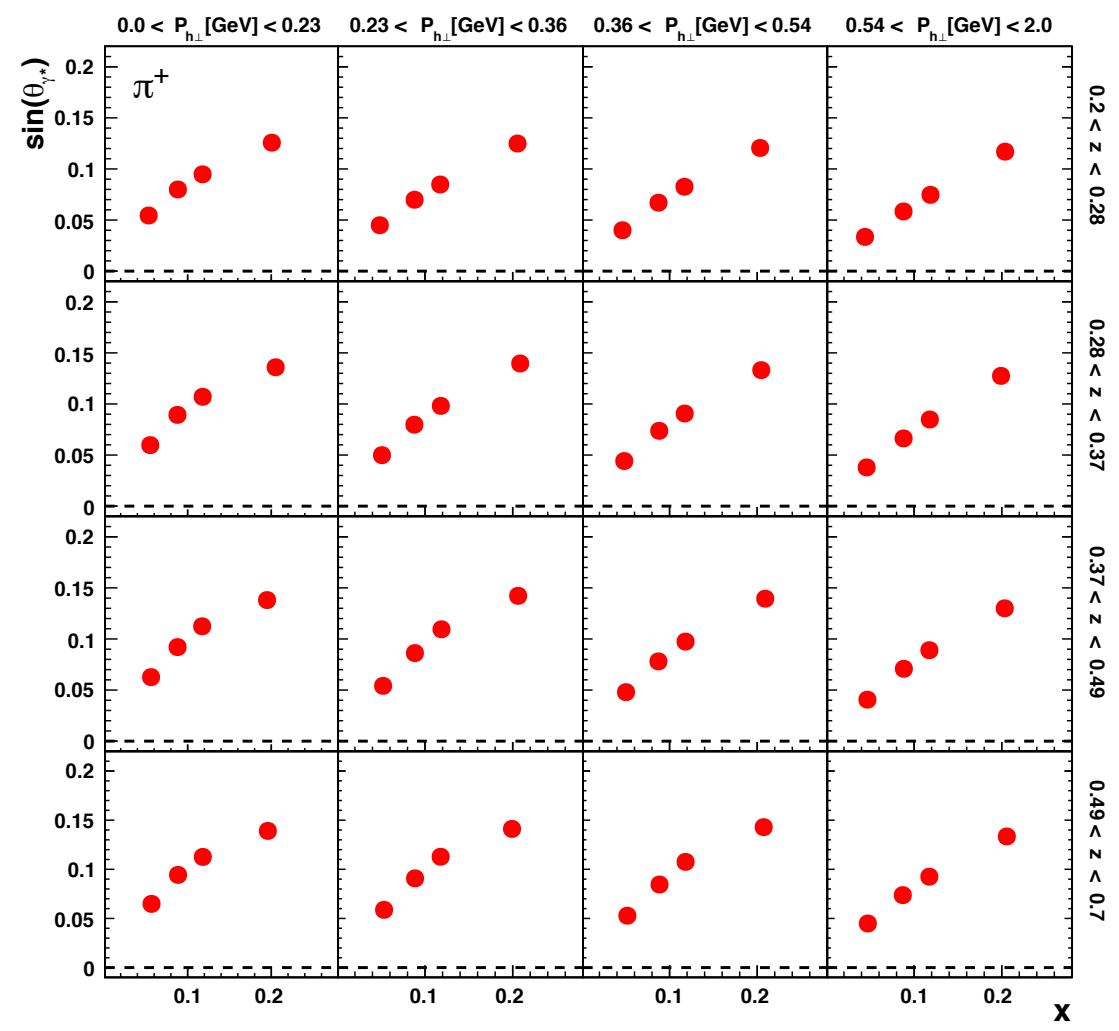

Figure 38. Average $\sin \theta_{\gamma^{*}}$ as a function of $x$ in bins of $z$ and $P_{h \perp}$ in the same three-dimensional binning used for the analysis of azimuthal asymmetries of charged mesons (using here $\pi^{+}$data as an example).

while the mixing of the $\phi$-independent double-spin asymmetries can be expressed as

$$
\left(\begin{array}{c}
\langle\cos 0\rangle_{\mathrm{L} \|} \\
2\left\langle\cos \left(\phi_{S}\right)\right\rangle_{\mathrm{L} \perp}
\end{array}\right)=\left(\begin{array}{cc}
\cos \theta_{\gamma^{*}} & -\sin \theta_{\gamma^{*}} \\
\sin \theta_{\gamma^{*}} & \cos \theta_{\gamma^{*}}
\end{array}\right)\left(\begin{array}{c}
\langle\cos 0\rangle_{\mathrm{LL}} \\
2\left\langle\cos \left(\phi_{S}\right)\right\rangle_{\mathrm{LT}}
\end{array}\right) .
$$

The experimental challenge consists in combining measurements using transversely and longitudinally polarized targets under similar conditions, which among others requires identical kinematic binning for the two data sets. For the analysis presented here, such a matching data set is missing. In particular, the use of a threshold Cherenkov counter during data taking with a longitudinally polarized hydrogen target prohibits the measurement of the relevant kaon and also (anti)proton asymmetries. Therefore, no attempt has been made to disentangle the structure functions related to transversely and longitudinally polarized protons. Future data and/or parameterization of the relevant longitudinal-spin asymmetries might be used instead to extract the purely transverse structure functions.

Nevertheless, while a precise quantitative evaluation of the effect for all the SSAs and DSAs of this measurement is currently out of reach, a few qualitative comments might be in order. In general, most azimuthal moments presented here and elsewhere for longitudinal target polarization are of similar order of magnitude, e.g., below 0.1 in magnitude. The 


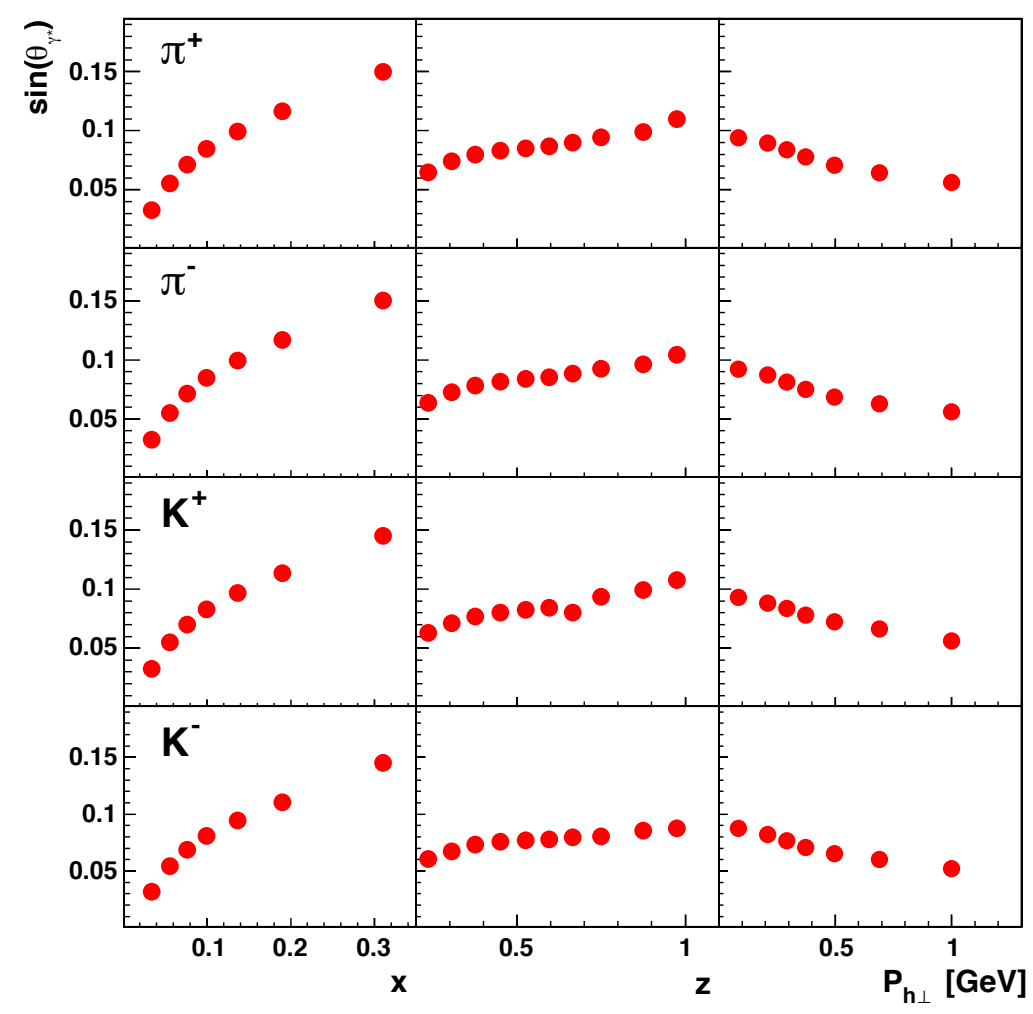

Figure 39. Average $\sin \theta_{\gamma^{*}}$ as a function of $x, z$, or $P_{h \perp}$ for charged mesons as labeled.

corrections are thus small as already noted for the Sivers and Collins asymmetries in ref. [70]. This is not quite the case though for the $\phi$-independent DSA, $2\left\langle\cos \left(\phi_{S}\right)\right\rangle_{\mathrm{L} \perp}$, which receives rather large contributions from the azimuthally uniform structure function arising from the quark-helicity distribution. Those are up to an order of magnitude larger [175] than the typical azimuthal moments and increase with $x$ as does the longitudinal targetpolarization component, which needs to be considered when interpreting the $2\left\langle\cos \left(\phi_{S}\right)\right\rangle_{\mathrm{L} \perp}$ results. As an example, the contribution from $A_{\|}$has been evaluated using the HERMES measurement [175] scaled by the corresponding average longitudinal target-spin component of each $x$ bin, shown in figure 40 .

There are notable exceptions to this general discussion. Three azimuthal asymmetries, namely the $2\left\langle\sin \left(\phi_{S}\right)\right\rangle_{\mathrm{U} \perp}$, the $2\left\langle\sin \left(3 \phi-\phi_{S}\right)\right\rangle_{\mathrm{U} \perp}$, and the $2\left\langle\cos \left(2 \phi-\phi_{S}\right)\right\rangle_{\mathrm{L} \perp}$ Fourier amplitudes, do not receive contributions from the longitudinal component of the target polarization. The experimentally measured azimuthal asymmetries are thus only diluted. The correction factor $1 / \cos \theta_{\gamma^{*}}$, however, can be taken as unity under the kinematic conditions here. The second class of exception concerns the $2\left\langle\sin \left(2 \phi-\phi_{S}\right)\right\rangle_{\mathrm{U} \perp}$ and $2\left\langle\cos \left(\phi-\phi_{S}\right)\right\rangle_{\mathrm{L} \perp}$ Fourier amplitudes. The contributions from the longitudinal component to those are equal to the contributions to $2\left\langle\sin \left(2 \phi+\phi_{S}\right)\right\rangle_{\mathrm{U} \perp}$ and $2\left\langle\cos \left(\phi+\phi_{S}\right)\right\rangle_{\mathrm{L} \perp}$. In contrast to the $2\left\langle\sin \left(2 \phi-\phi_{S}\right)\right\rangle_{\mathrm{U} \perp}$ and $2\left\langle\cos \left(\phi-\phi_{S}\right)\right\rangle_{\mathrm{L} \perp}$ Fourier amplitudes, the $2\left\langle\sin \left(2 \phi+\phi_{S}\right)\right\rangle_{\mathrm{U} \perp}$ and $2\left\langle\cos \left(\phi+\phi_{S}\right)\right\rangle_{\mathrm{L} \perp}$ Fourier amplitudes arise solely because of the contribution from the longitudinal component of the target polarization and are thus a measure for that contribution to also $2\left\langle\sin \left(2 \phi-\phi_{S}\right)\right\rangle_{\mathrm{U} \perp}$ and $2\left\langle\cos \left(\phi-\phi_{S}\right)\right\rangle_{\mathrm{L} \perp}$. 


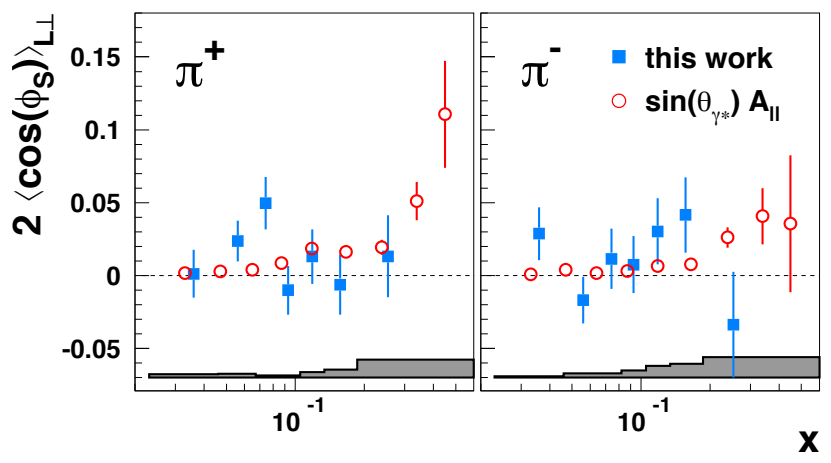

Figure 40. The $x$ dependences of the charged-pion $2\left\langle\cos \left(\phi_{S}\right)\right\rangle_{\mathrm{L} \perp}$ asymmetries measured here (blue squares) and the contribution to this observable from $A_{\|}$(red circles). For the latter, the HERMES measurement [175], which is taken in the range $0.2<z<0.8$, is scaled by the average $\sin \theta_{\gamma^{*}}$ in each bin.

The mixing of target-spin components occurs on the level of the lepton-proton cross sections. Disentangling the contributions thus works in a straightforward way for the CSA by solving the set of linear equations (A.3) as well as (A.5). By contrast, the extraction of SFA already includes a compensation for the $\epsilon$-dependent prefactors, which are in general not the same for the longitudinal and transverse target-spin contributions. As a consequence, a similar separation of the terms from longitudinal and transverse target polarization requires the inclusion of these prefactors in the matrices of eqs (A.3) and (A.5).

\section{B Transverse-momentum factorization and the separation of current and target fragmentation}

This measurement has been performed in the approach presented by Mulders and Tangerman [73] and subsequent works, assuming that the hard scale given by $Q^{2}$ is sufficiently large compared to the transverse momenta involved, and that hadrons are produced in the commonly denoted current or quark fragmentation region [100], i.e., during the hadronization of the quark struck by the virtual photon. In the kinematic region of typical fixedtarget deep-inelastic scattering experiments, the clear separation of the current from the target fragmentation - where the hadron originates from the target remnants (see, e.g., refs. [180, 181]) - is not always granted [101]. As outlined already in section 3.2, the situation is even more vague when looking at transverse-momentum-dependent observables as in this work, because in that case, a hadron produced with large enough transverse momentum in the target fragmentation may mimic a hadron with large transverse momentum from current fragmentation. This complication has attracted increased attention, e.g., through the works of refs. [16, 99]. There is no unique recipe to ensure complete separation of current and target fragmentation and the applicability of QCD factorization theorems may be questioned in the more extreme kinematic regions of growing overlap of the two, e.g., at very low $z$ and large transverse momentum and especially at low values of $Q^{2}$. But where exactly to draw the boundary remains an open issue. 

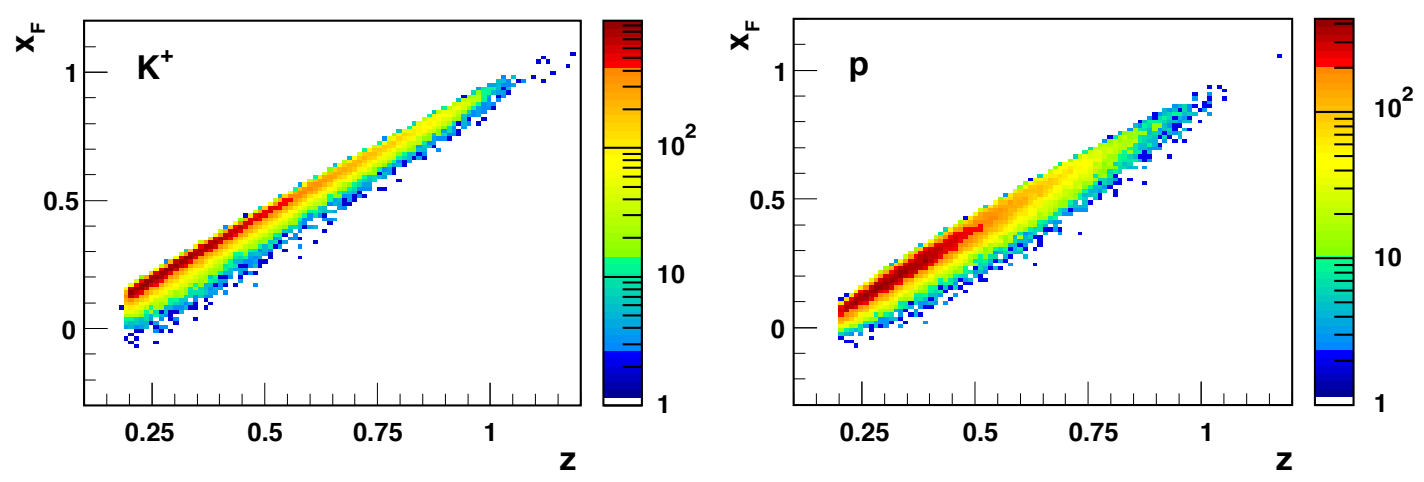

Figure 41. Distributions in $x_{F}$ vs. $z$ of the $K^{+}$(left) and proton (right) yields.

Rather than explicitly applying stringent constraints on the kinematic variables, in this work a large part of the available kinematic phase space is explored within reasonable limits and the azimuthal modulations of interest studied in that kinematic region. In addition, in order to facilitate interpretation of the results, kinematic distributions are provided for the various choices of kinematic binning and hadron species. In this way, the door is open for phenomenology to explore in more detail whether and where the factorized picture might break down for these spin asymmetries.

The particular choice of kinematic distributions provided here are driven by the two aspects considered in the beginning of this section, namely (i) the separation of current and target fragmentation as studied through rapidity distributions, and (ii) the small transverse-momentum requirement as explored by looking at both $Q^{2}$ versus $P_{h \perp}^{2}$ and $Q^{2}$ versus $P_{h \perp}^{2} / z^{2}$.

A presentation in this paper of the distributions for all kinematic bins and hadron species is not practical, they will hence be made available elsewhere (see supplementary material). Instead, a selection of those are presented for the more extreme cases.

\section{B.1 Separation of target and current fragmentation}

In this measurement, hadrons were selected that have a high probability to stem from the current fragmentation. For that a minimum $z$ of 0.2 is required, which predominantly selects forward-going hadrons in the virtual-photon-proton center-of-mass system, forward being the direction of the virtual photon. This is visible in figure 41, where the correlation between $z$ and $x_{F}$ is plotted for both $K^{+}$and protons. For kaons (and likewise pions), $z>0.2$ corresponds to positive $x_{F}$. The situation is slightly less favorable for protons, where still a notable fraction of the yield in the lowest $z$ bin falls in the category of negative $x_{F}$. This can be seen also in the rapidity distributions. They are depicted in figure 42 for the last $x$ bin, while those for pions are shown for the first and last $x$ bin in figure 43. From those distributions it is evident that the majority of events is at forward rapidity. Only a small fraction of events, mainly in the case of protons, populates the region of negative rapidity and do so only for large $P_{h \perp}$ and small $z$. Furthermore, clearly visible in the $\pi^{+}$ 


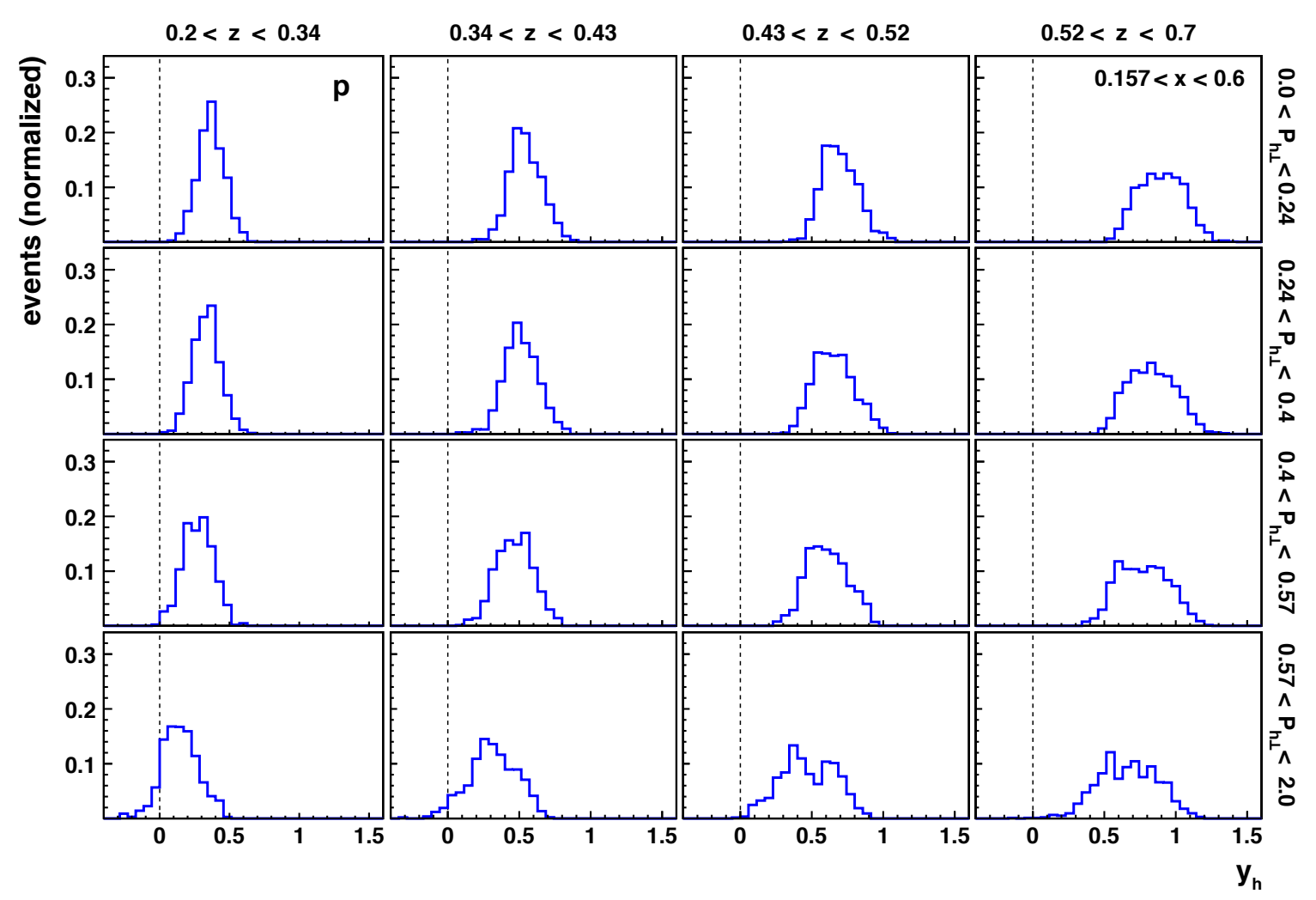

Figure 42. Rapidity distributions for protons in the various $\left(z, P_{h \perp}\right)$ bins of the last $x$ bin. The dashed lines indicate zero rapidity.

figure is a general increase of rapidity with increasing $z$ as well as when decreasing $P_{h \perp}$ and $x$.

\section{B.2 Transverse-momentum versus hard scale}

The interpretation of transverse-momentum-dependent azimuthal distributions in terms of TMD PDFs and FFs as discussed in section 2 requires the presence of one hard scale $\left(Q^{2}\right)$ - which is much larger than a typical nonperturbative-QCD scale like the proton mass or $\Lambda_{\mathrm{QCD}} \cong 0.3 \mathrm{GeV}$, the QCD-scale parameter - and transverse momentum that is small in comparison to $Q^{2}$. Under these conditions, the transverse momentum of the hadron observed can be interpreted as originating from non-pertubative sources in the initial proton structure and in the fragmentation process (including their calculable variations with the hard scale). By contrast, in the region of large transverse momentum, perturbative-QCD radiation is the primary source of the observed transverse momentum of the final-state hadron. This is typically accompanied by a $1 / P_{h \perp}$ suppression of the observable, which usually can be interpreted in terms of collinear PDFs and FFs. In the intermediate region of relatively large transverse momentum but still larger $Q^{2}$, these two descriptions are expected to match their behaviors for a number of azimuthal modulations studied here [182].

In this measurement, $P_{h \perp}$ is of the order of the QCD scale. However, $Q^{2}$ is neither always very large compared to the proton mass nor compared to the transverse momentum. 

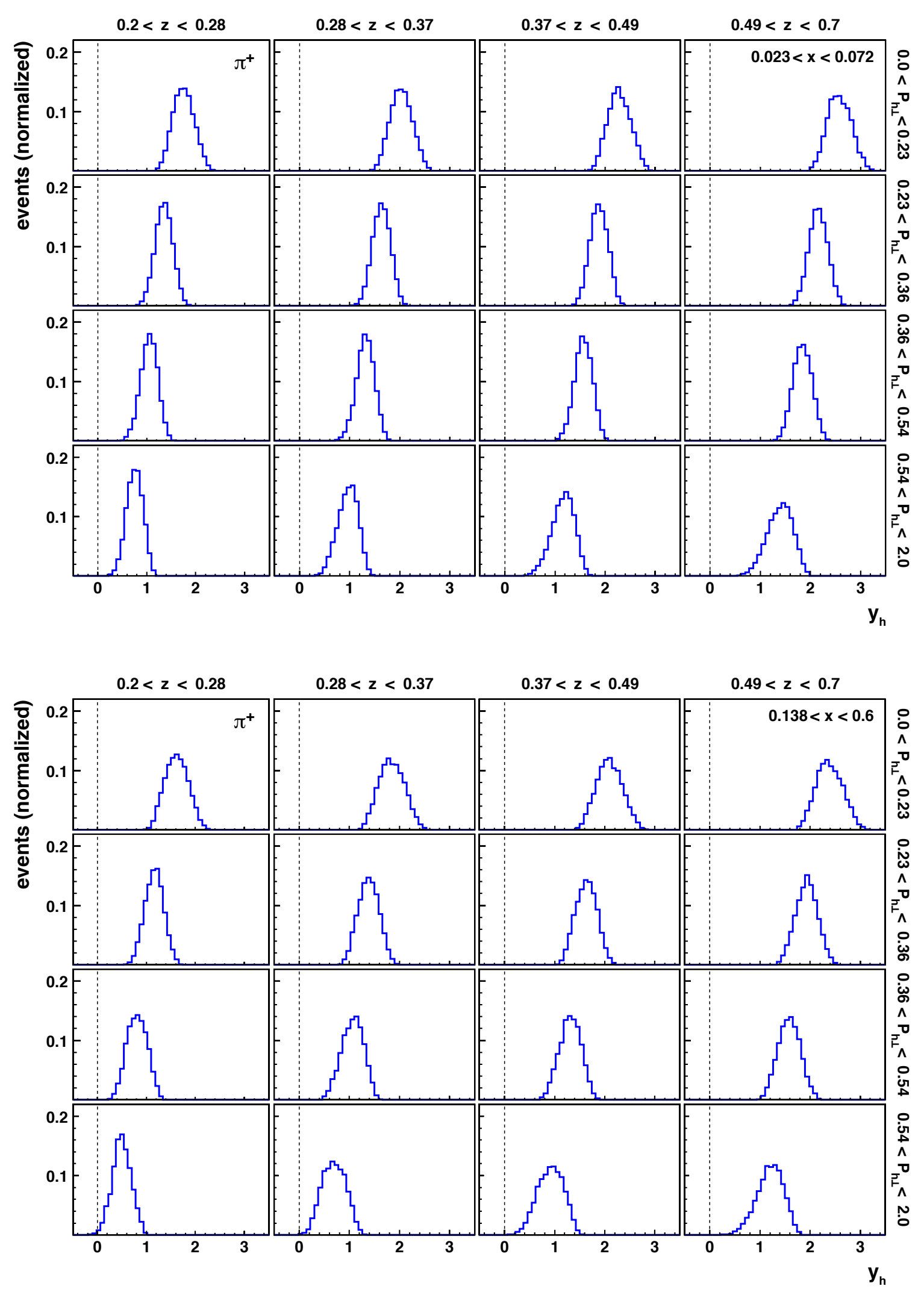

Figure 43. Rapidity distributions for $\pi^{+}$in the various $\left(z, P_{h \perp}\right)$ bins of the first (top) and last (bottom) $x$ bins. The dashed lines indicate zero rapidity. 

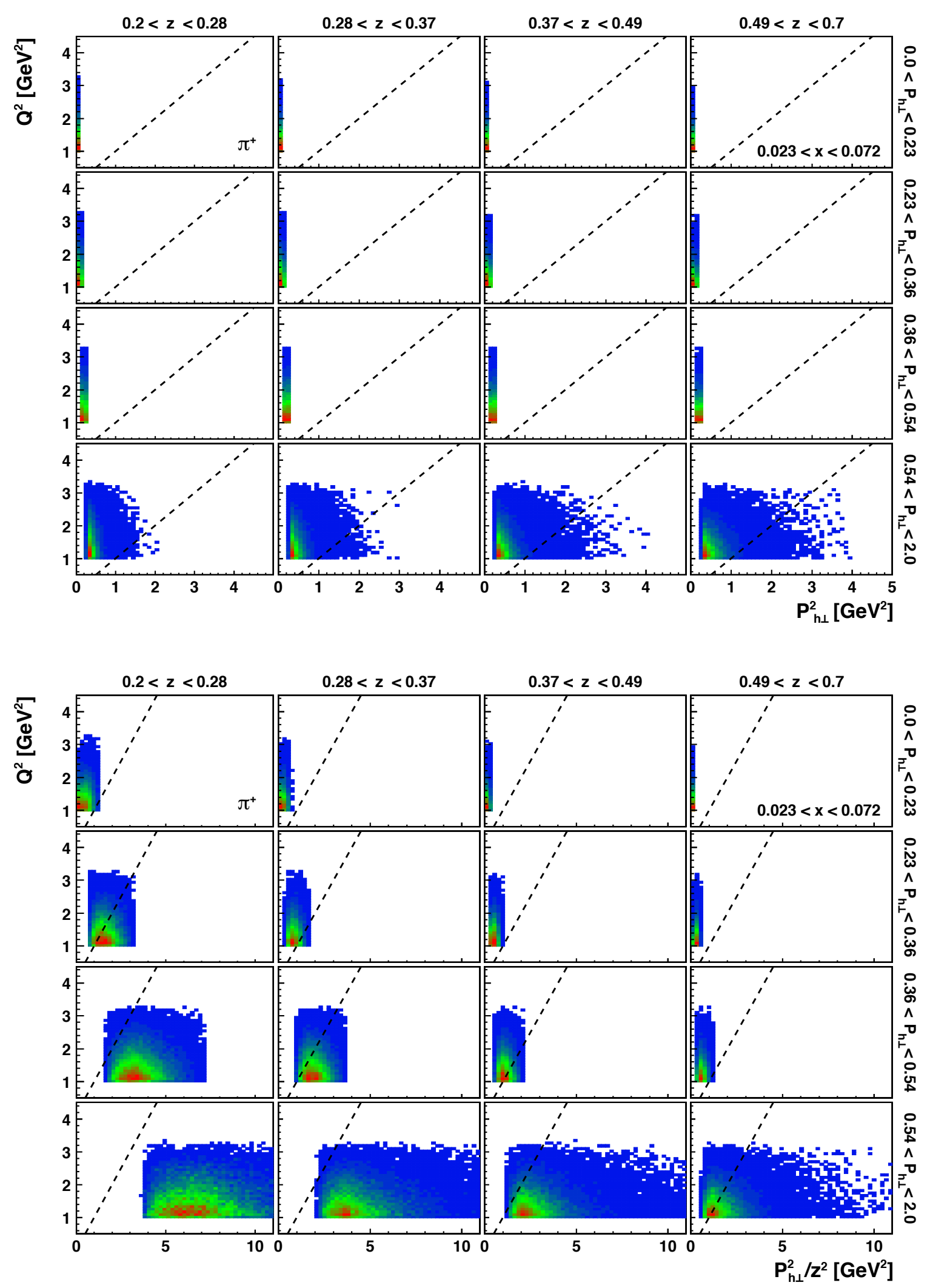

Figure 44. The distribution of $\pi^{+}$events in the $Q^{2}-P_{h \perp}^{2}$ (top) and $Q^{2}-P_{h \perp}^{2} / z^{2}$ (bottom) planes for the various $\left(z, P_{h \perp}\right)$ bins of the lowest $x$ bin. The $Q^{2}=P_{h \perp}^{2}$ (top) and $Q^{2}=P_{h \perp}^{2} / z^{2}$ (bottom) boundaries are indicated by dashed lines. 
Under such conditions, subtleties in the definition of the transverse momentum can also become relevant. One way of testing the requirement of small transverse momentum is comparing directly $P_{h \perp}$ and $Q^{2}$. A different choice of transverse momentum, one that is in particular convenient in factorization proofs of transverse-momentum-dependent processes, is that of the virtual boson in the frame where the two hadrons involved (initial and final in case of semi-inclusive deep-inelastic scattering) are collinear; this choice is commonly denoted as $\mathbf{q}_{T}$. For large enough $Q^{2}, \mathbf{q}_{T}^{2} \simeq P_{h \perp}^{2} / z^{2}$, from which follows the requirement of $P_{h \perp}^{2} / z^{2} \ll Q^{2}$.

In figure 44, the two different transverse momentum scales, $P_{h \perp}^{2}$ and $\mathbf{q}_{T}^{2}$, are compared to $Q^{2}$ for $\pi^{+}$in the $16\left(z, P_{h \perp}\right)$ bins of the lowest $x$ bin. Because $x$ and $Q^{2}$ are highly correlated in this measurement, the lowest $x$ bin corresponds to the region of lowest $Q^{2}$ and hence the region for which the TMD-factorization requirement of small transverse momentum relative to a single hard scale is the more difficult one to fulfill. As visible in the top plot of the figure, for $P_{h \perp}<0.54 \mathrm{GeV}$ all events are above the $Q^{2}=P_{h \perp}^{2}$ diagonal, i.e., the "safe" region. Only in the highest $P_{h \perp}$ bin, a small fraction of events are below that diagonal. For larger values of $x$, the situation is even more favorable with a completely negligible fraction of events in the region of $Q^{2}<P_{h \perp}^{2}$. Even though only presented here for the $\pi^{+}$sample, these observations equally hold for the other hadrons considered in this measurement.

The behavior changes significantly when instead the $Q^{2}$ is plotted against $P_{h \perp}^{2} / z^{2}$, shown in the bottom plot of figure 44 . The requirement of much larger $Q^{2}$ becomes more stringent due the rescaling of the transverse momentum by $1 / z^{2}$, which becomes a large factor for the low- $z$ region. As a consequence, only in the lowest $P_{h \perp}$ bin of the lowest $z$ bin the majority of $\pi^{+}$events fall in the region above the $Q^{2}=P_{h \perp}^{2} / z^{2}$ diagonal. Already in the second $P_{h \perp}$ bin the opposite is the case: most of the events populate the region below that diagonal. Going to bins of larger $P_{h \perp}$ aggravates this situation, up to a point where the majority of events falls in the "unsafe" region for all $z$ bins of the semi-inclusive region. As before, the $\pi^{+}$case is exemplary for all the hadrons considered in this measurement.

The situation improves, as expected from the existing $x-Q^{2}$ correlation, when considering larger values of $x$. This is demonstrated in figures 45 and 46 , where the $Q^{2}$ vs. $P_{h \perp}^{2} / z^{2}$ distributions for $\pi^{+}$are shown for successively increased $x$.

Figure 47 illustrates both the effect of the upper $z$ constraint of 0.7 and of a minimum requirement of $x_{F}>0$ (not applied in this measurement) on the proton-yield distributions as functions of $x, z$, and $P_{h \perp}$. In particular, apart from extending the $z$ spectrum to larger values, there is no visible qualitative change of the various distributions when including the high- $z$ range. On the other hand, data for negative $x_{F}$ are populated in the region of large $P_{h \perp}$ as is expected. This feature of the data is observed for all hadrons, albeit even further suppressed in case of mesons.

\section{C "Polarizing" Pythia6.2 for the estimate of systematic uncertainties}

One of the major challenges of such semi-inclusive measurements as presented here is the evaluation of detector effects, in particular the influence of a finite kinematic acceptance on 

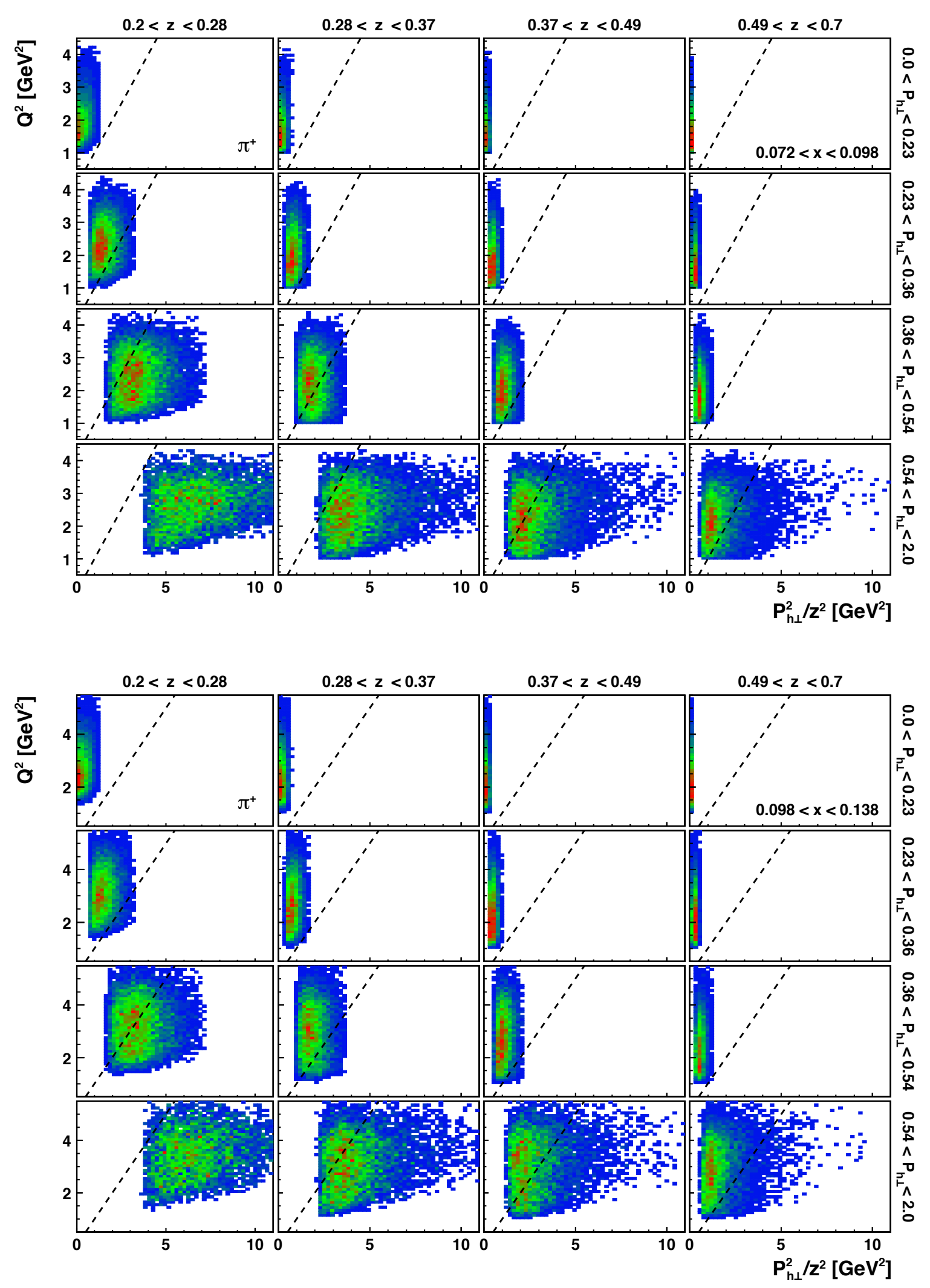

Figure 45. The distribution of $\pi^{+}$events in the $Q^{2}-P_{h \perp}^{2} / z^{2}$ plane for the various $\left(z, P_{h \perp}\right)$ bins of the second (top) and third (bottom) $x$ bin. The $Q^{2}=P_{h \perp}^{2} / z^{2}$ boundaries are indicated by dashed lines. 


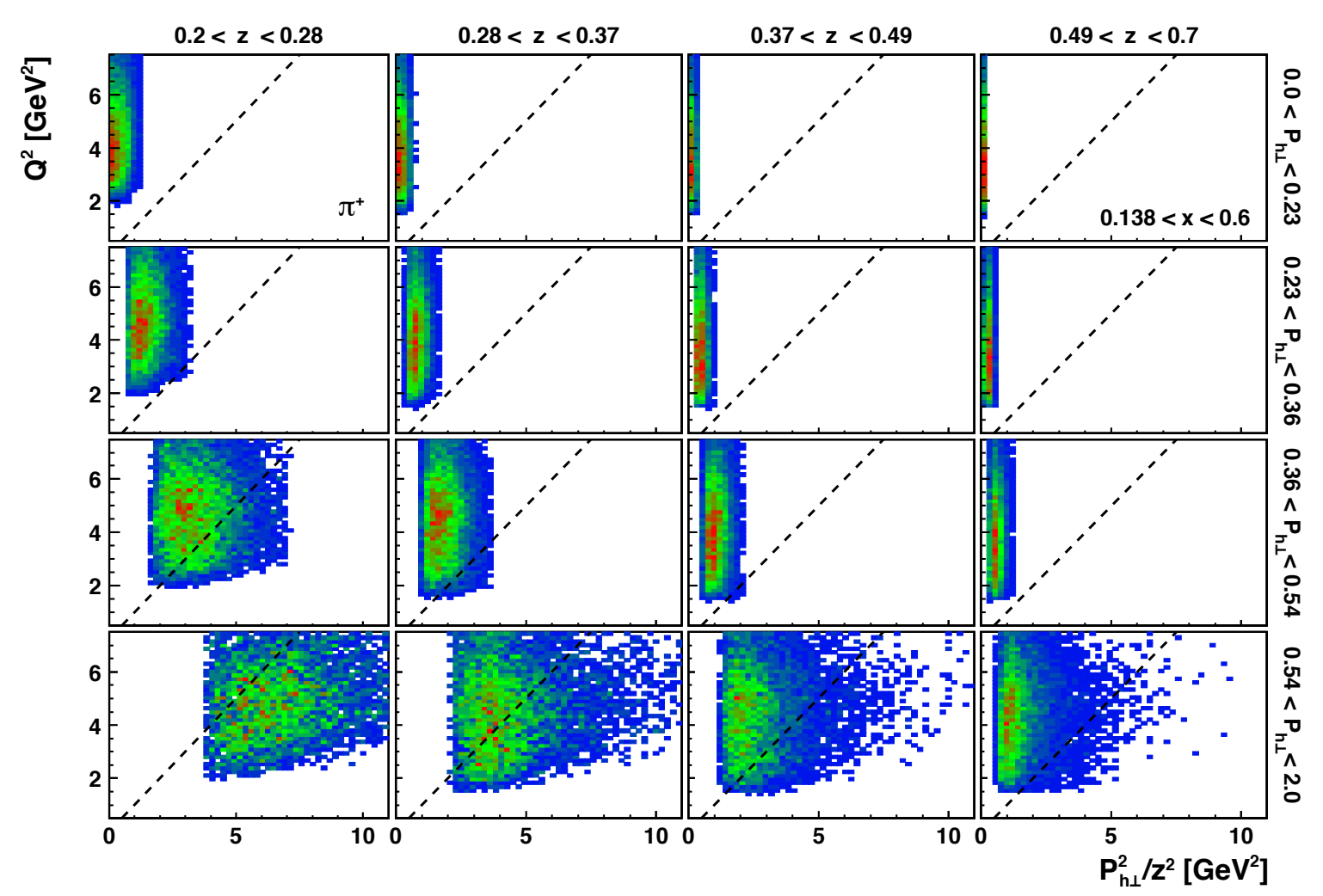

Figure 46. The distribution of $\pi^{+}$events in the $Q^{2}-P_{h \perp}^{2} / z^{2}$ plane for the various $\left(z, P_{h \perp}\right)$ bins of the highest $x$ bin. The $Q^{2}=P_{h \perp}^{2} / z^{2}$ boundaries are indicated by dashed lines.
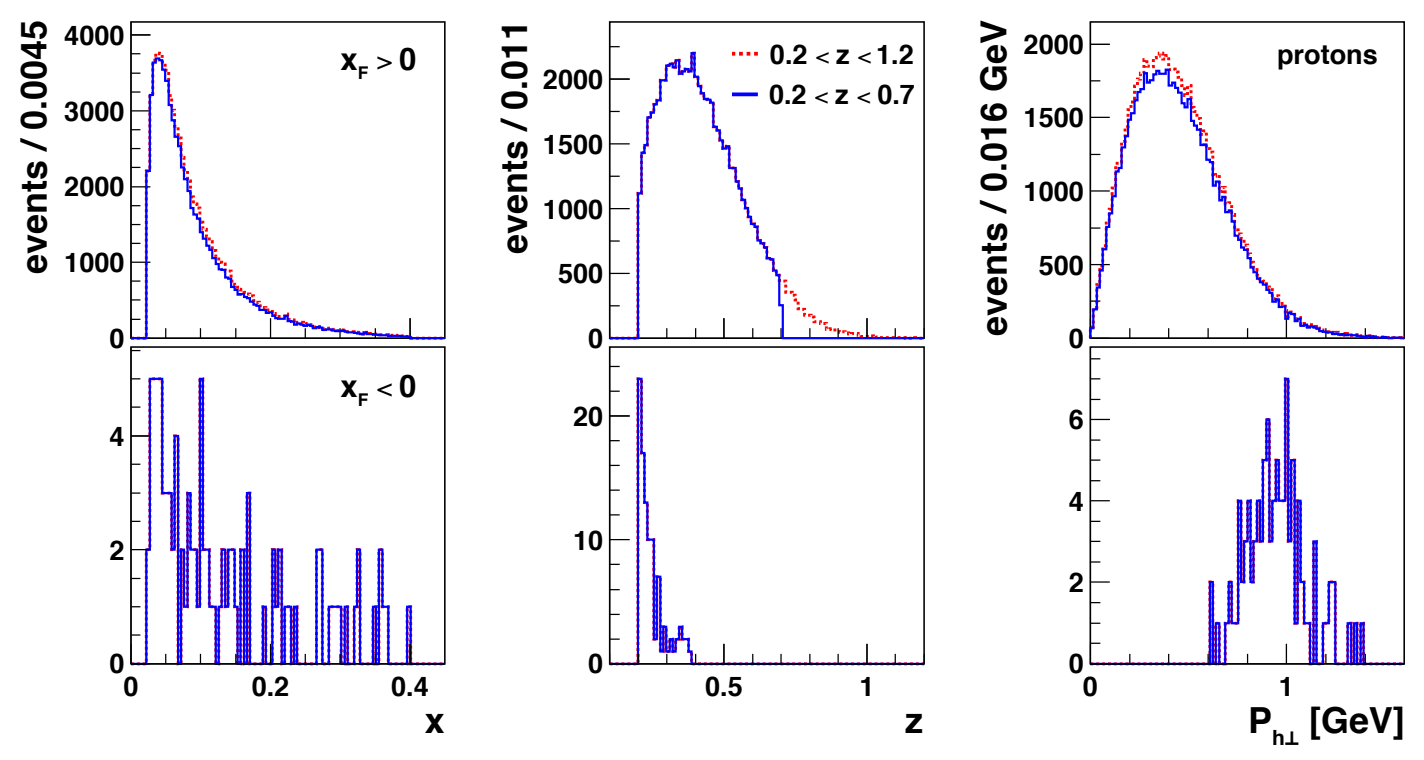

Figure 47. The distribution of proton events versus $x, z$, and $P_{h \perp}$ for both the full range in $z$ of $0.2-1.2$ (red dashed lines) and only the semi-inclusive $z$ range (blue lines). Data with positive $x_{F}$ are presented in the top row. In the bottom, the corresponding distributions for negative $x_{F}$ are shown. 
the Fourier amplitudes extracted. A rigorous analysis procedure involves a fully differential unfolding as done, e.g., for the HERMES measurement of the cosine modulations in the polarization-averaged semi-inclusive deep-inelastic scattering cross section [32]. Here, the limited number of events precludes an unfolding in six dimensions. However, being effectively an asymmetry measurement results in various approximate cancelations of detector effects. Nevertheless, even though the angular Fourier decomposition uses a maximumlikelihood fit unbinned in the azimuthal angles, the limited instrumental acceptance in the remaining kinematic variables can still influence the measurement [106], especially if not performed differential in all the remaining kinematic variables.

Monte Carlo simulations of both the underlying physics as well as of the detector response have become a vital tool for evaluating such systematic effects. The basis for those is a reliable modeling of the experimental setup but also realistic simulations of the physics processes. The measurements presented here enter a territory for which the latter are scarce, mainly due to a lack of knowledge about the various TMDs. Several dedicated physics generators have become available, but none that covers all the TMDs and modulations examined here.

The approach chosen in this analysis makes use of an already very good description of the spin-independent semi-inclusive deep-inelastic scattering cross section provided by Pythia6.2 [96, 97]. Pythia6.2 events come with event weights equal to unity and are hence easy to reshuffle. This is exploited to introduce spin dependence into the otherwise spin-independent event generator $[106,183,184]$. A polarization state $\mathcal{P}$ is assigned to each event $i$ based on a model of the spin asymmetry of interest, e.g.,

$$
\begin{aligned}
\rho<\frac{1}{2}\left[1+\mathcal{A}_{\mathrm{U} \perp}^{\sin \left(\phi-\phi_{S}\right)}\left(\Omega^{i}\right) \sin \left(\phi^{i}-\phi_{S}^{i}\right)\right] & \Rightarrow \mathcal{P}=+1 \\
\rho>\frac{1}{2}\left[1+\mathcal{A}_{\mathrm{U} \perp}^{\sin \left(\phi-\phi_{S}\right)}\left(\Omega^{i}\right) \sin \left(\phi^{i}-\phi_{S}^{i}\right)\right] & \Rightarrow
\end{aligned}
$$

in case of the Sivers Fourier amplitude, by throwing a random variable $0<\rho<1$. Here, $\left(\Omega^{i}, \phi^{i}, \phi_{S}^{i}\right)$ are the fully differential true kinematics for that particular event and $\mathcal{A}_{\mathrm{U} \perp}^{\sin \left(\phi-\phi_{S}\right)}$ is a suitable parameterization for the Sivers modulation. In the specific analysis, eqs. (C.1) and (C.2) are to include all ten azimuthal modulations including the double-spin asymmetries. Virtually any parameterization of the spin dependence can be implemented (as long as fulfilling positivity constraints) without limiting oneself to, e.g., the Gaussian Ansatz for the transverse-momentum dependence. In addition, the full event will remain available, which allows a more thorough study of systematics due to event-topology-dependent detector responses.

Given the scant availability of parameterizations for all modulations studied here, a data-driven approach is employed. An approximate model of reality is obtained by expanding the various Fourier amplitudes measured in a Taylor series in all kinematic variables. A maximum-likelihood fit is employed to extract the coefficients of the fully differential (though truncated) Taylor series for every single azimuthal amplitude appearing in the cross section and for every hadron type. These parameterizations are then used to assign spin states to the Pythia6.2 Monte Carlo simulation - augmented with RAdGeN [107] to account for QED radiative effects and passed through a GEANT3 [108] description of the 
HERMES apparatus (including the RICH particle-identification inefficiencies) - according to eqs. (C.1) and (C.2), with the proper inclusion of all the modulations. The resulting asymmetry amplitudes, reconstructed in the same way as those of the actual HERMES data, are compared to the latter to further tune the truncation of the Taylor series. As an example, in figure 48 (left) a comparison of the fully differential model extracted with the HERMES data is provided for the Collins SFA amplitudes of charged pions. Limitations stemming from the truncation of the Taylor series might be present. While it is not a principle problem to include additional terms, it turns into a more practical problem, especially when attempting to parameterize all spin-dependent terms in the semi-inclusive deep-inelastic scattering cross section, and as a result approaching the usual limit of, e.g., standard Minuit [185], on how many parameters can be determined simultaneously.

In this work most Fourier amplitudes are found to be consistent with zero. In order to keep a finite number of parameters, the following choice was made concerning the parameterization of the fully differential model:

(i) For the three Fourier components that exhibit larger asymmetries and non-linear kinematic dependences (Sivers, Collins, and the $\sin \left(\phi_{S}\right)$ modulation), all the constant and terms linear in $x, z, P_{h \perp}$, and $Q^{2}$ as well as the $2^{\text {nd }}$-order terms in $x, z, P_{h \perp}$, i.e., eleven parameters in total for each modulation, are fit to data.

(ii) For all remaining Fourier components, only the constant and terms linear in $x, z$, $P_{h \perp}$, and $Q^{2}$, i.e., five parameters for each modulation, are included.

The model was expanded around the mean kinematics and fit to data either in the default semi-inclusive range of $0.2<z<0.7$ or in the extended $z$ range. The same model was used for the systematics of both the CSA and SFA and was extracted employing the SFA probability density (3.3) in the maximum-likelihood fit. Variations of the parameterization of the fully differential model were considered and found to give consistent results for these systematic uncertainty.

Antiprotons and neutral pions were treated slightly different due to a lack of statistical precision. More specifically, for the antiproton model, only the standard $0.2<z<0.7$ range is used as there is not a sufficient number of events at larger values of $z$. Furthermore, only the constant and terms linear in $x, z, P_{h \perp}$, and $Q^{2}$ are kept in the Taylor expansions of all ten Fourier amplitudes. The neutral-pion model is constructed using the much better constrained charged-pion models under the assumption of isospin symmetry, i.e.,

$$
\mathcal{A}^{\pi^{0}} \equiv \mathcal{A}^{\text {isospin }}=\frac{\mathcal{A}^{\pi^{+}}+C \cdot \mathcal{A}^{\pi^{-}}}{(1+C)}
$$

where $\mathcal{A}^{\pi^{+}}$and $\mathcal{A}^{\pi^{-}}$are the fully differential models for $\pi^{+}$and $\pi^{-}$, respectively, and the coefficient $C$ represents the ratio of the polarization-averaged semi-inclusive DIS crosssections for negative and positive pion production. In the present analysis the value of $C$ was approximated by

$$
C \equiv \frac{\sigma_{\mathrm{UU}}^{\pi^{-}}}{\sigma_{\mathrm{UU}}^{\pi^{+}}} \approx \frac{\left\langle M^{\pi^{-}}\right\rangle}{\left\langle M^{\pi^{+}}\right\rangle} \approx 0.374
$$



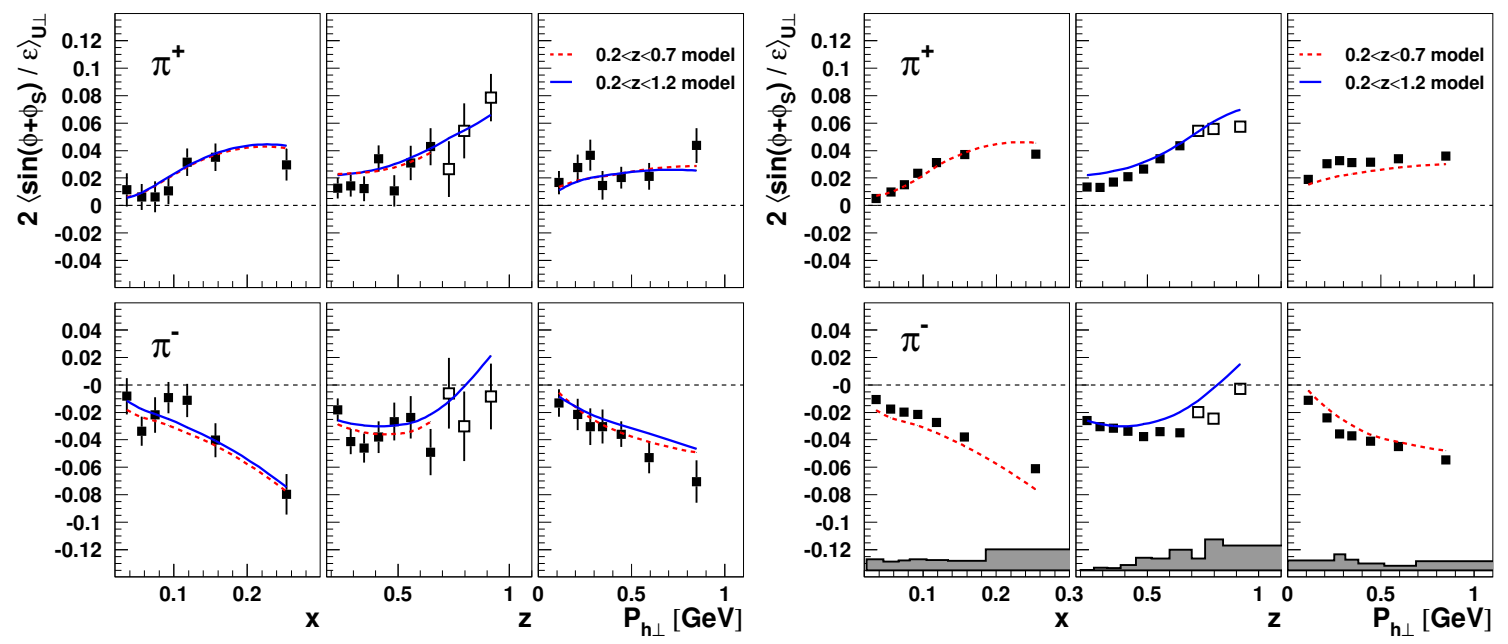

Figure 48. Left: comparison of the HERMES data for charged-pion Collins SFA amplitudes with the fully differential model of those evaluated at the average kinematics of each bin. The dashed curve (red) uses the model based on data in the standard $0.2<z<0.7$ range, while the solid line (blue) includes also the high- $z$ data. Right: comparison of the fully differential models evaluated at the average kinematics of each bin with the fully reconstructed "polarized PYTHIA6.2" simulation (in HERMES acceptance) based on those models. The difference is assigned as systematic uncertainty and shown as uncertainty bands at the bottom of each panel.

using the average, $\left\langle M^{\pi^{ \pm}}\right\rangle$, of the $\pi^{ \pm}$multiplicities [98].

Figures 48 (right) and 49 illustrate the subsequent extraction of systematic uncertainties. The "polarized Pyтнia6.2" events were tracked through a realistic simulation of the experiment and analyzed in the same way as normal experimental data. The reconstructed asymmetry amplitudes are compared to the parameterizations evaluated at the mean reconstructed kinematics of each data point, i.e., in each experimental bin. (This is the same as how the data are usually used in phenomenological fits, e.g., interpreted as the true value of the observable for the average kinematics given alongside.) In each kinematic bin, the difference of the reconstructed Monte Carlo asymmetries and the parameterization, e.g.,

$$
\delta_{\mathrm{sys}}\left(2\left\langle\sin \left(\phi-\phi_{S}\right)\right\rangle_{\mathrm{U} \perp}\right) \equiv\left|2\left\langle\sin \left(\phi-\phi_{S}\right)\right\rangle_{\mathrm{U} \perp}^{\mathrm{MC}}-\mathcal{A}_{\mathrm{U} \perp}^{\sin \left(\phi-\phi_{S}\right)}\left(\langle\Omega\rangle_{\mathrm{bin}}\right)\right|
$$

stems from detector effects including smearing, but more importantly from the integration over kinematic variables, and is assigned as the corresponding systematic uncertainty.

It is worthwhile to highlight that the difference of an average asymmetry in a bin and the asymmetry value at the average kinematics of that bin strongly depends on the non-linearity of the asymmetry and the kinematic region integrated over. That makes the one-dimensional projections much more susceptible to acceptance effects than the threedimensional data presented as the main results in this analysis. 


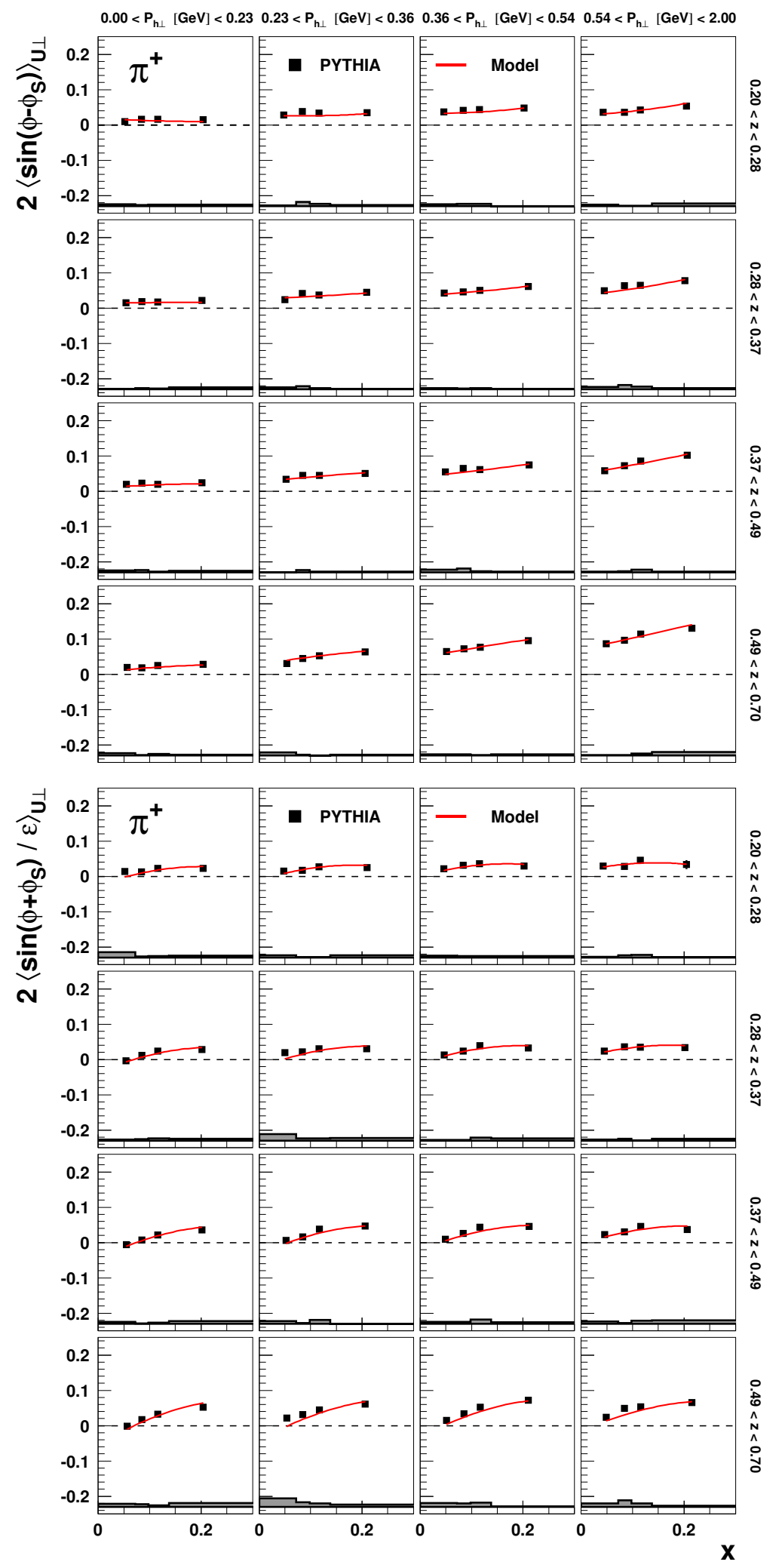

Figure 49. Comparison of the Sivers (top) and Collins (bottom) asymmetries extracted from the "polarized PYTHIA6.2" simulation in the HERMES acceptance with the respective input parameterizations evaluated at the average kinematics of each data point. The difference is assigned as systematic uncertainty and shown as uncertainty bands at the bottom of each panel. 
Open Access. This article is distributed under the terms of the Creative Commons Attribution License (CC-BY 4.0), which permits any use, distribution and reproduction in any medium, provided the original author(s) and source are credited.

\section{References}

[1] M. Anselmino, M. Guidal and P. Rossi, The 3-D Structure of the Nucleon, Eur. Phys. J. A 52 (2016) 164.

[2] J. Gao, L. Harland-Lang and J. Rojo, The structure of the proton in the LHC precision era, Phys. Rept. 742 (2018) 1 [arXiv: 1709.04922] [INSPIRE].

[3] J. Collins, Foundations of perturbative QCD, Cambridge University Press (2013).

[4] R.L. Jaffe, Spin, Twist and Hadron Structure in Deep Inelastic Processes, in F. Lenz, H. Grießhammer and D. Stoll eds., Lectures on QCD: Applications, vol. 496 of Lecture Notes in Physics, Springer, Berlin, New York (1997) pp. 178-249.

[5] M. Engelhardt et al., Quark orbital angular momentum in the proton evaluated using a direct derivative method, PoS LATTICE2018 (2018) 115 [arXiv:1901.00843] [INSPIRE].

[6] C. Alexandrou et al., Complete flavor decomposition of the spin and momentum fraction of the proton using lattice QCD simulations at physical pion mass, Phys. Rev. D 101 (2020) 094513 [arXiv: 2003.08486] [INSPIRE].

[7] A. Bacchetta, U. D'Alesio, M. Diehl and C. Miller, Single-spin asymmetries: The Trento conventions, Phys. Rev. D 70 (2004) 117504 [hep-ph/0410050] [InSPIRE].

[8] A. Bacchetta, M. Diehl, K. Goeke, A. Metz, P.J. Mulders and M. Schlegel, Semi-inclusive deep inelastic scattering at small transverse momentum, JHEP 02 (2007) 093 [hep-ph/0611265] [INSPIRE].

[9] J.C. Collins and D.E. Soper, Parton distribution and decay functions, Nucl. Phys. B 194 (1982) 445 [INSPIRE].

[10] J.C. Collins, D.E. Soper and G.F. Sterman, Transverse momentum distribution in Drell-Yan pair and $W$ and $Z$ boson production, Nucl. Phys. B 250 (1985) 199 [INSPIRE].

[11] J.C. Collins, D.E. Soper and G.F. Sterman, Factorization of Hard Processes in QCD, Adv. Ser. Direct. High Energy Phys. 5 (1989) 1 [hep-ph/0409313] [INSPIRE].

[12] X. Ji, J.-P. Ma and F. Yuan, QCD factorization for semi-inclusive deep-inelastic scattering at low transverse momentum, Phys. Rev. D 71 (2005) 034005 [hep-ph/0404183] [INSPIRE].

[13] M.G. Echevarría, A. Idilbi and I. Scimemi, Soft and Collinear Factorization and Transverse Momentum Dependent Parton Distribution Functions, Phys. Lett. B 726 (2013) 795 [arXiv:1211.1947] [INSPIRE].

[14] T.C. Rogers, An overview of transverse-momentum-dependent factorization and evolution, Eur. Phys. J. A 52 (2016) 153 [arXiv:1509.04766] [InSPIRE].

[15] J. Collins and T.C. Rogers, Connecting different TMD factorization formalisms in QCD, Phys. Rev. D 96 (2017) 054011 [arXiv:1705.07167] [INSPIRE].

[16] M. Boglione et al., Mapping the kinematical regimes of semi-inclusive deep inelastic scattering, JHEP 10 (2019) 122 [arXiv:1904.12882] [INSPIRE].

[17] J.C. Collins and D.E. Soper, Back-to-back jets in QCD, Nucl. Phys. B 193 (1981) 381 [Erratum ibid. 213 (1983) 545] [INSPIRE].

[18] S. Aybat and T.C. Rogers, Transverse momentum dependent parton distribution and fragmentation functions with QCD evolution, Phys. Rev. D 83 (2011) 114042 [arXiv:1101.5057] [INSPIRE]. 
[19] M.G. Echevarría, A. Idilbi, A. Schäfer and I. Scimemi, Model independent evolution of transverse momentum dependent distribution functions (TMDs) at NNLL, Eur. Phys. J. C 73 (2013) 2636 [arXiv:1208.1281] [INSPIRE].

[20] A. Bacchetta, F. Delcarro, C. Pisano, M. Radici and A. Signori, Extraction of partonic transverse momentum distributions from semi-inclusive deep-inelastic scattering, Drell-Yan and Z-boson production, JHEP 06 (2017) 081 [Erratum ibid. 06 (2019) 051] [arXiv:1703.10157] [INSPIRE].

[21] A. Bacchetta et al., Transverse-momentum-dependent parton distributions up to $N^{3} L L$ from Drell-Yan data, JHEP 07 (2020) 117 [arXiv:1912.07550] [INSPIRE].

[22] I. Scimemi and A. Vladimirov, Non-perturbative structure of semi-inclusive deep-inelastic and Drell-Yan scattering at small transverse momentum, JHEP 06 (2020) 137 [arXiv: 1912.06532] [INSPIRE].

[23] X. Ji, Y. Liu and Y.-S. Liu, TMD soft function from large-momentum effective theory, Nucl. Phys. B 955 (2020) 115054 [arXiv:1910.11415] [INSPIRE].

[24] A.A. Vladimirov and A. Schäfer, Transverse-momentum-dependent factorization for lattice observables, Phys. Rev. D 101 (2020) 074517 [arXiv:2002.07527] [INSPIRE].

[25] P. Shanahan, M. Wagman and Y. Zhao, Collins-Soper kernel for TMD evolution from lattice QCD, Phys. Rev. D 102 (2020) 014511 [arXiv:2003.06063] [INSPIRE].

[26] R.L. Jaffe and X. Ji, Chiral odd parton distributions and polarized Drell-Yan, Phys. Rev. Lett. 67 (1991) 552 [inSPIRE].

[27] X. Artru and M. Mekhfi, Transversely polarized parton densities, their evolution and their measurement, Z. Phys. C 45 (1990) 669 [InSPIRE].

[28] HERMES collaboration, Single-spin Asymmetries in Semi-Inclusive Deep-Inelastic Scattering on a Transversely Polarized Hydrogen Target, Phys. Rev. Lett. 94 (2005) 012002 [hep-ex/0408013] [INSPIRE].

[29] HERMES collaboration, Effects of transversity in deep-inelastic scattering by polarized protons, Phys. Lett. B 693 (2010) 11 [arXiv:1006.4221] [InSPIRE].

[30] D.W. Sivers, Single-spin production asymmetries from the hard scattering of pointlike constituents, Phys. Rev. D 41 (1990) 83 [INSPIRE].

[31] D. Boer and P.J. Mulders, Time-reversal odd distribution functions in leptoproduction, Phys. Rev. D 57 (1998) 5780 [hep-ph/9711485] [inSPIRE].

[32] HERMES collaboration, Azimuthal distributions of charged hadrons, pions, and kaons produced in deep-inelastic scattering off unpolarized protons and deuterons, Phys. Rev. D 87 (2013) 012010 [arXiv: 1204.4161] [INSPIRE].

[33] COMPASS collaboration, Measurement of azimuthal hadron asymmetries in semi-inclusive deep inelastic scattering off unpolarised nucleons, Nucl. Phys. B 886 (2014) 1046 [arXiv:1401.6284] [INSPIRE].

[34] J. Antille et al., Spin dependence of the inclusive reaction $p+p$ (polarized) $\rightarrow \pi^{0}+X$ at $24 \mathrm{GeV} / \mathrm{c}$ for high-p ${ }_{T} \pi^{0}$ produced in the central region, Phys. Lett. B 94 (1980) 523 [INSPIRE].

[35] J.C. Collins, Leading-twist single-transverse-spin asymmetries: Drell-Yan and deep-inelastic scattering, Phys. Lett. B 536 (2002) 43 [hep-ph/0204004] [InSPIRE].

[36] S.J. Brodsky, D.S. Hwang and I. Schmidt, Final-state interactions and single-spin asymmetries in semi-inclusive deep inelastic scattering, Phys. Lett. B 530 (2002) 99 [hep-ph/0201296] [INSPIRE]. 
[37] X. Ji and F. Yuan, Parton distributions in light-cone gauge: where are the final-state interactions?, Phys. Lett. B 543 (2002) 66 [hep-ph/0206057] [INSPIRE].

[38] A.V. Belitsky, X. Ji and F. Yuan, Final state interactions and gauge invariant parton distributions, Nucl. Phys. B 656 (2003) 165 [hep-ph/0208038] [inSPIRE].

[39] M. Burkardt, Chromodynamic lensing and transverse single spin asymmetries, Nucl. Phys. A 735 (2004) 185 [hep-ph/0302144] [INSPIRE].

[40] HERMES collaboration, Observation of the Naive-T-odd Sivers Effect in Deep-Inelastic Scattering, Phys. Rev. Lett. 103 (2009) 152002 [arXiv:0906.3918] [INSPIRE].

[41] COMPASS collaboration, Measurement of the Collins and Sivers asymmetries on transversely polarised protons, Phys. Lett. B 692 (2010) 240 [arXiv: 1005.5609] [InSPIRE].

[42] STAR collaboration, Measurement of the Transverse Single-Spin Asymmetry in $p^{\uparrow}+p \rightarrow W^{ \pm} / Z^{0}$ at RHIC, Phys. Rev. Lett. 116 (2016) 132301 [arXiv:1511.06003] [INSPIRE].

[43] COMPASS collaboration, First Measurement of Transverse-Spin-Dependent Azimuthal Asymmetries in the Drell-Yan Process, Phys. Rev. Lett. 119 (2017) 112002 [arXiv: 1704.00488] [INSPIRE].

[44] M. Burkardt and G. Schnell, Anomalous magnetic moments and quark orbital angular momentum, Phys. Rev. D 74 (2006) 013002 [hep-ph/0510249] [INSPIRE].

[45] M. Burkardt, Impact parameter dependent parton distributions and transverse single spin asymmetries, Phys. Rev. D 66 (2002) 114005 [hep-ph/0209179] [INSPIRE].

[46] A. Bacchetta and M. Radici, Constraining Quark Angular Momentum Through Semi-Inclusive Measurements, Phys. Rev. Lett. 107 (2011) 212001 [arXiv:1107.5755] [INSPIRE].

[47] B. Pasquini, S. Rodini and A. Bacchetta, Revisiting model relations between T-odd transverse-momentum dependent parton distributions and generalized parton distributions, Phys. Rev. D 100 (2019) 054039 [arXiv:1907.06960] [INSPIRE].

[48] R.D. Tangerman and P.J. Mulders, Probing transverse quark polarization in deep-inelastic leptoproduction, Phys. Lett. B 352 (1995) 129 [hep-ph/9501202] [INSPIRE].

[49] D. Gutiérrez-Reyes, I. Scimemi and A.A. Vladimirov, Twist-2 matching of transverse momentum dependent distributions, Phys. Lett. B 769 (2017) 84 [arXiv:1702.06558] [INSPIRE].

[50] D. Gutierrez-Reyes, I. Scimemi and A. Vladimirov, Transverse momentum dependent transversely polarized distributions at next-to-next-to-leading-order, JHEP 07 (2018) 172 [arXiv: 1805.07243] [INSPIRE].

[51] H. Avakian, A.V. Efremov, P. Schweitzer and F. Yuan, Transverse momentum dependent distribution function $h_{1 T}^{\perp}$ and the single spin asymmetry $A_{U T}^{\sin \left(3 \phi-\phi_{S}\right)}$, Phys. Rev. D 78 (2008) 114024 [arXiv:0805.3355] [INSPIRE].

[52] G.A. Miller, Densities, parton distributions, and measuring the nonspherical shape of the nucleon, Phys. Rev. C $\mathbf{7 6}$ (2007) 065209 [arXiv:0708.2297] [InSPIRE].

[53] M. Burkardt, Spin-orbit correlations and single-spin asymmetries, in A. Radyushkin and P. Stoler eds., Exclusive Reactions at High Momentum Transfer, World Scientific (2008), pp. 78-86 [arXiv: 0709.2966] [INSPIRE].

[54] A. Kotzinian, New quark distributions and semi-inclusive electroproduction on polarized nucleons, Nucl. Phys. B 441 (1995) 234 [hep-ph/9412283] [INSPIRE]. 
[55] Jefferson LaB Hall A collaboration, Measurement of "pretzelosity" asymmetry of charged pion production in semi-inclusive deep inelastic scattering on a polarized ${ }^{3}$ He target, Phys. Rev. C 90 (2014) 055209 [arXiv:1312.3047] [inSPIRE].

[56] COMPASS collaboration, Sivers asymmetry extracted in SIDIS at the hard scales of the Drell-Yan process at COMPASS, Phys. Lett. B $\mathbf{7 7 0}$ (2017) 138 [arXiv:1609.07374] [INSPIRE].

[57] J.P. Ralston and D.E. Soper, Production of dimuons from high-energy polarized proton-proton collisions, Nucl. Phys. B 152 (1979) 109 [INSPIRE].

[58] A.M. Kotzinian and P.J. Mulders, Longitudinal quark polarization in transversely polarized nucleons, Phys. Rev. D 54 (1996) 1229 [hep-ph/9511420] [INSPIRE].

[59] M. Diehl and P. Hägler, Spin densities in the transverse plane and generalized transversity distributions, Eur. Phys. J. C 44 (2005) 87 [hep-ph/0504175] [InSPIRE].

[60] S. Boffi, A.V. Efremov, B. Pasquini and P. Schweitzer, Azimuthal spin asymmetries in light-cone constituent quark models, Phys. Rev. D 79 (2009) 094012 [arXiv:0903.1271] [INSPIRE].

[61] A. Bacchetta and A. Prokudin, Evolution of the helicity and transversity. Transverse-momentum-dependent parton distributions, Nucl. Phys. B 875 (2013) 536 [arXiv: 1303.2129] [INSPIRE].

[62] H. Avakian, A.V. Efremov, K. Goeke, A. Metz, P. Schweitzer and T. Teckentrup, Are there approximate relations among transverse momentum dependent distribution functions?, Phys. Rev. D 77 (2008) 014023 [arXiv:0709.3253] [InSPIRE].

[63] S. Wandzura and F. Wilczek, Sum rules for spin-dependent electroproduction: test of relativistic constituent quarks, Phys. Lett. B 72 (1977) 195 [InSPIRE].

[64] S. Bastami et al., Semi-inclusive deep-inelastic scattering in Wandzura-Wilczek-type approximation, JHEP 06 (2019) 007 [arXiv:1807.10606] [INSPIRE].

[65] A. Accardi, A. Bacchetta, W. Melnitchouk and M. Schlegel, What can break the Wandzura-Wilczek relation?, JHEP 11 (2009) 093 [arXiv:0907.2942] [INSPIRE].

[66] Jefferson Lab Hall A collaboration, Beam-Target Double-Spin Asymmetry $A_{L T}$ in Charged Pion Production from Deep Inelastic Scattering on a Transversely Polarized ${ }^{3} \mathrm{He}$ Target at 1.4<Q $Q^{2}<2.7 \mathrm{GeV}^{2}$, Phys. Rev. Lett. 108 (2012) 052001 [arXiv:1108.0489] [INSPIRE].

[67] HERMES collaboration, Evidence for a Single-Spin Azimuthal Asymmetry in Semi-inclusive Pion Electroproduction, Phys. Rev. Lett. 84 (2000) 4047 [hep-ex/9910062] [INSPIRE].

[68] HERMES collaboration, Single-spin azimuthal asymmetries in electroproduction of neutral pions in semi-inclusive deep-inelastic scattering, Phys. Rev. D 64 (2001) 097101 [hep-ex/0104005] [INSPIRE].

[69] HERMES collaboration, Measurement of single-spin azimuthal asymmetries in semi-inclusive electroproduction of pions and kaons on a longitudinally polarised deuterium target, Phys. Lett. B 562 (2003) 182 [hep-ex/0212039] [INSPIRE].

[70] HERMES collaboration, Subleading-twist effects in single-spin asymmetries in semi-inclusive deep-inelastic scattering on a longitudinally polarized hydrogen target, Phys. Lett. B 622 (2005) 14 [hep-ex/0505042] [INSPIRE].

[71] HERMES collaboration, Beam-spin asymmetries in the azimuthal distribution of pion electroproduction, Phys. Lett. B 648 (2007) 164 [hep-ex/0612059] [INSPIRE]. 
[72] HERMES collaboration, Beam-helicity asymmetries for single-hadron production in semi-inclusive deep-inelastic scattering from unpolarized hydrogen and deuterium targets, Phys. Lett. B 797 (2019) 134886 [arXiv:1903.08544] [InSPIRE].

[73] P.J. Mulders and R.D. Tangerman, The complete tree-level result up to order $1 / Q$ for polarized deep-inelastic leptoproduction, Nucl. Phys. B 461 (1996) 197 [Erratum ibid. 484 (1997) 538] [hep-ph/9510301] [INSPIRE].

[74] N. Christ and T.D. Lee, Possible Tests of $C_{s t}$ and $T_{\text {st }}$ Invariances in $l^{ \pm}+N \rightarrow l^{ \pm}+\Gamma$ and $A \rightarrow B+e^{+}+e^{-}$, Phys. Rev. 143 (1966) 1310 [inSPIRE].

[75] HERMES collaboration, Search for a two-photon exchange contribution to inclusive deep-inelastic scattering, Phys. Lett. B 682 (2010) 351 [arXiv:0907.5369] [INSPIRE].

[76] F. Yuan and J. Zhou, Collins Fragmentation and the Single Transverse Spin Asymmetry, Phys. Rev. Lett. 103 (2009) 052001 [arXiv:0903.4680] [InSPIRE].

[77] K. Kanazawa, Y. Koike, A. Metz, D. Pitonyak and M. Schlegel, Operator constraints for twist-3 functions and Lorentz invariance properties of twist-3 observables, Phys. Rev. D 93 (2016) 054024 [arXiv: 1512.07233] [INSPIRE].

[78] E.C. Aschenauer, U. D'Alesio and F. Murgia, TMDs and SSAs in hadronic interactions, Eur. Phys. J. A 52 (2016) 156 [arXiv:1512.05379] [inSPIRE].

[79] L. Gamberg, Z.-B. Kang, A. Metz, D. Pitonyak and A. Prokudin, Left-right spin asymmetry in $\ell N^{\uparrow} \rightarrow h X$, Phys. Rev. D 90 (2014) 074012 [arXiv: 1407.5078] [InSPIRE].

[80] HERMES collaboration, Transverse target single-spin asymmetry in inclusive electroproduction of charged pions and kaons, Phys. Lett. B 728 (2014) 183 [arXiv:1310.5070] [INSPIRE].

[81] Jefferson Lab Hall A collaboration, Single spin asymmetries of inclusive hadrons produced in electron scattering from a transversely polarized ${ }^{3}$ He target, Phys. Rev. C 89 (2014) 042201 [arXiv: 1311.1866] [INSPIRE].

[82] R.L. Jaffe and X. Ji, Novel quark fragmentation functions and the nucleon's transversity distribution, Phys. Rev. Lett. 71 (1993) 2547 [hep-ph/9307329] [INSPIRE].

[83] A.V. Efremov, L. Mankiewicz and N.A. Törnqvist, Jet handedness as a measure of quark and gluon polarization, Phys. Lett. B 284 (1992) 394 [INSPIRE].

[84] J.C. Collins, S.F. Heppelmann and G.A. Ladinsky, Measuring transversity densities in singly polarized hadron-hadron and lepton-hadron collisions, Nucl. Phys. B 420 (1994) 565 [hep-ph/9305309] [INSPIRE].

[85] HERMES collaboration, Measurement of the virtual-photon asymmetry $A_{2}$ and the spin-structure function $g_{2}$ of the proton, Eur. Phys. J. C 72 (2012) 1921 [arXiv: 1112.5584] [INSPIRE].

[86] SpIn Muon collaboration, Spin structure of the proton from polarized inclusive deep-inelastic muon-proton scattering, Phys. Rev. D 56 (1997) 5330 [hep-ex/9702005] [INSPIRE].

[87] E143 collaboration, Measurements of the proton and deuteron spin structure functions $g_{1}$ and $g_{2}$, Phys. Rev. D 58 (1998) 112003 [hep-ph/9802357] [InSPIRE].

[88] E155 collaboration, Precision measurement of the proton and deuteron spin structure functions $g_{2}$ and asymmetries $A_{2}$, Phys. Lett. B 553 (2003) 18 [hep-ex/0204028] [INSPIRE].

[89] SANE collaboration, Revealing Color Forces with Transverse Polarized Electron Scattering, Phys. Rev. Lett. 122 (2019) 022002 [arXiv: 1805.08835] [INSPIRE].

[90] M. Burkardt, Transverse force on quarks in deep-inelastic scattering, Phys. Rev. D 88 (2013) 114502 [arXiv:0810.3589] [INSPIRE]. 
[91] F.P. Aslan, M. Burkardt and M. Schlegel, Transverse force tomography, Phys. Rev. D 100 (2019) 096021 [arXiv : 1904.03494] [inSPIRE].

[92] HERMES collaboration, The HERMES Spectrometer, Nucl. Instrum. Meth. A 417 (1998) 230 [hep-ex/9806008] [INSPIRE].

[93] HERMES collaboration, The HERMES polarized hydrogen and deuterium gas target in the HERA electron storage ring, Nucl. Instrum. Meth. A 540 (2005) 68 [physics/0408137] [INSPIRE].

[94] A.A. Sokolov and I.M. Ternov, On polarization and spin effects in the theory of synchrotron radiation, Sov. Phys. Dokl. 8 (1964) 1203 [INSPIRE].

[95] N. Akopov et al., The HERMES dual-radiator ring imaging Cherenkov detector, Nucl. Instrum. Meth. A 479 (2002) 511 [physics/0104033] [INSPIRE].

[96] T. Sjöstrand et al., High-energy physics event generation with PYTHIA 6.1, Comput. Phys. Commun. 135 (2001) 238 [hep-ph/0010017] [INSPIRE].

[97] T. Sjöstrand, L. Lönnblad and S. Mrenna, PYthiA 6.2: Physics and Manual, hep-ph/0108264 [INSPIRE].

[98] HERMES collaboration, Multiplicities of charged pions and kaons from semi-inclusive deep-inelastic scattering by the proton and the deuteron, Phys. Rev. D 87 (2013) 074029 [arXiv: 1212.5407] [INSPIRE].

[99] M. Boglione, J. Collins, L. Gamberg, J.O. Gonzalez-Hernandez, T.C. Rogers and N. Sato, Kinematics of current region fragmentation in semi-inclusive deeply inelastic scattering, Phys. Lett. B 766 (2017) 245 [arXiv:1611.10329] [INSPIRE].

[100] E.L. Berger, Semi-inclusive inelastic electron scattering from nuclei, in proceedings of NPAS Workshop on Electronuclear Physics with Internal Targets, SLAC, 5-8 January 1987, pp. $82-91$.

[101] P.J. Mulders, Current fragmentation in semiinclusive leptoproduction, AIP Conf. Proc. $\mathbf{5 8 8}$ (2001) 75 [hep-ph/0010199] [INSPIRE].

[102] M. Diehl and S. Sapeta, On the analysis of lepton scattering on longitudinally or transversely polarized protons, Eur. Phys. J. C 41 (2005) 515 [hep-ph/0503023] [InSPIRE].

[103] F.T. Solmitz, Analysis of experiments in particle physics, Annu. Rev. Nucl. Sci. 14 (1964) 375 .

[104] W. Weibull, A Statistical Distribution Function of Wide Applicability, J. Appl. Mech. 18 (1951) 293.

[105] R.N. Cahn, Azimuthal dependence in leptoproduction: A simple parton model calculation, Phys. Lett. B 78 (1978) 269 [INSPIRE].

[106] G. Schnell, Monte Carlo methods for TMD analyses, EPJ Web Conf. 85 (2015) 02024 [INSPIRE].

[107] I. Akushevich, H. Böttcher and D. Ryckbosch, RADGEN 1.0: Monte Carlo Generator for Radiative Events in DIS on Polarized and Unpolarized Targets, in Workshop on Monte Carlo Generators for HERA Physics (Plenary Starting Meeting), pp. 554-565, 4, 1998, hep-ph/9906408 [INSPIRE].

[108] R. Brun, R. Hagelberg, M. Hansroul and J.C. Lassalle, Geant: Simulation Program for Particle Physics Experiments. User Guide and Reference Manual, (1978), InSPIRE.

[109] R. Brun, F. Bruyant, M. Maire, A.C. McPherson and P. Zanarini, GEANT3, (1987), INSPIRE. 
[110] M. Anselmino et al., Transversity and Collins functions from SIDIS and $e^{+} e^{-}$data, Phys. Rev. D 75 (2007) 054032 [hep-ph/0701006] [INSPIRE].

[111] M. Anselmino, M. Boglione, U. D’Alesio, S. Melis, F. Murgia and A. Prokudin, Simultaneous extraction of transversity and Collins functions from new semi-inclusive deep inelastic scattering and $e^{+} e^{-}$data, Phys. Rev. D 87 (2013) 094019 [arXiv:1303.3822] [INSPIRE].

[112] M. Anselmino et al., Collins functions for pions from SIDIS and new $e^{+} e^{-}$data: A first glance at their transverse momentum dependence, Phys. Rev. D 92 (2015) 114023 [arXiv: 1510.05389] [INSPIRE].

[113] H.-W. Lin, W. Melnitchouk, A. Prokudin, N. Sato and H. Shows, First Monte Carlo Global Analysis of Nucleon Transversity with Lattice QCD Constraints, Phys. Rev. Lett. 120 (2018) 152502 [arXiv:1710.09858] [INSPIRE].

[114] V. Barone et al., Transversity distributions from difference asymmetries in semi-inclusive DIS, Phys. Rev. D 99 (2019) 114004 [arXiv:1902.08445] [InSPIRE].

[115] U. D'Alesio, C. Flore and A. Prokudin, Role of the Soffer bound in determination of transversity and the tensor charge, Phys. Lett. B 803 (2020) 135347 [arXiv:2001.01573] [INSPIRE].

[116] Jefferson Lab Angular Momentum collaboration, Origin of single transverse-spin asymmetries in high-energy collisions, Phys. Rev. D 102 (2020) 054002 [arXiv: 2002.08384] [INSPIRE].

[117] COMPASS collaboration, First Measurement of the Transverse Spin Asymmetries of the Deuteron in Semi-inclusive Deep Inelastic Scattering, Phys. Rev. Lett. 94 (2005) 202002 [hep-ex/0503002] [INSPIRE].

[118] COMPASS collaboration, A New measurement of the Collins and Sivers asymmetries on a transversely polarised deuteron target, Nucl. Phys. B 765 (2007) 31 [hep-ex/0610068] [INSPIRE].

[119] COMPASS collaboration, I - Experimental investigation of transverse spin asymmetries in

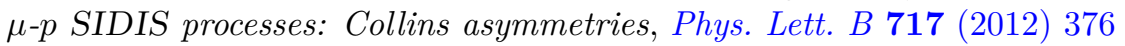
[arXiv: 1205.5121] [INSPIRE].

[120] COMPASS collaboration, Collins and Sivers asymmetries for pions and kaons in muon-deuteron DIS, Phys. Lett. B 673 (2009) 127 [arXiv:0802.2160] [INSPIRE].

[121] COMPASS collaboration, Collins and Sivers asymmetries in muonproduction of pions and kaons off transversely polarised protons, Phys. Lett. B 744 (2015) 250 [arXiv:1408.4405] [INSPIRE].

[122] Jefferson Lab Hall A collaboration, Single Spin Asymmetries in Charged Pion Production from Semi-Inclusive Deep Inelastic Scattering on a Transversely Polarized ${ }^{3} \mathrm{He}$ Target, Phys. Rev. Lett. 107 (2011) 072003 [arXiv:1106. 0363] [INSPIRE].

[123] Belle collaboration, Measurement of Azimuthal Asymmetries in Inclusive Production of Hadron Pairs in $e^{+} e^{-}$Annihilation at Belle, Phys. Rev. Lett. 96 (2006) 232002 [hep-ex/0507063] [INSPIRE].

[124] BELLE collaboration, Measurement of azimuthal asymmetries in inclusive production of hadron pairs in $e^{+} e^{-}$annihilation at $\sqrt{s}=10.58 \mathrm{GeV}$, Phys. Rev. D 78 (2008) 032011 [Erratum ibid. 86 (2012) 039905] [arXiv:0805.2975] [INSPIRE].

[125] BABAR collaboration, Measurement of Collins asymmetries in inclusive production of charged pion pairs in $e^{+} e^{-}$annihilation at BABAR, Phys. Rev. D 90 (2014) 052003 [arXiv: 1309.5278] [INSPIRE]. 
[126] BESIII collaboration, Measurement of Azimuthal Asymmetries in Inclusive Charged Dipion Production in $e^{+} e^{-}$Annihilations at $\sqrt{s}=3.65 \mathrm{GeV}$, Phys. Rev. Lett. 116 (2016) 042001 [arXiv: 1507.06824] [INSPIRE].

[127] HERMES collaboration, Evidence for a transverse single-spin asymmetry in leptoproduction of $\pi^{+} \pi^{-}$pairs, JHEP 06 (2008) 017 [arXiv:0803.2367] [INSPIRE].

[128] COMPASS collaboration, Transverse spin effects in hadron-pair production from semi-inclusive deep inelastic scattering, Phys. Lett. B 713 (2012) 10 [arXiv:1202.6150] [INSPIRE].

[129] COMPASS collaboration, A high-statistics measurement of transverse spin effects in dihadron production from muon-proton semi-inclusive deep-inelastic scattering, Phys. Lett. $B 736$ (2014) 124 [arXiv:1401.7873] [INSPIRE].

[130] Belle collaboration, Observation of Transverse Polarization Asymmetries of Charged Pion Pairs in $e^{+} e^{-}$Annihilation near $\sqrt{s}=10.58 \mathrm{GeV}$, Phys. Rev. Lett. 107 (2011) 072004 [arXiv:1104.2425] [INSPIRE].

[131] STAR collaboration, Observation of Transverse Spin-Dependent Azimuthal Correlations of Charged Pion Pairs in $p^{\uparrow}+p$ at $\sqrt{s}=200$ GeV, Phys. Rev. Lett. 115 (2015) 242501 [arXiv: 1504.00415] [INSPIRE].

[132] A. Bacchetta, A. Courtoy and M. Radici, First extraction of valence transversities in a collinear framework, JHEP 03 (2013) 119 [arXiv:1212.3568] [INSPIRE].

[133] M. Radici, A. Courtoy, A. Bacchetta and M. Guagnelli, Improved extraction of valence transversity distributions from inclusive dihadron production, JHEP 05 (2015) 123 [arXiv: 1503.03495] [INSPIRE].

[134] M. Radici and A. Bacchetta, First Extraction of Transversity from a Global Analysis of Electron-Proton and Proton-Proton Data, Phys. Rev. Lett. 120 (2018) 192001 [arXiv: 1802.05212] [INSPIRE].

[135] J. Benel, A. Courtoy and R. Ferro-Hernandez, A constrained fit of the valence transversity distributions from dihadron production, Eur. Phys. J. C 80 (2020) 465 [arXiv:1912.03289] [INSPIRE].

[136] JLQCD collaboration, Nucleon charges with dynamical overlap fermions, Phys. Rev. D 98 (2018) 054516 [arXiv: 1805.10507] [inSPIRE].

[137] R. Gupta, Y.-C. Jang, B. Yoon, H.-W. Lin, V. Cirigliano and T. Bhattacharya, Isovector charges of the nucleon from 2+1+1-flavor lattice QCD, Phys. Rev. D 98 (2018) 034503 [arXiv: 1806. 09006] [INSPIRE].

[138] C. Alexandrou, K. Cichy, M. Constantinou, K. Jansen, A. Scapellato and F. Steffens, Transversity parton distribution functions from lattice QCD, Phys. Rev. D 98 (2018) 091503 [arXiv: 1807.00232] [INSPIRE].

[139] BABAR collaboration, Collins asymmetries in inclusive charged $K K$ and $K \pi$ pairs produced in $e^{+} e^{-}$annihilation, Phys. Rev. D 92 (2015) 111101 [arXiv:1506.05864] [INSPIRE].

[140] M. Anselmino et al., Extracting the kaon Collins function from $e^{+} e^{-}$hadron pair production data, Phys. Rev. D 93 (2016) 034025 [arXiv: 1512.02252] [INSPIRE].

[141] A.V. Efremov, K. Goeke and P. Schweitzer, Predictions for azimuthal asymmetries in pion and kaon production in SIDIS off a longitudinally polarized deuterium target at HERMES, Eur. Phys. J. C 24 (2002) 407 [hep-ph/0112166] [INSPIRE].

[142] JefFerson Lab HaLl A collaboration, Single spin asymmetries in charged kaon production from semi-inclusive deep inelastic scattering on a transversely polarized ${ }^{3} \mathrm{He}$ target, Phys. Rev. C 90 (2014) 055201 [arXiv:1404.7204] [INSPIRE]. 
[143] X. Artru, J. Czyżewski and H. Yabuki, Single spin asymmetry in inclusive pion production, Collins effect and the string model, Z. Phys. C 73 (1997) 527 [hep-ph/9508239] [InSPIRE].

[144] B. Andersson, G. Gustafson, G. Ingelman and T. Sjöstrand, Parton fragmentation and string dynamics, Phys. Rept. 97 (1983) 31 [INSPIRE].

[145] X. Wang, Y. Yang and Z. Lu, Double Collins effect in $e^{+} e^{-} \rightarrow \Lambda \bar{\Lambda} X$ and $e^{+} e^{-} \rightarrow \Lambda \pi X$ processes in a diquark spectator model, Phys. Rev. D 97 (2018) 114015 [arXiv:1802.01843] [INSPIRE].

[146] A.V. Efremov, K. Goeke, S. Menzel, A. Metz and P. Schweitzer, Sivers effect in semi-inclusive DIS and in the Drell-Yan process, Phys. Lett. B 612 (2005) 233 [hep-ph/0412353] [INSPIRE].

[147] W. Vogelsang and F. Yuan, Single-transverse spin asymmetries: From deep inelastic scattering to hadronic collisions, Phys. Rev. D 72 (2005) 054028 [hep-ph/0507266] [INSPIRE].

[148] M. Anselmino, M. Boglione, U. D’Alesio, A. Kotzinian, F. Murgia and A. Prokudin, Extracting the Sivers function from polarized semi-inclusive deep inelastic scattering data and making predictions, Phys. Rev. D 72 (2005) 094007 [Erratum ibid. 72 (2005) 099903] [hep-ph/0507181] [INSPIRE].

[149] M. Anselmino, M. Boglione, U. D'Alesio, A. Kotzinian, F. Murgia and A. Prokudin, Role of Cahn and Sivers effects in deep inelastic scattering, Phys. Rev. D 71 (2005) 074006 [hep-ph/0501196] [INSPIRE].

[150] J.C. Collins, A.V. Efremov, K. Goeke, S. Menzel, A. Metz and P. Schweitzer, Sivers effect in semiinclusive deeply inelastic scattering, Phys. Rev. D 73 (2006) 014021 [hep-ph/0509076] [INSPIRE].

[151] M. Anselmino et al., Sivers effect for pion and kaon production in semi-inclusive deep inelastic scattering, Eur. Phys. J. A 39 (2009) 89 [arXiv:0805.2677] [InSPIRE].

[152] M. Anselmino, M. Boglione and S. Melis, Strategy towards the extraction of the Sivers function with transverse momentum dependent evolution, Phys. Rev. D 86 (2012) 014028 [arXiv: 1204.1239] [INSPIRE].

[153] P. Sun and F. Yuan, Energy evolution for the Sivers asymmetries in hard processes, Phys. Rev. D 88 (2013) 034016 [arXiv:1304.5037] [InSPIRE].

[154] L. Gamberg, Z.-B. Kang and A. Prokudin, Indication on the Process Dependence of the Sivers Effect, Phys. Rev. Lett. 110 (2013) 232301 [arXiv:1302.3218] [INSPIRE].

[155] M.G. Echevarria, A. Idilbi, Z.-B. Kang and I. Vitev, QCD evolution of the Sivers asymmetry, Phys. Rev. D 89 (2014) 074013 [arXiv:1401.5078] [InSPIRE].

[156] M. Anselmino, M. Boglione, U. D'Alesio, F. Murgia and A. Prokudin, Study of the sign change of the Sivers function from STAR Collaboration $W / Z$ production data, JHEP 04 (2017) 046 [arXiv: 1612.06413] [INSPIRE].

[157] M. Boglione, U. D'Alesio, C. Flore and J.O. Gonzalez-Hernandez, Assessing signals of TMD physics in SIDIS azimuthal asymmetries and in the extraction of the Sivers function, JHEP 07 (2018) 148 [arXiv:1806.10645] [INSPIRE].

[158] COMPASS collaboration, II - Experimental investigation of transverse spin asymmetries in $\mu$-p SIDIS processes: Sivers asymmetries, Phys. Lett. B 717 (2012) 383 [arXiv: 1205.5122] [INSPIRE].

[159] M. Burkardt, Quark correlations and single spin asymmetries, Phys. Rev. D 69 (2004) 057501 [hep-ph/0311013] [INSPIRE]. 
[160] M. Burkardt, Sivers mechanism for gluons, Phys. Rev. D 69 (2004) 091501 [hep-ph/0402014] [INSPIRE].

[161] A. Signori, A. Bacchetta, M. Radici and G. Schnell, Investigations into the flavor dependence of partonic transverse momentum, JHEP 11 (2013) 194 [arXiv:1309.3507] [INSPIRE].

[162] HERMES collaboration, Quark helicity distributions in the nucleon for up, down, and strange quarks from semi-inclusive deep-inelastic scattering, Phys. Rev. D 71 (2005) 012003 [hep-ex/0407032] [INSPIRE].

[163] D. de Florian, R. Sassot and M. Stratmann, Global analysis of fragmentation functions for protons and charged hadrons, Phys. Rev. D 76 (2007) 074033 [arXiv:0707.1506] [INSPIRE].

[164] M. Echevarria and G. Schnell, Sivers asymmetries for electroproduction of protons and antiprotons, to be published.

[165] C. Lefky and A. Prokudin, Extraction of the distribution function $h_{1 T}^{\perp}$ from experimental data, Phys. Rev. D 91 (2015) 034010 [arXiv: 1411.0580] [InSPIRE].

[166] A. Kotzinian, B. Parsamyan and A. Prokudin, Predictions for double spin asymmetry $A_{L T}$ in semiinclusive DIS, Phys. Rev. D 73 (2006) 114017 [hep-ph/0603194] [INSPIRE].

[167] B.U. Musch, P. Hägler, J.W. Negele and A. Schäfer, Exploring quark transverse momentum distributions with lattice QCD, Phys. Rev. D 83 (2011) 094507 [arXiv:1011.1213] [INSPIRE].

[168] B. Yoon et al., Nucleon transverse momentum-dependent parton distributions in lattice QCD: Renormalization patterns and discretization effects, Phys. Rev. D 96 (2017) 094508 [arXiv:1706.03406] [INSPIRE].

[169] W. Mao, Z. Lu and B.-Q. Ma, Transverse single-spin asymmetries of pion production in semi-inclusive DIS at subleading twist, Phys. Rev. D 90 (2014) 014048 [arXiv:1405.3876] [INSPIRE].

[170] B. Parsamyan, Measurement of longitudinal-target-polarization dependent azimuthal asymmetries in SIDIS at COMPASS experiment, PoS DIS2017 (2018) 259 [arXiv: 1801.01488] [INSPIRE].

[171] CLAS collaboration, Measurement of Single- and Double-Spin Asymmetries in Deep Inelastic Pion Electroproduction with a Longitudinally Polarized Target, Phys. Rev. Lett. 105 (2010) 262002 [arXiv: 1003.4549] [INSPIRE].

[172] HERMES collaboration, Single-spin azimuthal asymmetry in exclusive electroproduction of $\pi^{+}$mesons on transversely polarized protons, Phys. Lett. B 682 (2010) 345 [arXiv: 0907.2596] [INSPIRE].

[173] K. Kanazawa, Y. Koike, A. Metz and D. Pitonyak, Towards an explanation of transverse single-spin asymmetries in proton-proton collisions: The role of fragmentation in collinear factorization, Phys. Rev. D 89 (2014) 111501 [arXiv:1404.1033] [InSPIRE].

[174] L. Gamberg, Z.-B. Kang, D. Pitonyak and A. Prokudin, Phenomenological constraints on $A_{N}$ in $p^{\uparrow} p \rightarrow \pi X$ from Lorentz invariance relations, Phys. Lett. B $\mathbf{7 7 0}$ (2017) 242 [arXiv: 1701.09170] [INSPIRE].

[175] HERMES collaboration, Longitudinal double-spin asymmetries in semi-inclusive deep-inelastic scattering of electrons and positrons by protons and deuterons, Phys. Rev. D 99 (2019) 112001 [arXiv:1810.07054] [INSPIRE].

[176] HERMES collaboration, Single-spin azimuthal asymmetry in exclusive electroproduction of $\pi^{+}$mesons, Phys. Lett. B 535 (2002) 85 [hep-ex/0112022] [INSPIRE]. 
[177] K.A. Oganessyan, P.J. Mulders and E. De Sanctis, Double-spin $\cos \phi$ asymmetry in semi-inclusive electroproduction, Phys. Lett. B 532 (2002) 87 [hep-ph/0201061] [INSPIRE].

[178] M. Anselmino, A. Efremov, A. Kotzinian and B. Parsamyan, Transverse momentum dependence of the quark helicity distributions and the Cahn effect in double-spin asymmetry $A_{L L}$ in semiinclusive DIS, Phys. Rev. D 74 (2006) 074015 [hep-ph/0608048] [INSPIRE].

[179] COMPASS collaboration, Azimuthal asymmetries of charged hadrons produced by high-energy muons scattered off longitudinally polarised deuterons, Eur. Phys. J. C 70 (2010) 39 [arXiv: 1007.1562] [INSPIRE].

[180] L. Trentadue and G. Veneziano, Fracture functions. An improved description of inclusive hard processes in QCD, Phys. Lett. B 323 (1994) 201 [INSPIRE].

[181] M. Anselmino, V. Barone and A. Kotzinian, SIDIS in the target fragmentation region: Polarized and transverse momentum dependent fracture functions, Phys. Lett. B 699 (2011) 108 [arXiv: 1102.4214] [INSPIRE].

[182] A. Bacchetta, D. Boer, M. Diehl and P.J. Mulders, Matches and mismatches in the descriptions of semi-inclusive processes at low and high transverse momentum, JHEP 08 (2008) 023 [arXiv: 0803.0227] [INSPIRE].

[183] L.L. Pappalardo, Transverse spin effects in polarized semi inclusive deep inelastic scattering, Ph.D. thesis, University of Ferrara, Italy (2008).

[184] M. Diefenthaler, Signals for transversity and transverse momentum dependent quark distribution functions studied at the HERMES experiment, Ph.D. thesis, University Erlangen-Nuremberg, Germany (2010).

[185] F. James and M. Roos, Minuit - a system for function minimization and analysis of the parameter errors and correlations, Comput. Phys. Commun. 10 (1975) 343 [INSPIRE]. 